\title{
Asymmetric Ring-Opening of Epoxides Catalyzed by Metal-Salen Complexes
}

\author{
Anna Lidskog ${ }^{+}\left(\mathbb{0}\right.$, Yutang $\mathrm{Li}^{\dagger}$ and Kenneth Wärnmark *(i) \\ Centre for Analysis and Synthesis, Lund University, P.O Box 124, SE-22100 Lund, Sweden; \\ Anna.Lidskog@chem.lu.se (A.L.); Yutang.Li@chem.lu.se (Y.L.) \\ * Correspondence: kenneth.warnmark@chem.lu.se; Tel.: +46-46-222-8217 \\ + These authors equally contributed to this work.
}

Received: 4 June 2020; Accepted: 18 June 2020; Published: 23 June 2020

\begin{abstract}
The asymmetric ring-opening of epoxides is an important reaction in organic synthesis, since it allows for the enantioselective installation of two vicinal functional groups with specific stereochemistry within one step from a highly available starting material. An effective class of catalysts for the asymmetric ring-opening of epoxides is metal-salen complexes. This review summarizes the development of metal-salen catalyzed enantioselective desymmetrization of meso-epoxides and kinetic resolution of epoxides with various nucleophiles, including the design and application of both homogeneous- and heterogeneous epoxide-opening catalysts as well as multi-metallic covalent and supramolecular catalytic systems.
\end{abstract}

Keywords: asymmetric catalysis; salen complexes; ring-opening; epoxide; kinetic resolution

\section{Introduction}

The availability of enantiomerically pure or enriched compounds is crucial for several different fields of chemistry including pharmaceutical, biological, agricultural, and materials chemistry [1,2]. The high demand for chiral compounds has inspired extensive research into the development of practical and efficient methods for their preparation. One of the main tools for obtaining enantiomerically pure compounds is asymmetric catalysis. In the field of asymmetric catalysis, enantiomerically pure catalysts are employed to transform prochiral or racemic substrates into valuable enantioenriched compounds.

Chiral metal-salen complexes are privileged catalysts, meaning that they are demonstrating enantioselectivity over a wide range of reactions and substrates [3,4]. Salen ligands have attracted much attention due to the fact of their tunable steric and electronic properties as well as their ability to coordinate a large number of different metal ions and stabilize them in various oxidation states $[5,6]$. In addition, the commercial availability of starting materials, such as enantiomerically pure vicinal diamines, and well-established synthetic procedures allows for the facile preparation of a wide variety of enantiomerically pure salen ligands and their corresponding metal complexes in high yields (Scheme 1).
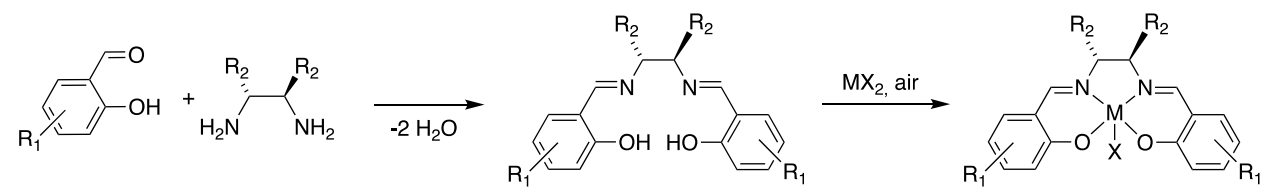

Scheme 1. The synthesis of enantiomerically pure salen ligands and corresponding metal-salen complexes. The ligand synthesis is a Schiff base reaction. The formation of the metal-salen complex is illustrated as the insertion of the metal in oxidation state $+\mathrm{II}$ followed by oxidation in air to oxidation state + III. 
Epoxides represent an important class of compounds in organic synthesis due to the fact of their high availability and facile, stereoselective, and often regioselective nucleophilic ring-opening, leading to multifunctional organic compounds in very few steps [7]. Achiral and racemic epoxides can be easily prepared by the oxidation of simple alkene precursors, and much effort has also been focused on the development of enantioselective epoxidations [8].

The asymmetric ring-opening (ARO) of epoxides affords enantiopure vicinal difunctionalized organic compounds with two adjacent stereocenters [9,10]. The relative stereochemistry of two stereocenters in the ring-opened product depends on the configuration of the epoxide (Scheme 2). The use of chiral Lewis acids, such as metal-salen complexes, has been shown to significantly increase the reactivity and enantioselectivity of ARO reactions. An alternative way of obtaining the same product would be to first perform an enantioselective epoxidation, followed by selective ring-opening of the enantiomerically pure epoxide.

(a)<smiles>[X]C1CC2CC1O2</smiles>

meso

(b)<smiles>[R]C1OC1[R7]</smiles>

$\mathrm{R}_{1}=\mathrm{R}_{2}$ meso

$\mathrm{R}_{1} \neq \mathrm{R}_{2}$ cis
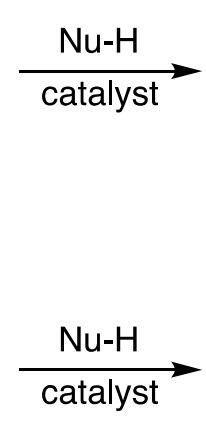<smiles>[R2]C([NH3+])C([R])O</smiles><smiles>C#CC</smiles><smiles>[R]C(N)C([R])O</smiles>

syn

(c)<smiles></smiles><smiles>C[13CH][13CH]</smiles>

trans

(d)<smiles>[R]C1CO1</smiles>

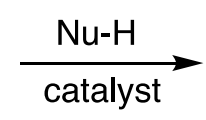

Scheme 2. The asymmetric ring-opening of epoxides and the resulting relative stereochemistry of the ring-opened products. Ring-opening of (a) cyclic meso-epoxides to yield a ring-opened product with trans-stereochemistry; (b) acyclic meso-epoxides and cis-epoxides to yield ring-opened product with syn-stereochemistry; (c) trans-epoxides to yield ring-opened product with anti-stereochemistry; (d) terminal epoxides.

There are two main types of epoxides that have been used in ARO reactions: achiral (meso) epoxides and racemic epoxides. In the former case, the desymmetrization of meso-epoxides with a suitable optically pure catalyst leads to the formation of chiral vicinally substituted alcohols with trans- (for cyclic meso-epoxides) and syn stereochemistry (for acyclic meso-epoxides) in up to 100\% yield and enantiomeric excess. In the latter case, the catalyst has to be able to differentiate between the two enantiomers and preferentially transform one of them into the ring-opened product with high regioselectivity and enantioselectivity. This process is a kinetic resolution (KR). While the 
maximum yield of the ring-opened product is only $50 \%$, this method also allows for the simultaneous enantioenrichment of the unreacted epoxide, enabling, for example, the preparation of optically enriched terminal epoxides.

In this review, we present an overview of the development and application of metal-salen complexes as catalysts for the ARO of epoxides. Previous reviews in this area have either been focused on the ARO of epoxides in general and covered a number of different salen and non-salen catalysts $[9,10]$ or focused on the use of metal-salen complexes as catalysts for several different reactions [4]. There are also a number of reviews focused on multi-metallic salen complexes [11-13] and heterogeneous salen complexes $[14,15]$ but, again, covering several different types of reactions. As several of the mentioned reviews were published 5-15 years ago, we here provide an up-to-date and comprehensive overview of the field, covering the literature until the beginning of 2020 .

\section{Desymmetrization of meso-Epoxides}

\subsection{With Azides}

The enantioselective ring-opening of epoxides with azides is a very important reaction due to the facile conversion of the products into valuable vicinal amino alcohols of very high optical purity (Scheme 3) [16-19]. The first report of a metal-salen catalyst used for an ARO reaction came from Jacobsen and coworkers [20] in 1995 with the use of $\mathrm{Cr}(\mathrm{III})$ salen complex $\mathbf{1}$ for the ARO of epoxides with trimethylsilylazide $\left(\mathrm{TMSN}_{3}\right.$ ) as nucleophile (Scheme 4$)$. Since then, considerable efforts have been invested into improving this catalytic system, including developing multi-metallic catalysts and employing different heterogenization strategies. The $\mathrm{Cr}(\mathrm{III})$ salen complexes remain the most investigated catalysts for the ARO of epoxides with azides as nucleophile, and only a few less efficient salen complexes based on other metals can be found in the literature [21-23].

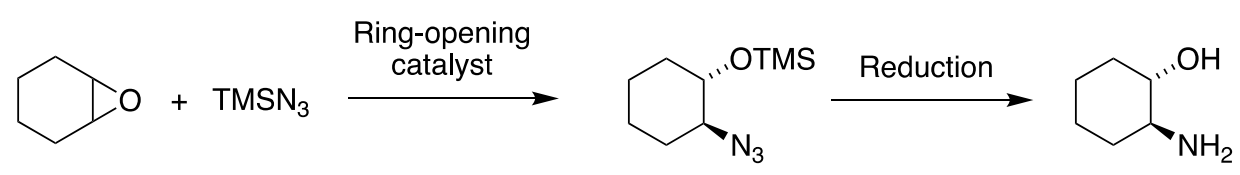

Scheme 3. The asymmetric ring-opening of cyclohexene oxide with $\mathrm{TMSN}_{3}$ followed by reduction to synthesize the corresponding trans-1,2-amino alcohol with high enantioselectivity.
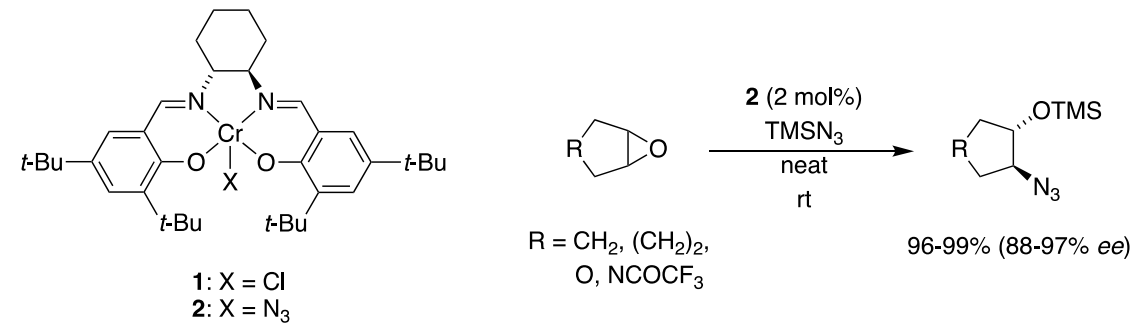

Scheme 4. The asymmetric azidolysis of meso-epoxides catalyzed by $\mathrm{Cr}$-salen complexes.

The seminal work of Jacobsen and coworkers on chiral Cr-salen complexes for the asymmetric catalysis of the azidolysis of meso-epoxides have yielded invaluable insight in the development of catalysts for the ARO of epoxides. Drawing on knowledge gained in their previous work on the asymmetric epoxidation of alkenes [24-26], they found that by using the same salen ligand and exchanging the manganese(III) for chromium(III) (complex 1), the ARO of cyclic meso-epoxides with $\mathrm{TMSN}_{3}$ as nucleophile could be catalyzed in high yields and with high enantioselectivities (up to 99\% yield and $97 \% e e$, Scheme 4). The reactions could be conducted either in ethereal solution or under solvent-free conditions with similar yields and enantioselectivities [20,27].

The stability of the catalyst under the reaction conditions facilitated the mechanistic studies, which showed that the active catalyst was the $\mathrm{Cr}$-salen azide complex 2 (Scheme 4 ). This complex could 
be isolated from the reaction mixture and recycled up to 10 times at $1 \mathrm{~mol} \%$ loading with maintained reactivity and enantioselectivity. Kinetic studies revealed a second-order dependence of the reaction rate on the catalyst, suggesting that the catalyst played a dual role in the mechanism: activating both the electrophile and the nucleophile in a bimetallic rate-determining step (Scheme 5) [28]. The proposed mechanism was also supported by the observation of significant non-linear effects of the enantiomeric composition of the catalyst on the enantioselectivity of the reaction [29]. Studies also showed that $\mathrm{TMSN}_{3}$ was not directly involved in the catalytic cycle but rather served as a source of $\mathrm{HN}_{3}$ in the presence of trace amounts of water [28].

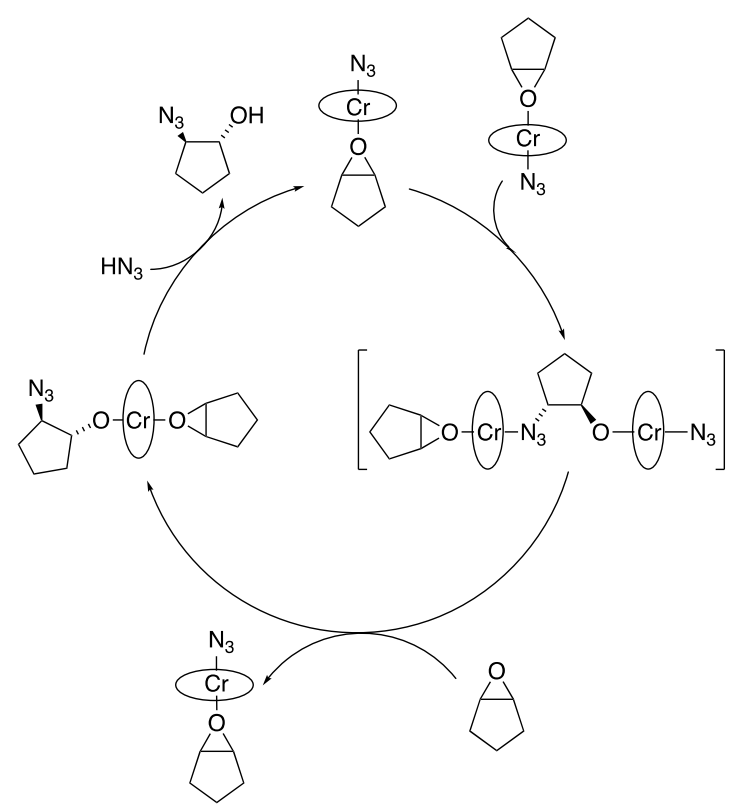

Scheme 5. The mechanism for the ARO of cyclopentene oxide with $\mathrm{TMSN}_{3}$ catalyzed by Cr-salen complexes as proposed by Jacobsen et al. [28].

The proposal of a bimetallic rate-determining step led to the synthesis and application of dimeric catalysts, both in order to gain further mechanistic insights and in the hope that enforcing the cooperativity would lead to improved catalytic activity and enantioselectivity. Jacobsen and coworkers [30] designed and synthesized a number of covalently linked dimeric complexes with different position and length of the linker (Figure 1), which were evaluated as catalysts for the solvent-free ARO of cyclopentene oxide with $\mathrm{TMSN}_{3}$. The positioning of the linker was based on two limiting geometries envisioned for the transition state of the ARO of meso-epoxides catalyzed by monomeric complex 2: "head-to-head" and "head-to-tail" (Figure 2). Complex 3 was designed to evaluate the "head-to-head" geometry and complexes 4-10 were designed to evaluate the "head-to-tail" geometry.
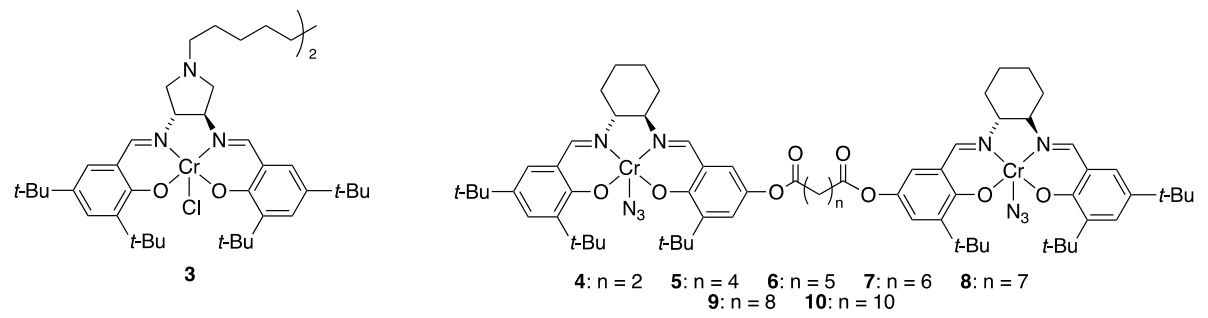

Figure 1. Bimetallic catalysts designed to evaluate the "head-to-head" geometry (catalyst 3) and the "head-to-tail" geometry (catalyst 4-10) of the bimolecular catalysis. 

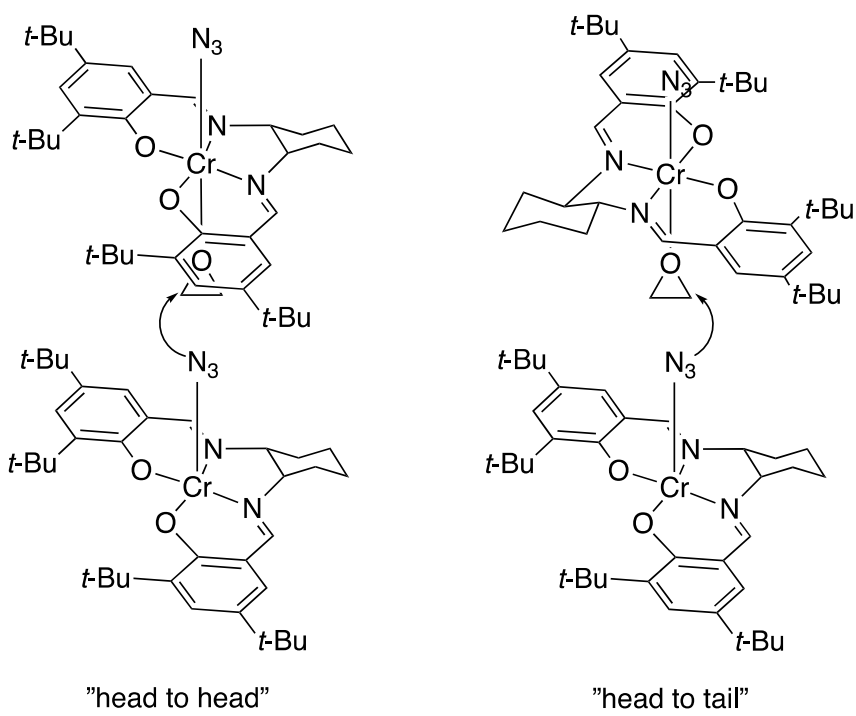

Figure 2. The two limiting geometries for the enantioselectivity-determining transition state of the asymmetric ring-opening of meso-epoxides catalyzed by $\mathrm{Cr}$-salen complex 2 as proposed by Jacobsen et al. [30].

All of the investigated dimeric complexes could be used under solvent-free conditions and gave increased reaction rates in the ARO of cyclopentene oxide with $\mathrm{TMSN}_{3}$ compared to monomeric analogues. While the complex based on the "head-to-head" alignment (complex 3) gave very low enantioselectivity ( $8 \%$ ee), the "head-to-tail" aligned complexes $\mathbf{4 - 1 0}$ gave enantioselectivities similar to those obtained with monomeric analogues (90-94\% ee). Complexes $\mathbf{4 - 1 0}$ also performed efficient catalysis at concentrations one order of magnitude below the lower limit of reactivity for the monomeric analogues. Kinetic studies showed the participation of both inter- and intramolecular pathways in the ARO, with complex $6(n=5)$ demonstrating the highest rate constants for both the inter- and intramolecular pathways out of the investigated dimeric catalysts (4-10). The intramolecular cooperative catalysis could also be observed by the decreasing non-linear effects in the ARO of cyclopentene oxide with $\mathrm{TMSN}_{3}$ with decreasing concentration of the dimeric catalyst $[29,30]$.

Inspired by Jacobsen's dimeric Cr-salen complexes (Figure 1), Wärnmark and coworkers designed and synthesized several heterobimetallic dimeric complexes, based on the idea that some metal-salen complexes might be more efficient at activating the epoxide and others might be better at activating the nucleophile. This was supported by the initial screening of different 1:1 combinations of monometallic salen complexes with different metal ions as catalysts in the ARO of cyclohexene oxide with $\mathrm{TMSN}_{3}$, where synergistic effects in terms of reactivity and enantioselectivity were observed when $\mathrm{Cr}$-monosalen complexes were combined with $\mathrm{Mn}-$ or $\mathrm{Co}-$ monosalen complexes. The design of the dimeric complexes included a novel design principle, where $C_{2}$ - and $C_{\mathrm{s}}$-symmetric ligands are desymmetrized by the insertion of two different metal ions, giving catalysts of pseudo- $C_{2}$ [31] and pseudo- $C_{\mathrm{s}}$ [32] symmetry, respectively (Figure 3). The catalysts were applied in the ARO of a number of meso-epoxides with $\mathrm{TMSN}_{3}$. The pseudo- $C_{2}$ catalysts, $\mathrm{Cr}(\mathrm{III})-\mathrm{Co}(\mathrm{III})$ catalyst 12 gave the best results; the reaction could be performed under solvent-free conditions and with very low catalyst loading $(0.01 \mathrm{~mol} \%)$. The ring-opened products were obtained in excellent yields and enantioselectivities (up to $99 \%$ yield and 94\% ee). The $\mathrm{Cr}(\mathrm{III})-\mathrm{Co}(\mathrm{III})$ catalyst 12 (Figure 3) exhibited higher enantioselectivity than monomeric analogue 1 (Scheme 4) and dimeric homobimetallic complex $\mathbf{6}$ (Figure 1) in the ARO of cyclohexene oxide under the same reaction conditions. $\mathrm{Cr}$ (III)-Mn(III) catalyst 11 (Figure 3) displayed the highest reactivity, with a turnover frequency (TOF) fifty times higher than monomeric $\mathbf{1}$ and five times higher than homometallic complex 6 (Figure 1) under the same reaction conditions. The study also included a number of dimeric catalysts with different diamine backbones, although the best results were obtained for the catalysts shown in Figure 3 with trans-1,2-diaminocyclohexyl backbones. For the pseudo- $C_{\mathrm{s}}$ 
catalysts 13 and 14 (Figure 3), the ligand itself is achiral, and the chirality is induced only by the coordination of two different metal ions. Even so, this weak source of chirality was enough to induce enantioselectivity and the complexes were found to catalyze the ARO of cyclopentene oxide with $\mathrm{TMSN}_{3}$ under solvent-free conditions, giving the ring-opened product in $>99 \%$ yield and $63-76 \% e e$.
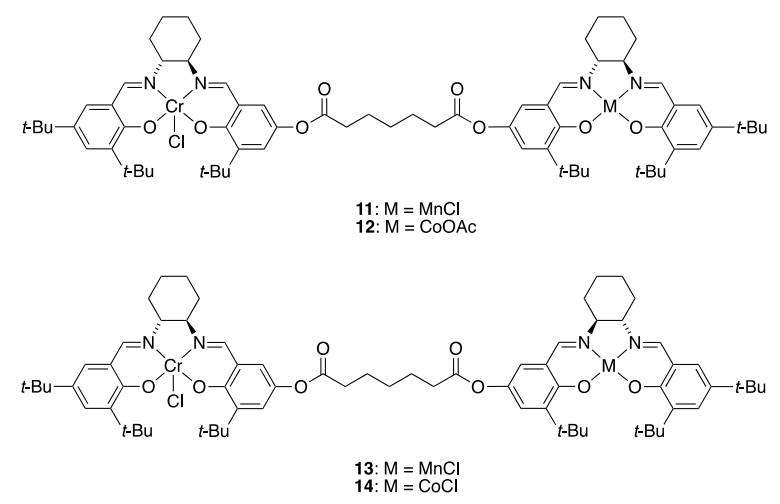

Figure 3. Pseudo- $C_{2}$ and pseudo- $C_{\mathrm{s}}$-symmetric heterobimetallic bissalen complexes.

Another way of exploiting the cooperative mechanism is by designing catalysts where metal-salen complexes are brought into close proximity by non-covalent interactions. Hence, Mirkin and coworkers [33] developed an allosteric supramolecular catalyst by synthesizing a $\mathrm{Cr}$-salen complex with a 2-diphenylphosphanylethylsulfanyl linker at each end of the salen ligand. The dimeric allosteric catalyst was formed by connecting two $\mathrm{Cr}$-salen complexes through coordination to $\mathrm{Rh}(\mathrm{I})$ at each end (Figure 4). The distance between the two $\mathrm{Cr}$-salen units (changing between the closed and open forms) could be allosterically controlled by the reversible binding of a $\mathrm{CO}$ molecule and a chloride ion to each $\mathrm{Rh}(\mathrm{I})$ center (Figure 4). The catalytic properties were investigated in the ARO of cyclohexene oxide with $\mathrm{TMSN}_{3}$. Using the closed form of the catalyst (complex 15) gave $68 \%$ ee and a 20 -fold rate enhancement compared to the monomeric catalyst 1 under the same conditions. The open form of the catalyst (complex 16) generated a further doubling of the reaction rate. However, no ee values of the product were reported from the catalysis performed with the open form of the catalyst. Due to the poor solubility of the catalyst, all reactions were performed in benzonitrile.

The same research group also developed catalytically active molecular tweezers based on the same design principle with a single $\mathrm{Rh}(\mathrm{I})$ center acting as the hinge [34]. This complex allowed for reactions to be run in THF instead of benzonitrile. Using the catalyst in the ARO of cyclohexene oxide with $\mathrm{TMSN}_{3}$ yielded products with up to $80 \%$ ee, compared to $26 \%$ ee obtained with complex 1 under the same reaction conditions. The closed form of the catalyst maintained a high enantioselectivity over a range of concentrations, while the open (linear) form exhibited significantly decreased enantioselectivity at lower concentrations.

Wärnmark and coworkers $[35,36]$ explored hydrogen-bonded supramolecular catalysts, designing Cr-salen complexes capable of forming heterodimers through complementary hydrogen-bonding motifs (complexes 17 and 18 in Figure 5). In addition, complexes containing an alkyl strap were also synthesized (complexes 19 and $\mathbf{2 0}$ in Figure 5) in order to force the hydrogen-bonding moieties of the monomers into a favorable conformation to assist the aggregation of the desired cyclic heterodimer. The ARO of cyclohexene oxide and cyclopentene oxide with $\mathrm{TMSN}_{3}$ in toluene was used to evaluate the supramolecular systems. Kinetic studies showed that the supramolecular systems $\mathbf{1 7}+\mathbf{1 8}$ and $\mathbf{1 9}+$ 20, respectively, gave higher reaction rates than monosalen catalyst $\mathbf{1}$ (Scheme 4) with the strapped systems showing the highest catalytic efficacy. The effect was most pronounced at lower catalyst concentrations. As an example, at $5 \mathrm{mM}$ catalyst concentration in toluene, the strapped system $19+20$ gave eight times higher initial rate than monosalen catalyst 1 for the ARO of cyclohexene oxide. Studies also indicated that more than one reaction mechanism may be involved in the catalysis involving these systems. The induced enantioselectivity was, however, significantly lower $(<10 \% e e)$ than what 
was obtained with catalyst 1. Equilibrium studies on similar studies by the Wärnmark group [37] showed that only a fraction of the supramolecular assemblies contained the cyclic dimeric structure in solution. Hence, the catalysis was, instead, most likely conducted by a mixture of open, linearly aggregated species.
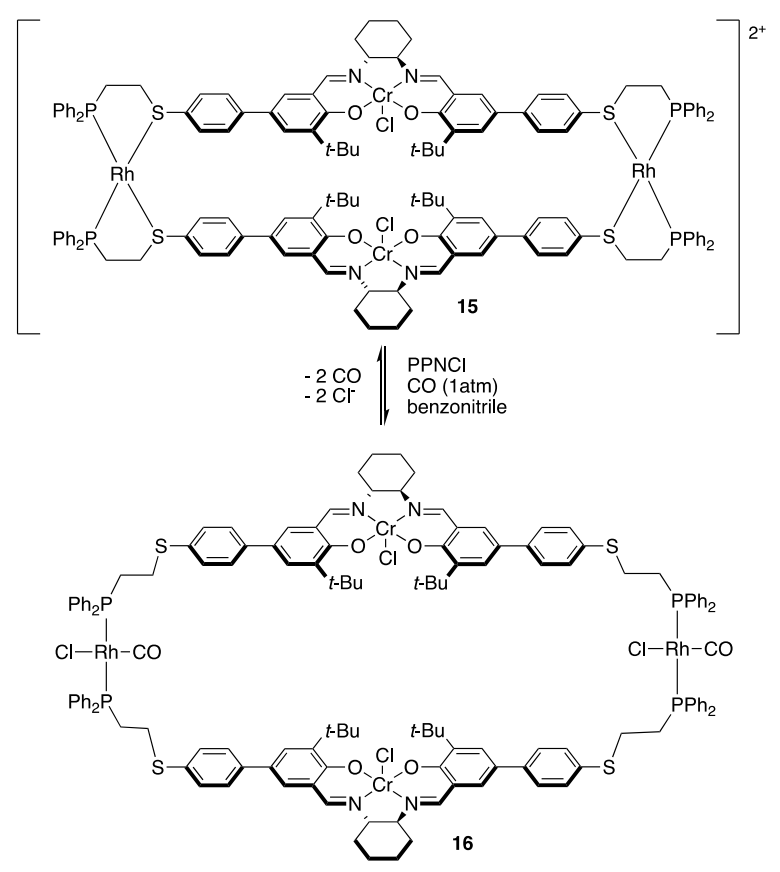

Figure 4. An allosteric supramolecular bissalen catalyst; closed form (top, 15) and open form (bottom, 16). $\mathrm{PPNCl}=$ bis(triphenylphosphoranylidene)ammonium chloride.
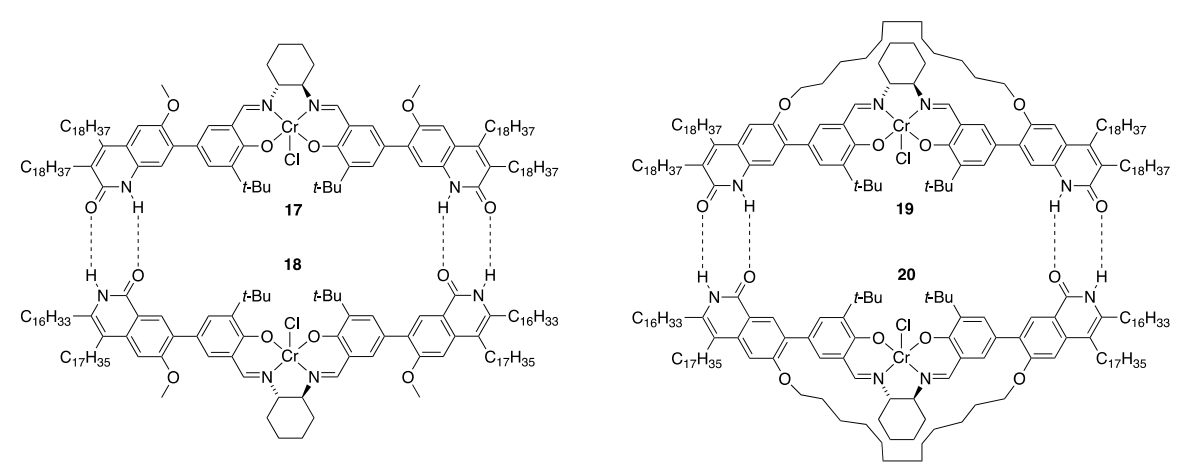

Figure 5. Monomeric catalysts 17-20, drawn as hydrogen-bonded cyclic dimeric aggregates.

One area of interest has been the development of heterogeneous metal-salen catalysts, as this potentially allows for an easier product separation and catalyst recovery $[14,15,38]$. Several different strategies have been explored for the immobilization of metal-salen complexes on different solid supporting materials [39]. In addition to the reactivity and enantioselectivity, the heterogeneous catalysts also need to be evaluated in terms of stability, leaching, and recyclability.

Early examples include polymer-supported chiral Cr-salen complexes [40] and cationic chiral Cr-salen complexes incorporated into the cavities of zeolites and the interlamellar region of montmorillonite [41]. Both of these strategies gave low enantioselectivities in the ARO of cyclic meso-epoxides with $\mathrm{TMSN}_{3}$. The decreased enantioselectivities compared to homogeneous catalysts were attributed to a changed steric environment around the complex $[40,41]$ and loss of the cooperative catalytic effect [41].

Garcia and co-workers [42] developed heterogeneous catalysts by binding chiral Cr-salen complexes to solid silicates. The complexes were anchored on functionalized silicates through 
aminopropyl tethers, either by complexation with the metal (complex 21, Figure 6) or by covalent linkage to the ligand (complex 22, Figure 6). The complexes anchored to the solid through coordination to the chromium catalyzed the ARO of cyclohexene oxide with $\mathrm{TMSN}_{3}$ with high yield (93-99\%) and up to $70 \%$ ee but were found to undergo extensive leaching of the complex into the diethyl ether solution. In contrast, the covalently linked complexes showed no leaching but generated only moderate yields and low enantiomeric excesses in the ring-opening of cyclohexene oxide (43-66\% yield and $8-18 \% e e$ ). The decreased enantioselectivity compared to homogeneous catalysts was attributed to a likely change in the reaction mechanism.
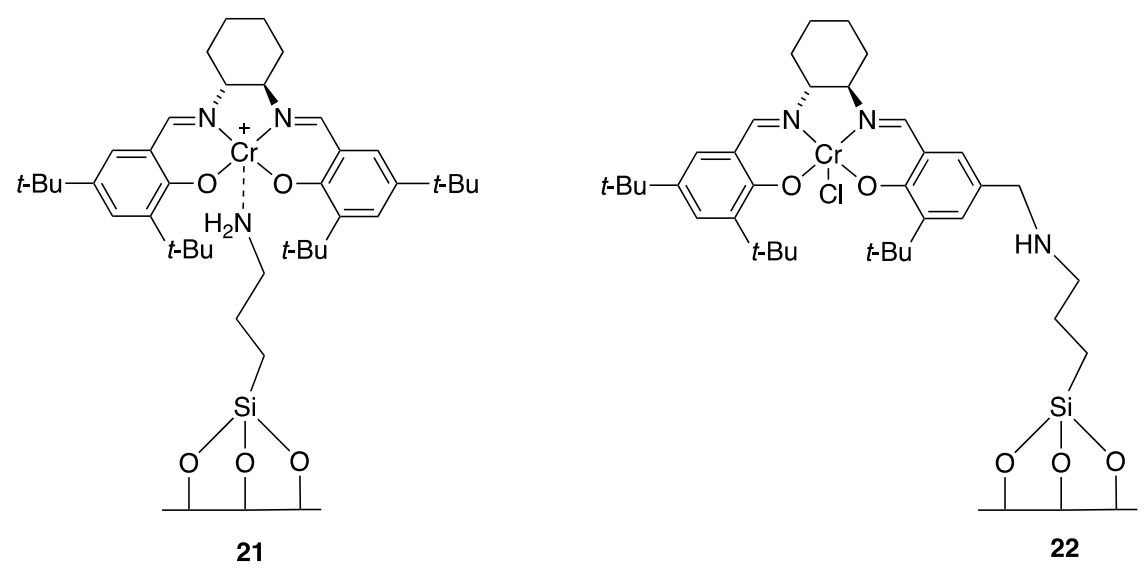

Figure 6. Cr-salen complexes anchored to functionalized silicates $\mathrm{SiO}_{2}$, ITQ-2 and MCM-41.

Jacobs and coworkers [43] conducted further investigations of heterogeneous catalyst 21 (Figure 6) in the ARO of meso-epoxides. They found that the level of $\mathrm{Cr}$-salen complex leaching is largely dependent on the choice of solvent. While homogeneously catalyzed ARO reactions of epoxides are usually performed in ethereal solvents, in heterogeneous catalysis, these solvents greatly enhanced the leaching by facilitating ligand exchange processes. Using apolar non-coordinating solvents like hexane gave leaching of $<1 \%$ in the ARO of cyclohexene oxide and cyclopentene oxide with $\mathrm{TMSN}_{3}$ as nucleophile, while maintaining excellent yields and good enantioselectivities (up to $77 \%$ ee). Recycling experiments showed that the catalyst could be recycled up to 10 times without loss of reactivity and enantioselectivity, although the reaction time had to be increased after each run.

Jacobs and coworkers also explored monomeric complex 1 (Scheme 4) and dimeric complex 23 (Figure 7) impregnated on unfunctionalized silica [44,45]. The heterogeneous catalysts were used in the ARO of cyclopentene oxide and cyclohexene oxide with $\mathrm{TMSN}_{3}$. All reactions were performed in hexane. The catalyst based on monomeric complexes exhibited moderate reactivity and enantioselectivity (47-98\% yield and 30-45\% ee), and while leaching was limited, the silica support suffered some deterioration after repeated experiment [45]. The catalyst based on dimeric complex 23 (Figure 7) gave higher yields (74-98\%) and improved, although still moderate, enantioselectivities $(50-68 \% e e)$. Using the catalyst in a continuous-flow reactor reduced the deterioration of the support, although these experiments were only performed for the KR of 1,2-epoxyhexane with $\mathrm{TMSN}_{3}$ [44]. In later studies, the dimeric $\mathrm{Cr}$-salen complex was also immobilized in a silica-supported ionic liquid, using ionic liquid [bmim] $\left[\mathrm{PF}_{6}\right]$ (bmim = 1-butyl-3-methylimidazolium) [46]. This type of heterogenization resulted in both increased reactivity and enantioselectivity compared to the dimeric catalyst impregnated on silica ( $93 \%$ yield and $75 \%$ ee in the ARO of cyclohexene oxide with $\mathrm{TMSN}_{3}$ ), as the catalyst in the supported ionic liquid phase was more accessible for reaction than when adsorbed on a silica surface. The catalyst and the ionic liquid could be recovered by Soxhlet extraction with acetone and recycled without loss of enantioselectivity. As reported for the catalysts impregnated on silica, the support shows some deterioration over time, but this could again be improved by using a continuous-flow reactor. 


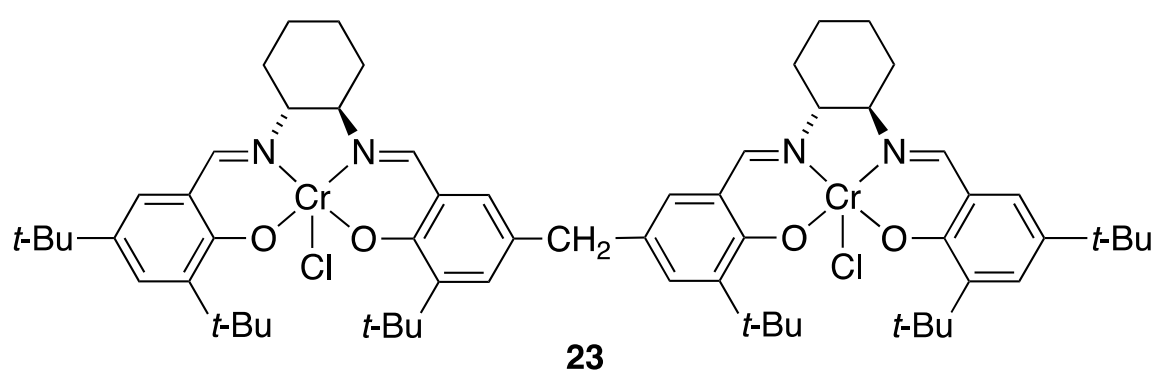

Figure 7. Dimeric Cr-salen complex 23.

Another approach to the heterogenization of $\mathrm{Cr}$-salen complexes is to use dendritic structures as a soluble support. Hence, Keilitz and Haag [47] developed catalysts based on Cr-salen complexes immobilized on hyperbranched polyglycerol (complexes 24-27, Figure 8). The catalysts consist of $\mathrm{Cr}$-salen analogues with a pyrrolidine backbone, which are linked to the support by linkers of different lengths. The catalysts were used in the ARO of cyclopentene oxide and cyclohexene oxide with $\mathrm{TMSN}_{3}$, using diethyl ether as solvent. The dendrimeric catalysts displayed significant rate enhancements compared to a monomeric analogue, but decreased enantioselectivity. By increasing the length of the linker, the enantioselectivity was improved (from 16\% to $64 \%$ ee for the ARO of cyclopentene oxide). The best results were obtained with a C10 linker (catalyst 26) which gave the ring-opened products with ee values of $48 \%$ (for the product from cyclohexene oxide) and $64 \%$ (for the product from cyclopentene oxide). Full conversions were reported for all reactions.

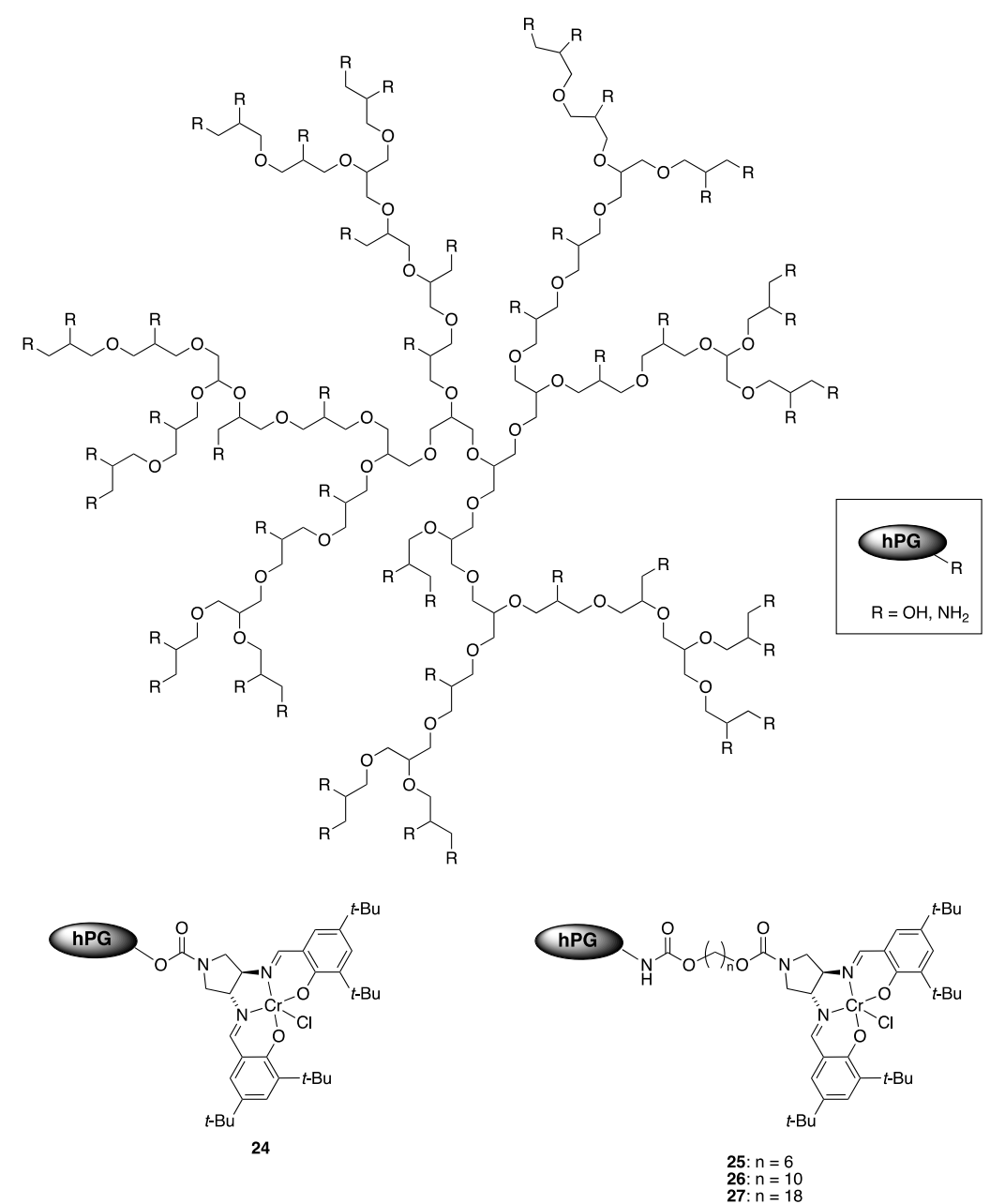

Figure 8. Cr-salen complexes immobilized on hyperbranched polyglycerol (hPG). 
Schulz and coworkers [38] synthesized a chiral calixsalen-type chromium complex that was employed as a heterogeneous catalyst in the ARO of cyclohexene oxide and 3,4-epoxytetrahydrofuran with $\mathrm{TMSN}_{3}$. The macrocyclic catalyst 28 contains $2-5$ repeating thiophene-salen units and was used as an oligomeric mixture (Figure 9). The reaction was performed under heterogeneous conditions in tert-butyl methyl ether (TBME), and the catalyst could be recovered by filtration and recycled several times. The products were obtained in good yields (62-90\%) and moderate enantioselectivities $(42-62 \% e e)$.

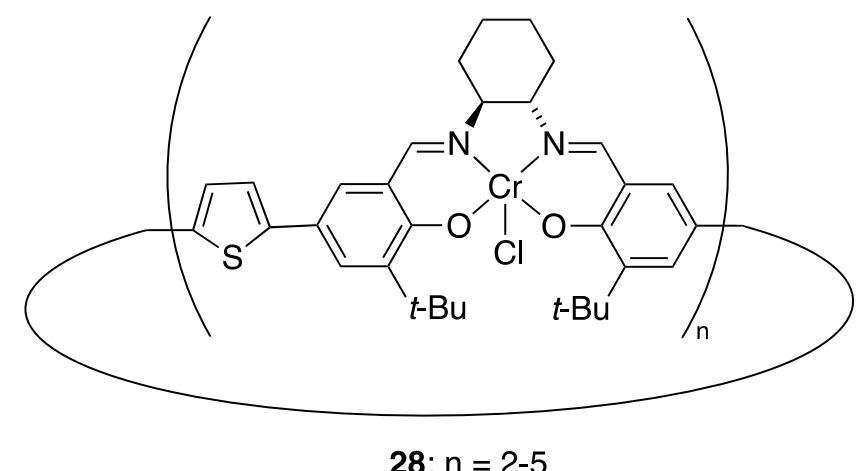

Figure 9. Macrocyclic calixsalen catalyst 28.

Inspired by Weck's [48] work on hydrolytic kinetic resolution (HKR) of terminal epoxides, Liu and coworkers [49] studied the catalytic properties of macrocyclic oligomer-supported $\mathrm{Cr}$-salen catalysts in the ARO of cyclohexene oxide with $\mathrm{TMSN}_{3}$. Catalysts with different linker lengths between the salen complex and the oligomers were synthesized and compared (catalysts 29-31, Figure 10). The catalysts were obtained as mixtures of different ring sizes (dimers to decamers) and used as such in the catalytic studies. Reactions carried out with $0.2 \mathrm{~mol} \%$ catalyst loading in diethyl ether gave significantly higher catalytic activity, enantioselectivity and reaction rates than for the monomeric catalyst $\mathbf{1}$ (Scheme 4 ) under the same conditions. The catalyst with the shortest linker (catalyst 29) showed the highest reaction rate (17 times higher initial TOF than monomeric catalyst 1 ) and the catalyst with the longest linker (catalyst 31) gave the highest enantioselectivity $(82 \% e e)$, demonstrating the importance of distance and relative orientation of the $\mathrm{Cr}$-salen complexes in multi-metallic catalysts. The catalysts could be recovered by precipitation with acetone and recycled up to five times with maintained reactivity and enantioselectivity.

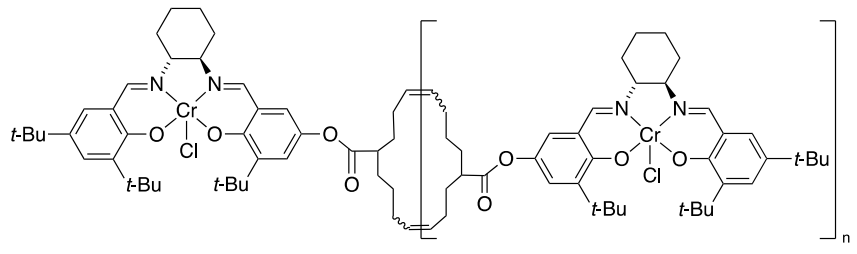

29: $n=1-9$

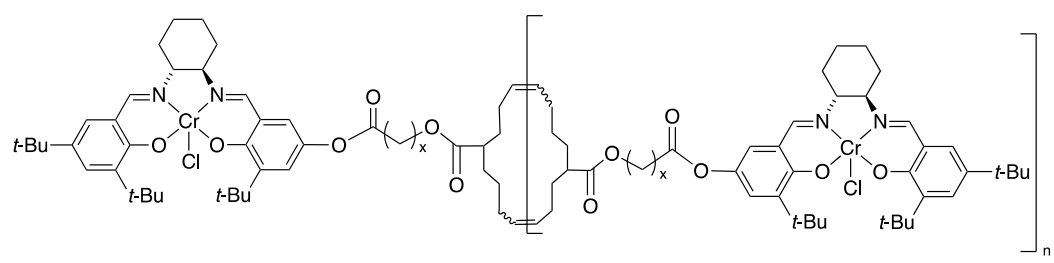

30: $x=3, n=1-8$
31: $x=7, n=1-7$

Figure 10. Macrocyclic oligomer-supported Cr-salen catalysts. 
In an effort to improve catalyst recycling, Song and coworkers [50] investigated the use of ionic liquids based on 1-butyl-3-methylimidazolium salts ([bmim][X], Figure 11) as reaction medium in the ARO of cyclic meso-epoxides with $\mathrm{TMSN}_{3}$ catalyzed by $\mathrm{Cr}$-salen complex $\mathbf{1}$ (Scheme 4). By performing the reactions in ionic liquids, the products could be extracted with hexane, while the catalyst remained in the ionic liquid phase. The recovered catalyst could be recycled several times without loss of reactivity or enantioselectivity. The study also found that the nature of the anionic counter ion has a large influence on the reaction, where reactions performed in $[\mathrm{bmim}]\left[\mathrm{PF}_{6}\right]$ gave high yields and enantioselectivies (Scheme 6), comparable to those obtained under homogeneous conditions [20], while reactions carried out in more hydrophilic ionic liquids like [bmim][OTf] hardly gave any product.

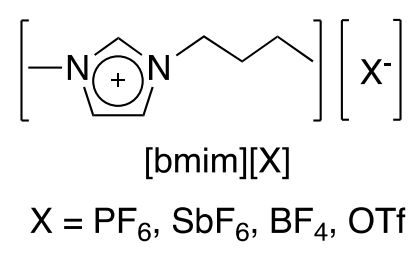

Figure 11. 1-Butyl-3-methylimidazolium salts.

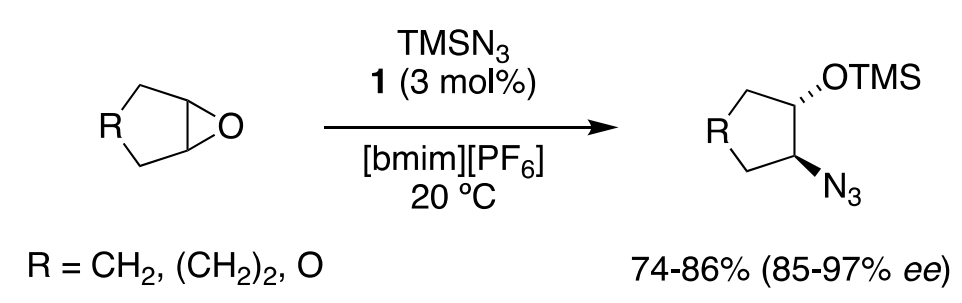

Scheme 6. The asymmetric azidolysis of cyclopentene oxide catalyzed by Cr-salen complex $\mathbf{1}$ (Scheme 4) in $[\mathrm{bmim}]\left[\mathrm{PF}_{6}\right]$.

Cui and coworkers [51] prepared chiral coordination cages with metal-salen linkers. The so formed supramolecular nanoreactors consisted of six dicarboxylate linkers based on metal-salen complexes (Mn(III)-, $\mathrm{Cr}(\mathrm{III})-$, and $\mathrm{Fe}(\mathrm{III})$ salen complexes) and four $\mathrm{Cp}_{3} \mathrm{Zr}_{3}$ cluster vertices, giving a tetrahedral cage with a hydrophobic cavity (Figure 12). Remarkably, the mixed-linker cage containing both Mn-salen and $\mathrm{Cr}$-salen linkers was able to catalyze the sequential asymmetric epoxidation/ring-opening of 2,2-dimethyl-2H-chromene, giving the product with high yield and enantioselectivity (Scheme 7). The cage catalyst also allowed for very low catalyst loadings and remained active at $0.005-0.01 \mathrm{~mol} \%$. The reactions were performed under homogeneous conditions, but the catalyst could be recovered by precipitation by the addition of diethyl ether and recycled up to five times with only a slight decrease of enantioselectivity. The same group also explored metal-organic frameworks (MOFs) containing metal-salen linkers prepared by post-synthetic exchange of ligands. The mixed $\mathrm{Cr}-\mathrm{Mn}$ MOFs catalyzed the sequential alkene epoxidation/ring-opening of 2,2-dimethyl-2H-chromene in good yield and enantioselectivity [52].

Yang and coworkers [53] used Cr-salen complex 1 (Scheme 4) to catalyze the ARO of meso-epoxides with $\mathrm{TMSN}_{3}$ as an example reaction to investigate the efficiency of their liquid-solid hybrid catalysts. The hybrid catalysts were designed to bridge homogeneous and heterogeneous catalysis and consisted of a catalyst-containing ionic liquid hosted in a porous solid outer crust (Figure 13). The ionic liquid phase consisted of $[\mathrm{bmim}]\left[\mathrm{PF}_{6}\right]$ mixed with $20 \%[\mathrm{bmim}]\left[\mathrm{BF}_{4}\right]$ and a small amount of water, and the silica porous crust was prepared from tetramethoxysilane (TMOS). The catalytic particles were successfully packed into fixed-bed reactors for continuous flow reactions. During the reaction, the reactants could pass through the porous crust to the liquid pool where the "homogeneous" reaction occurred. When used in a continuous flow system with $n$-octane as the mobile phase, the hybrid catalyst demonstrated high catalytic efficiency for the ARO of cyclopentene oxide, cyclohexene oxide and cis-2,3-epoxybutane. The products were obtained with excellent yields (>99\%) and up to $93 \%$ ee 
(for the product from cyclopentene oxide). The reactivity and enantioselectivity could be maintained over $600 \mathrm{~h}$.

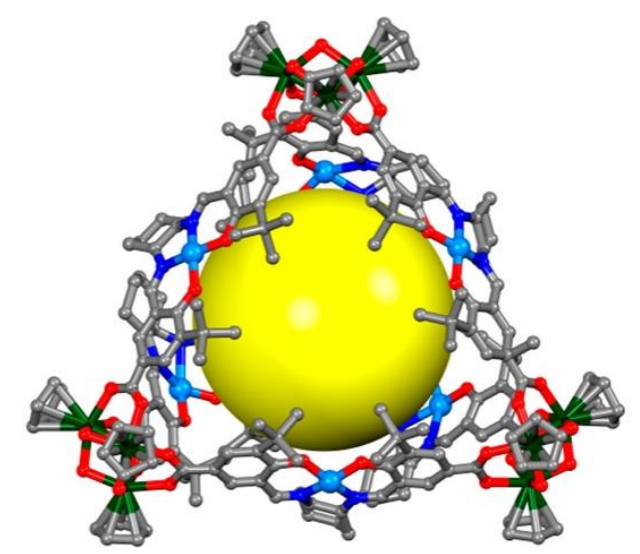

Figure 12. Single crystal X-ray structure of Mn-salen linked coordination cage. The yellow sphere highlights the hydrophobic cavity. Reprinted with permission from Reference [51]. Copyright 2018 American Chemical Society.

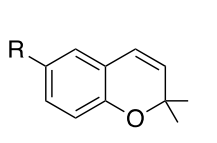

$\mathrm{R}=\mathrm{H}, \mathrm{Me}, \mathrm{NO}_{2}$

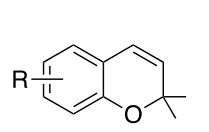

$\mathrm{R}=\mathrm{H}, \mathrm{MeO}, \mathrm{NO}_{2}$
1) 2-(tert-butylsulfonyl)iodosylbenzene cat. $(0.2 \mathrm{~mol} \%), \mathrm{CH}_{2} \mathrm{Cl}_{2}, 0^{\circ} \mathrm{C}, 8 \mathrm{~h}$ 2) $\mathrm{TMSN}_{3}(1.1 \mathrm{eq}), 0^{\circ} \mathrm{C}, 16 \mathrm{~h}$

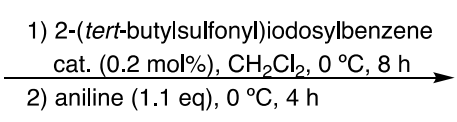<smiles>[R]O[C@H]1[C@H](N)c2cc([R])ccc2OC1(C)C</smiles>

$60-82 \%(91-99.9 \%$ ee $)$

Scheme 7. The sequential asymmetric epoxidation/ring-opening of 2,2-dimethyl-2H-chromenes catalyzed by the mixed Mn-Cr coordination cage shown in Figure 12.

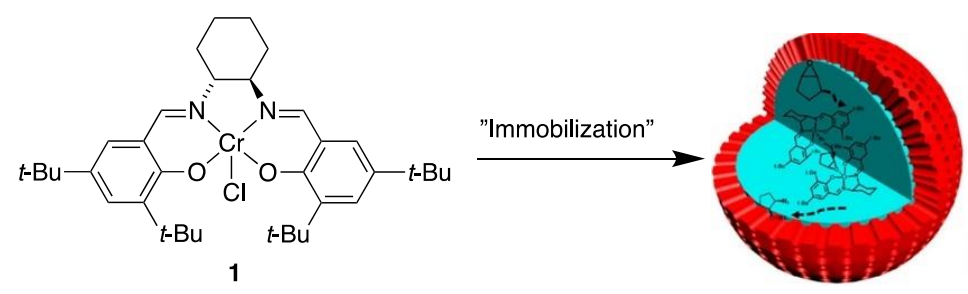

Figure 13. A liquid-solid hybrid catalyst. Adapted with permission from Reference [53]. Copyright 2019 American Chemical Society.

\subsection{With Anilines, Amines, and Carbamates}

An alternative, seemingly more straightforward method for obtaining enantiopure vicinal amino alcohols than first forming the azido alcohol and then reducing it, is to use amines as nucleophiles in the ARO of meso-epoxides. However, this approach suffers from an inherent compatibility problem, as the Lewis acidic metal-salen catalyst can be deactivated by complexation with the Lewis basic amine. Nevertheless, there are several examples of successful strategies for overcoming these issues and achieving highly active and enantioselective catalysis of the aminolysis of meso-epoxides.

Bartoli and Melchiorre [54] employed Cr-salen complex 1 (Scheme 4) in the ARO of meso-stilbene oxide with anilines as nucleophiles. Reactions carried out in dichloromethane with $10 \mathrm{~mol} \%$ catalyst loading afforded the corresponding amino alcohols with high yields, complete diastereocontrol (only 
the syn-isomer was observed) and high enantioselectivity (Scheme 8). The use of a catalytic amount of $\mathrm{Et}_{3} \mathrm{~N}$ as an additive was found to give a significantly enhancement of the enantioselectivity (from $76 \%$ to $90 \%$ ee for reaction with aniline) but caused a moderate loss of reactivity.

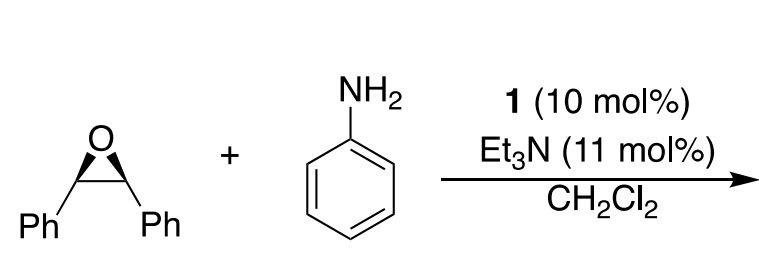<smiles>OC(c1ccccc1)[C@H](Nc1ccccc1)c1ccccc1</smiles>

$98 \%(90 \%$ ee $)$

Scheme 8. The asymmetric aminolysis of meso-stilbene catalyzed by Cr-salen complex 1 (Scheme 4).

Kureshy and coworkers $[55,56]$ investigated several different approaches to achieve the enantioselective synthesis of vicinal amino alcohols by the ARO of meso-epoxides. One such approach is the use of in situ generated monomeric and polymeric Ti(IV)salen complexes from ligands 32 and 33 (Scheme 9) [55]. In order to achieve an efficient asymmetric catalysis, high catalyst loading $(20 \mathrm{~mol} \%)$ and the addition of additives was required. For the ARO of meso-stilbene oxide with aniline, the best results were obtained with the enantiopure imine additive A (Scheme 9). Under optimized conditions, the ring-opened product was obtained in excellent yield and enantioselectivity ( $99 \%$ yield and $>99 \%$ ee). Noticeably, the use of the opposite enantiomer of the additive resulted in significantly lower enantioselectivity $(75 \% \mathrm{ee})$. The authors suggested that this could indicate synergistic effects between the catalyst and the additive but did not investigate it further. For the ARO of cyclic epoxides, triphenylphosphine was found to be the best additive, although yields and ee values were significantly lower than those found for meso-stilbene oxide. The monomeric and polymeric catalyst gave comparable yields and enantioselectivities, but the polymeric catalyst generated from ligand 33 showed higher reactivity. In addition, the polymeric catalyst could be precipitated out from the reaction mixture by the addition of $n$-hexane and recycled several times without loss of enantioselectivity.
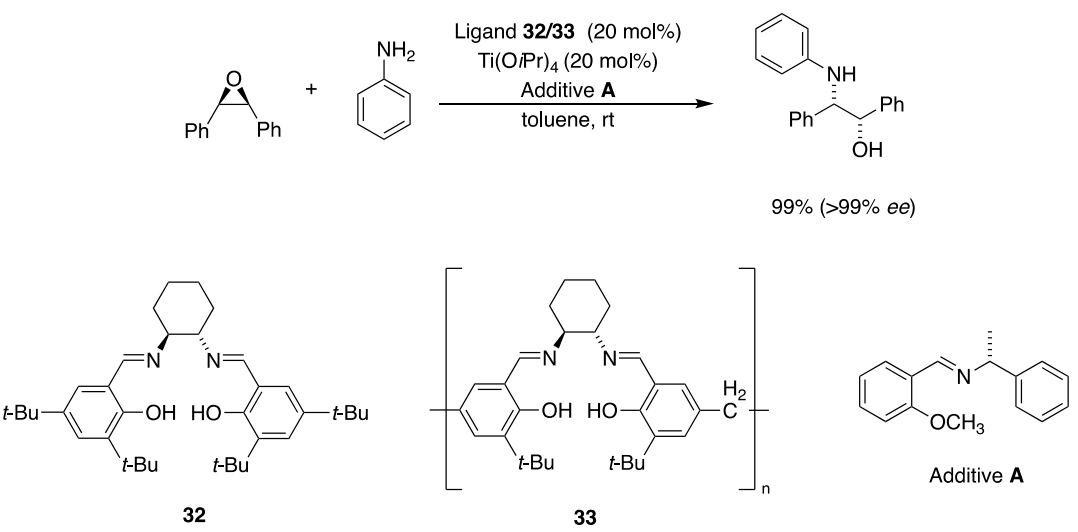

Scheme 9. The asymmetric aminolysis of meso-stilbene oxide catalyzed by in situ formed Ti-salen complexes using ligands 32 and 33.

The same group also synthesized and evaluated a series of enantiopure macrocyclic Cr-salen complexes with different counter ions [56]. After the screening of different reaction conditions, optimal results were obtained for reactions performed in $\mathrm{CH}_{2} \mathrm{Cl}_{2} / \mathrm{MeOH}(9: 1 v / v)$ with $0.5 \mathrm{~mol} \%$ catalyst loading using catalyst 34 (Figure 14). These conditions were used for the ARO of a limited number of cyclic and acyclic meso-epoxides with aniline, giving the products in excellent yields (98-99\%) and high enantioselectivities (up to $91 \% \mathrm{ee}$ ). The catalyst could be recycled up to four times without loss of reactivity or enantioselectivity. 


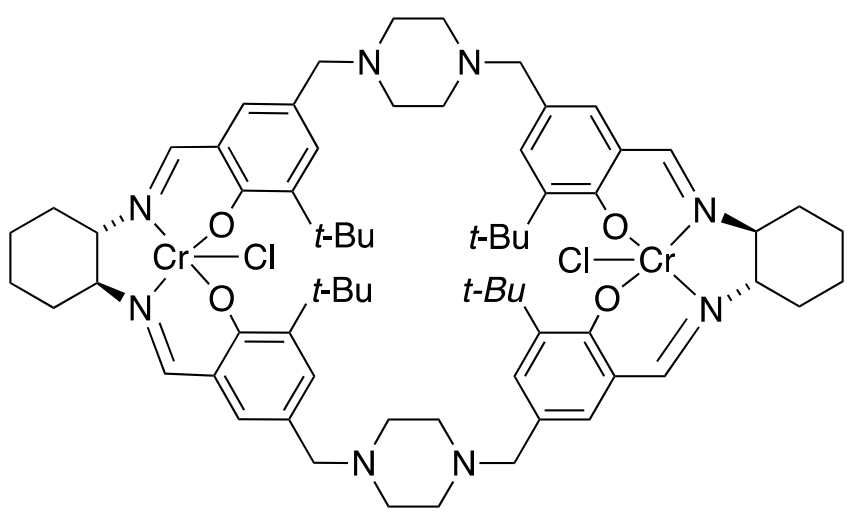

34

Figure 14. Macrocyclic Cr-salen complex 34.

Jacobsen and coworkers $[57,58]$ used cyclic Co-salen catalyst 35 as a mixture of oligomers in the ARO of cyclic meso-epoxides with phenyl carbamate, resulting in the asymmetric synthesis of $N$-protected trans-1,2-amino alcohols in high yields and with excellent enantioselectivities (Scheme 10). For six-membered ring epoxides, the addition of the nucleophile was followed by intramolecular cyclization, resulting in trans-4,5-disubstituted oxazolidinone products. The ARO of five-membered ring epoxides also proceeded smoothly, although the products did not undergo cyclization, most likely due to the unfavorable strain in trans-fused 5-5 ring systems. The products could be deprotected by hydrolysis under basic conditions, and the method could be scaled-up to prepare multigram quantities of the products (Scheme 11).

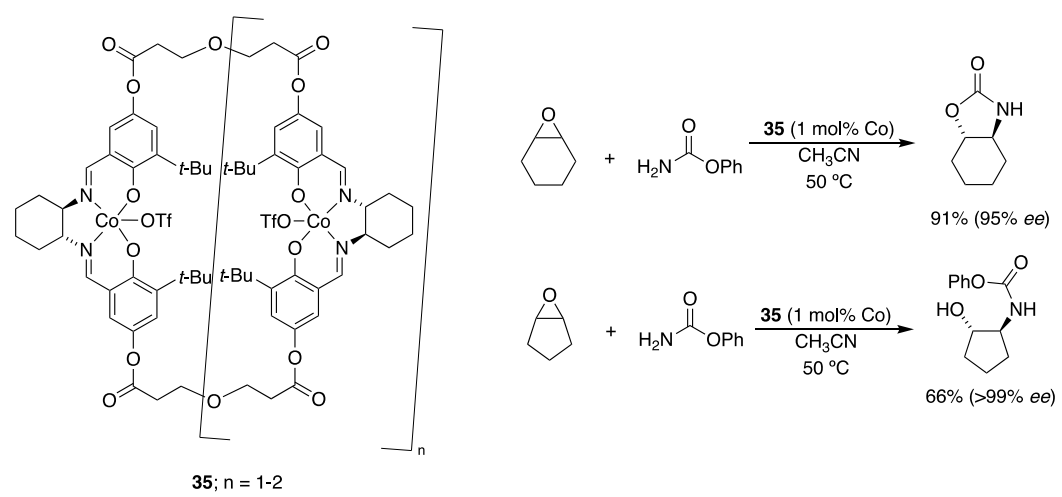

Scheme 10. The asymmetric carbamolysis of meso-epoxides catalyzed by cyclic oligomeric Co-salen complex 35.

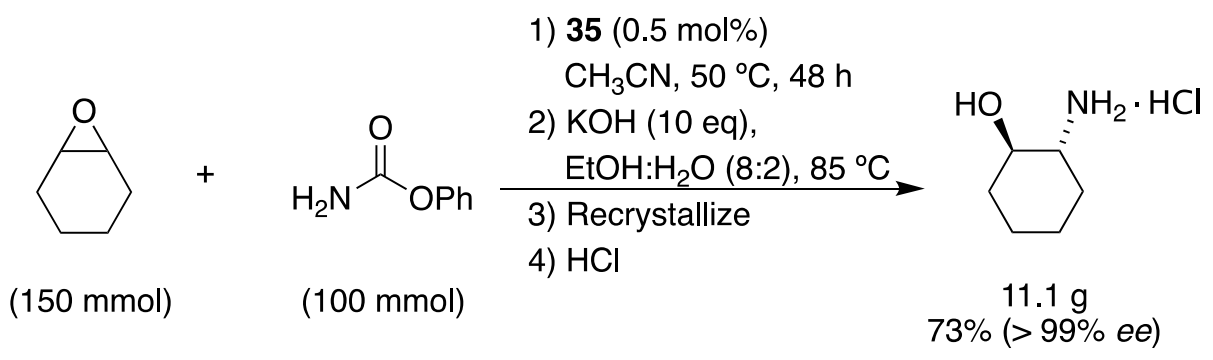

Scheme 11. A multigram synthesis of trans-2-aminocyclohexanol hydrochloride using oligomeric catalyst 35 (Scheme 10).

Peddinti and coworkers [59] used Co(III)salen complexes to catalyze the ARO of cyclohexene oxide with secondary aliphatic amines, enabling the highly enantioselective synthesis of biologically 
important molecules such as vesamicol. Screening of reaction conditions revealed that the solvent had a large impact on the reaction rate and the enantioselectivity. The counter ion was also shown to affect both the catalytic activity of the catalyst and the enantioselectivity of the reaction. For the ARO of cyclohexene oxide with 4-arylpiperidines, the reaction performed with catalyst 36 in tert-butyl methyl ether (TBME) gave products with good yields and good to excellent enantioselectivities (Scheme 12).
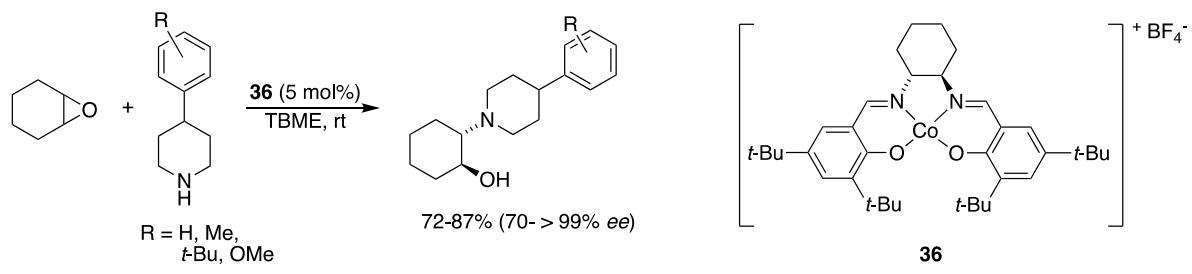

Scheme 12. The asymmetric aminolysis of cyclohexene oxide catalyzed by Co-salen complex 36 .

In the area of heterogeneous catalysis, Islam and coworkers developed catalysts based on a series of metal-salen complexes supported on functionalized mesoporous silica materials. A chiral Fe(III)salen complex was covalently immobilized on mesoporous silica SBA-15 through an aminopropyl linker (Figure 15) [60]. The so-formed heterogeneous catalyst 37 was used in the ARO of cyclohexene oxide with different anilines, giving the products in high yields (85-96\%) and with excellent enantioselectivities (96-99\% ee). Notably, the reactions could be performed under solvent-free conditions and reached complete conversions in just $2-3 \mathrm{~h}$ at room temperature. The catalyst could be recycled up to five times with maintained reactivity and enantioselectivity.

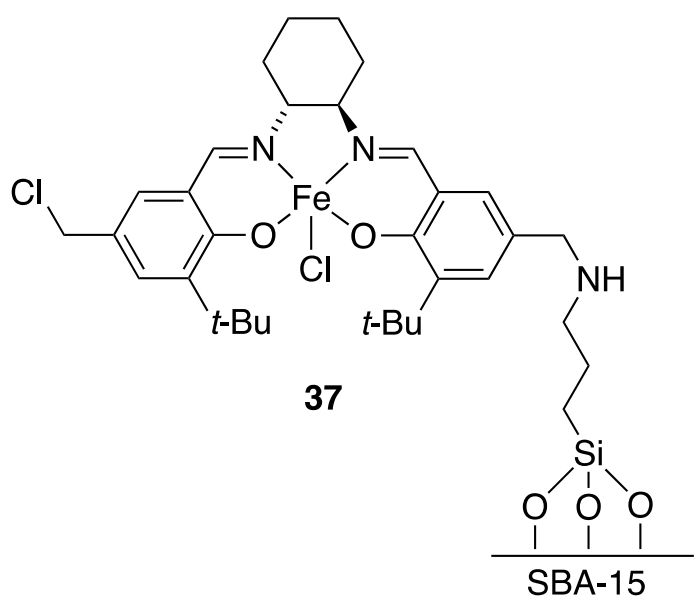

Figure 15. A Fe-salen complex immobilized on mesoporous silica SBA-15.

Islam and coworkers [61] also immobilized Co-salen complexes on mesoporous silica. The Co-salen complexes were grafted on the material through non-covalent interactions between quaternary amine groups on the salen units and carboxylate units on the functionalized silica (catalyst 38, Figure 16). The material showed excellent catalytic activities for the ARO of cyclohexene oxide with a number of aromatic and cyclic amines under solvent-free conditions, with short reaction times (1-2.5 h), high yields and high enantioselectivities (87-97\% yield and 77-99\% ee). After the reaction, the catalyst could be recovered by precipitation and centrifugation and then recycled without loss of reactivity or enantioselectivity and with no detectable metal leaching. 


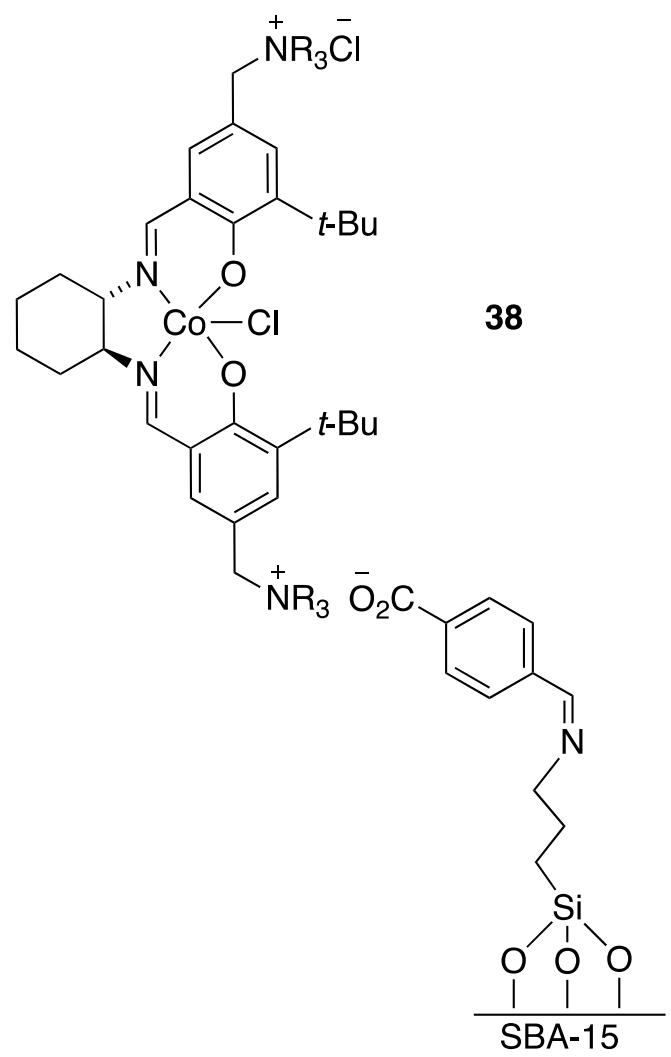

Figure 16. A Co-salen complex immobilized on mesoporous silica SBA-15.

Tu and coworkers [62] developed two-dimensional self-supported (i.e., immobilized without the use of an external support) chiral catalysts based on titanium. The catalytic systems consisted of coordination assemblies consisting of heteroditopic ligands containing enantiopure 1,1'-bi-2-naphthol (BINOL) and salen derivatives, where the oxygen bridge in dimeric Ti(IV)salen complexes was used as a crosslinker (Figure 17). Due to the fact of their insolubility in most organic solvents and water, the assemblies were used as heterogeneous catalysts in the ARO of cyclic and acyclic meso-epoxides with benzylic and aliphatic amines as nucleophiles. The reactions were performed in toluene and the products were obtained in high yields and enantioselectivities (83-99\% yield and $83-98 \%$ ee). Furthermore, saturated analogues of the metal-salen complexes were also investigated, and the salan-based catalyst 39 was successfully used for the one-pot sequential asymmetric epoxidation/ring-opening of 2,2-dimethyl-4,7-dihydro-1,3-dioxepine, giving the product with high yield and enantioselectivity (Scheme 13). The self-supported catalysts demonstrated very high stability and could be reused up to 20 times without significant loss in yield or enantioselectivity.

Cui and coworkers [63] developed heterogeneous catalysts based on MOFs and coordination cages with metal-salen linkers. The $\mathrm{Cr} / \mathrm{VO}$-salen mixed MOF catalysts were fabricated via solvent-assisted linker exchange and used in the ARO of meso-stilbene oxide with aniline as nucleophile. The reaction performed in $\mathrm{CH}_{2} \mathrm{Cl}_{2}$ with $5 \mathrm{~mol} \%$ catalyst loading gave the product in $87 \%$ yield and $76 \%$ ee. The same group also designed coordination cages with mixed $\mathrm{Mn}$-salen and $\mathrm{Cr}$-salen linkers which were able to catalyze the sequential asymmetric epoxidation/ring-opening of 2,2-dimethyl-2H-chromene with anilines, affording the products in good yield (60-82\%) and with excellent enantioselectivity (up to $99.9 \%$ ee) (Scheme 7) [51]. 
(a)

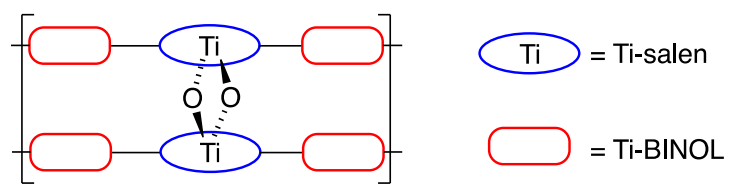

(b)

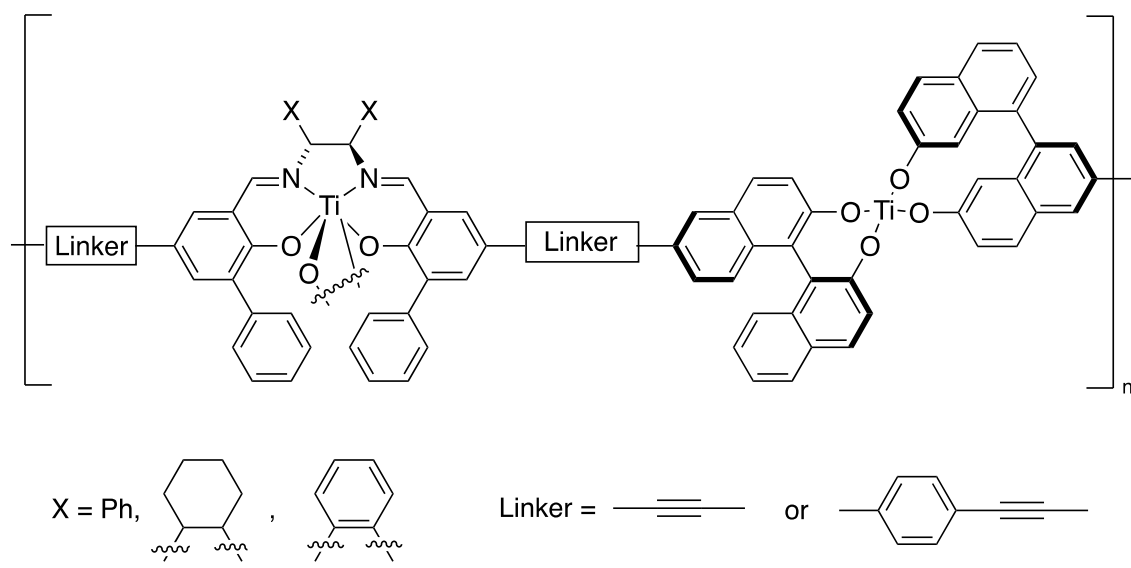

Figure 17. (a) A schematic representation of 2D titanium metal-organic coordination assemblies. (b) A self-supported chiral Ti-salen catalyst with different linkers and diamine substituents.
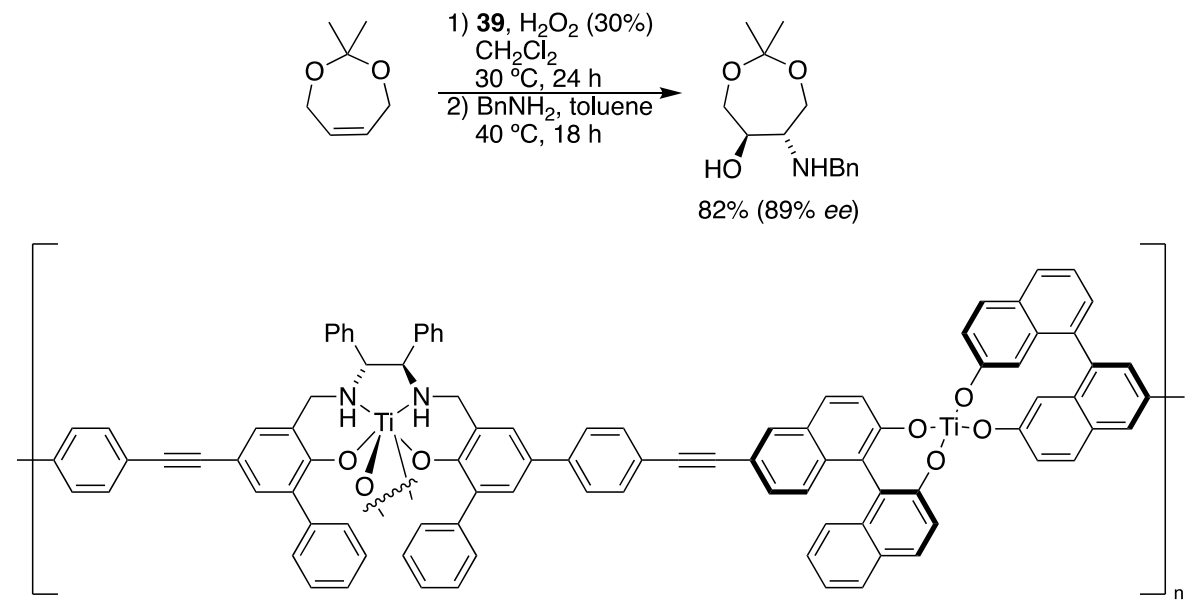

39

Scheme 13. The sequential asymmetric epoxidation/ring-opening of 2,2-dimethyl-4,7-dihydro-1,3dioxepine catalyzed by self-supported Ti-salan catalyst 39 .

\subsection{With Oxygen-Containing Nucleophiles}

The hydrolytic asymmetric ring opening of cyclic meso-epoxides provides a pathway towards enantioenriched vicinal trans-diols and is a valuable complement to other methods such as the catalytic asymmetric dihydroxylation of cyclic alkenes which selectively produce cis-diols [64]. Several of the catalysts that have proven effective for the HKR of terminal epoxides have also been investigated in the ARO of meso-epoxides [65-68]. While many of the HKR catalysts failed to exhibit high reactivity and enantioselectivity in the ARO of meso-epoxides, there are a few examples where the use of Co(III)salen complexes afforded enantioenriched vicinal trans-diols in high yields and enantioselectivities.

In 2001, Jacobsen and coworkers [69] reported a mixture of macrocyclic oligosalen complexes (catalyst 40, Figure 18) as a catalyst for the HKR of terminal epoxides. This catalyst was also proven to 
be effective in the hydrolytic ARO of cyclohexene oxide. Using this catalyst, trans-1,2-cyclohexane diol was synthesized with $98 \%$ yield and $94 \%$ ee (Scheme 14).
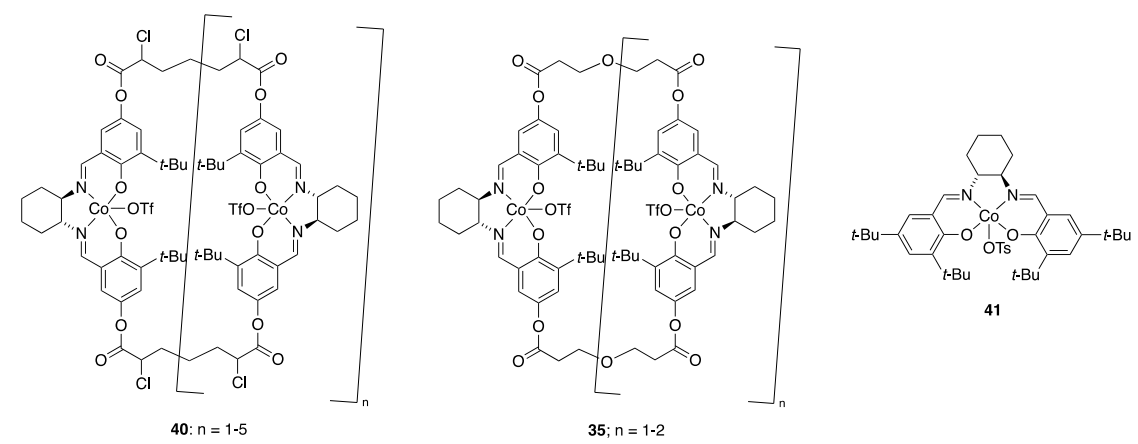

Figure 18. Macrocyclic oligosalen mixtures 40 and 35 and monomeric analogue 41.

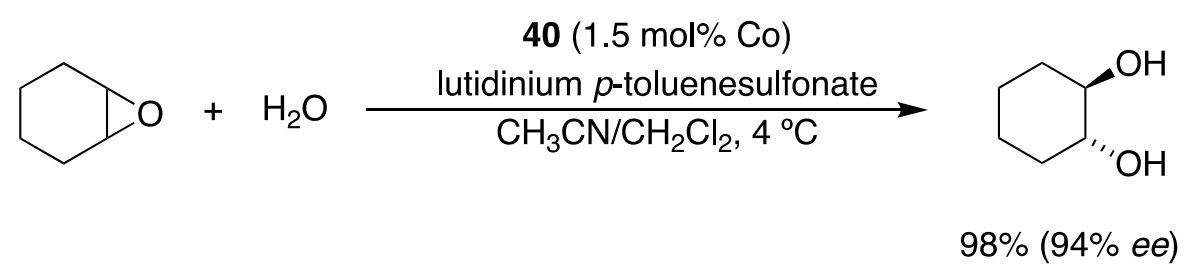

Scheme 14. The asymmetric hydrolysis of cyclohexene oxide catalyzed by cyclic oligomeric Co-salen complex 40 (Figure 18).

Kinetic studies demonstrated that oligosalen catalyst 40 was much more reactive and enantioselective in the hydrolysis of cyclohexene oxide than monomeric analogue 41 (Figure 18). Despite its excellent reactivity, the application of catalyst $\mathbf{4 0}$ was limited by its low solubility in the reaction mixture. In addition, reproducibility problems were observed when applying $\mathbf{4 0}$ from different batches in the same reaction [69]. To resolve these issues, the same group reported a new generation of oligomeric salen complexes (catalyst 35 in Figure 18) which could be prepared on a multigram scale with $60-66 \%$ overall yield [58]. The mixture was found to consist mainly of dimer $(n=1)$, with a small amount of trimer $(n=2)$. The catalyst showed improved reactivity and enantioselectivity compared to monosalen complex $\mathbf{4 1}$ and oligomeric mixture $\mathbf{4 0}$ in the hydrolysis of meso-epoxides. The catalyst tolerated a broad scope of substrates and gave the ring-opened products in high yields and enantioselectivities for five- and six-membered cyclic meso-epoxides (Scheme 15). The enhanced reactivity of these oligomeric salen complexes compared to monosalen analogues was attributed to the cooperative intramolecular interactions between two salen moieties, which was further supported by the observed first-order kinetic dependence on catalyst concentration.

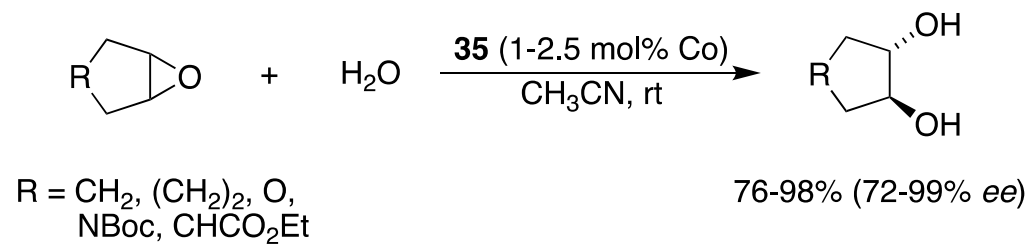

Scheme 15. The asymmetric hydrolysis of cyclic meso-epoxides catalyzed by cyclic oligomeric Co-salen complex 35 (Figure 18).

The cooperative mechanism was also exploited by Liu and coworkers in the design of bimetallic catalyst 42 (Figure 19) [70]. Complex 42 is capable of self-assembling into a dimer by aromatic donor-acceptor interactions between naphthalenediimide and pyrene. The catalyst displayed excellent reactivities in HKR reactions of several terminal epoxides as well as in the hydrolytic ARO of 
cyclohexene oxide, the latter detailed in Scheme 16. In addition, it was found that performing the reactions with a 1:1 mixture of analogues 43 and 44 (Figure 19) gave similar or better results than complex 42. Using only one of the analogues (either 43 or 44) required significantly longer reaction times to reach full conversions. This further supported that the aromatic donor-acceptor interactions played an important role for the reactivities of these catalysts.
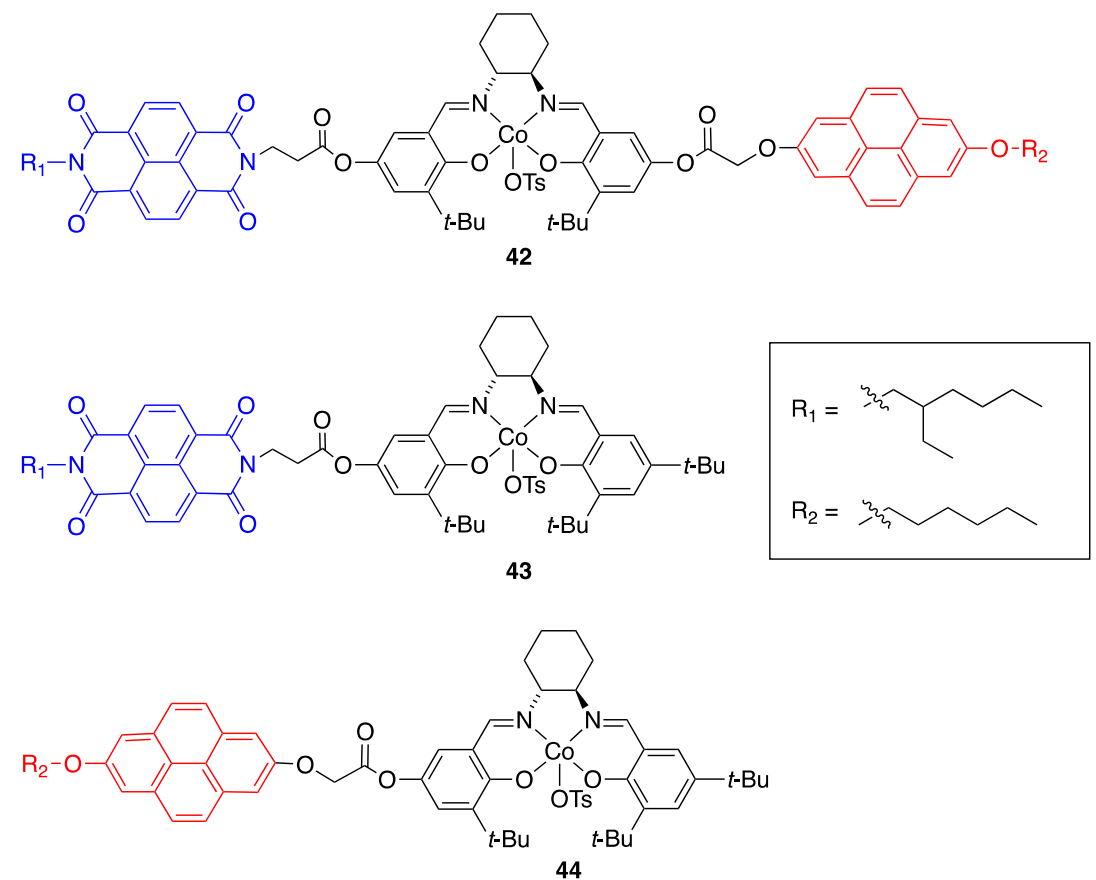

Figure 19. Self-assembling cooperative Co-salen catalysts $42-44$ (blue $=$ aromatic acceptor, red $=$ aromatic donor).

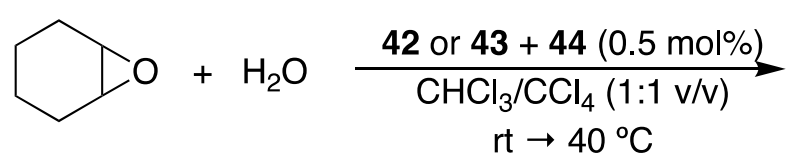<smiles>OC1CCCCC1O</smiles>

42: $89 \%(85 \%$ ee $)$

$43+44: 88 \%(84 \%$ ee $)$

Scheme 16. The asymmetric hydrolysis of cyclohexene oxide catalyzed by cooperative Co-salen complexes $\mathbf{4 2}$ or $\mathbf{4 3}+\mathbf{4 4}$ (Figure 19).

The ARO of meso-epoxides with carboxylic acids is of interest owing to its possibility to access monoesters of vicinal diols in high yield and with high enantio- and diastereoselectivity, something which is difficult to achieve with other synthetic methods. Jacobsen's group investigated this type of reaction with various monosalen catalysts, finding that a $\mathrm{Co}(\mathrm{III})$ salen complex, generated in situ by stirring $\mathrm{Co}$ (II)salen complex 45 (Figure 20) in carboxylic acid in air, was the most effective in catalyzing the reaction [71]. With the addition of diisopropylethylamine, complex 45 catalyzed the reactions between meso-epoxides and benzoic acid, affording the corresponding mono-benzoate esters in good to excellent yields and ee values (Scheme 17). The substrate scope included cyclic and acyclic meso-epoxides with aliphatic and aromatic substituents. The use of one equivalent of weakly coordinating base was necessary to achieve a high rate, enantioselectivity and yield. The authors hypothesized that the beneficial effects might stem from the fact that amines significantly increase the solubility of benzoic acid in TBME. The crystallinity of the mono-benzoate esters also meant that for reactions with moderate ee values, the enantiomeric excess could easily be further improved by recrystallization. This methodology was successfully applied to the ARO of meso-epoxides with 
benzoic acid and benzoic acid derivatives as nucleophiles. Using other acids, such as acetic acid or pivalic acid, resulted in lower enantioselectivity and reactivity and was not pursued further.

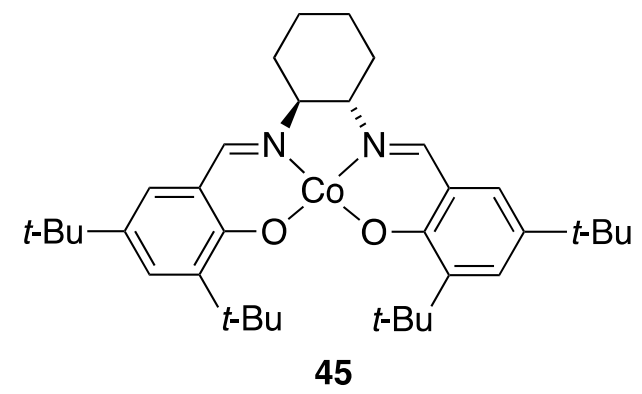

Figure 20. Co(II)salen complex 45.

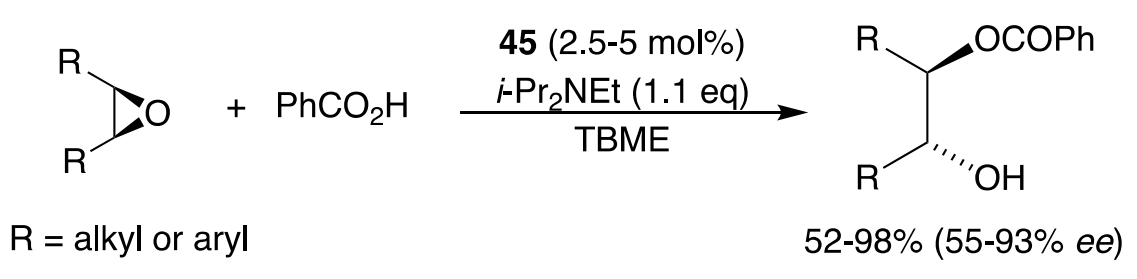

Scheme 17. The asymmetric ring-opening of meso-epoxides with benzoic acid catalyzed by Co-salen complex 45 (Figure 20) to generate monoesters.

\subsection{With Halogens}

Metal-salen complexes have seen limited use as catalysts for the ARO of meso-epoxides with halogen nucleophiles. The few examples that can be found in literature are focused on fluorinations, most likely owing to the growing interest in and importance of fluorine substituted organic compounds [72-74].

Haufe and coworkers published a number of studies using Cr-salen complex 46 and different fluoride sources to achieve the enantioselective fluorination of epoxides. The reaction of cyclohexene oxide with $\mathrm{KHF}_{2} / 18$-crown-6 gave $(R, R)$-2-fluorocyclohexanol in $64 \%$ yield and $55 \%$ ee when stoichiometric amounts of catalyst $\mathbf{4 6}$ was used. Attempts to lower the catalyst loading resulted in a drastic drop in enantioselectivity. The reaction also produced some amount of the corresponding chlorohydrin [75]. Changing the fluoride source to silver fluoride led to improved yields and enantioselectivities, and complete suppression of the formation of the chlorinated by-product, although a high catalyst loading of 50-100 mol\% was still required (Scheme 18) [76]. Attempts to lower the necessary quantity of catalyst by using different fluoride sources and reaction conditions proved unsuccessful [77].
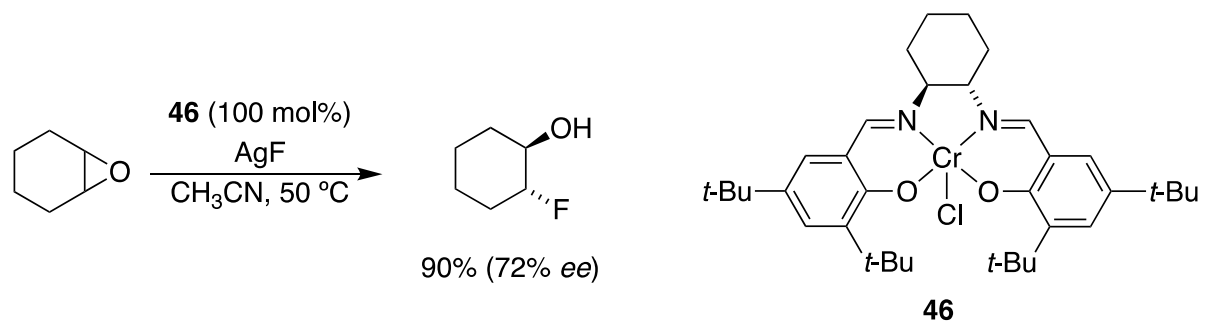

Scheme 18. The asymmetric ring-opening of cyclohexene oxide with AgF catalyzed by $\mathrm{Cr}-\mathrm{salen}$ complex 46.

Doyle and Kalow reported the enantioselective ring-opening of cyclic meso-epoxides with fluoride catalyzed by $\mathrm{Co}(\mathrm{II})$ salen complex 47 and an enantiopure amine cocatalyst. The protocol included in 
situ generation of HF from benzoyl fluoride and 1,1,1,3,3,3-hexafluoro-2-propanol (HFIP). For the ARO of variety cyclic meso-epoxides, the use of (-)-tetramisole as the chiral base gave good yields and high enantioselectivities of the resulting fluorohydrins (Scheme 19) [78].

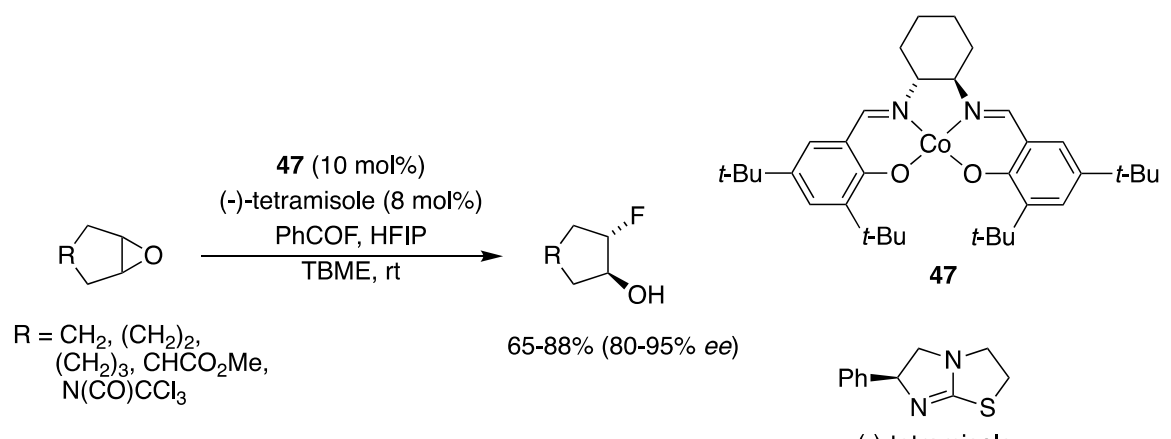

(-)-tetramisole

Scheme 19. The asymmetric ring-opening of meso-epoxides with HF catalyzed by Co(II)salen complex 47.

Extensive mechanistic studies of the above reaction resulted in some unexpected and seemingly incompatible data. Kinetic studies demonstrated an apparent first-order dependence on the catalyst while substituent and nonlinear effects supported a bimetallic rate-determining step. Based on this, the authors proposed that the active fluorinating agent was a cobalt-bifluoride complex which formed a fluorine-bridged dimer as a resting state. The amine cocatalyst was proposed to facilitate the dissociation of the dimer (Scheme 20). Further support for the proposed bimetallic mechanism was obtained by the use of the covalently linked Co(II)salen dimer 48 (Figure 21), which resulted in increased reaction rates, extended substrate scope and high enantioselectivity [79].

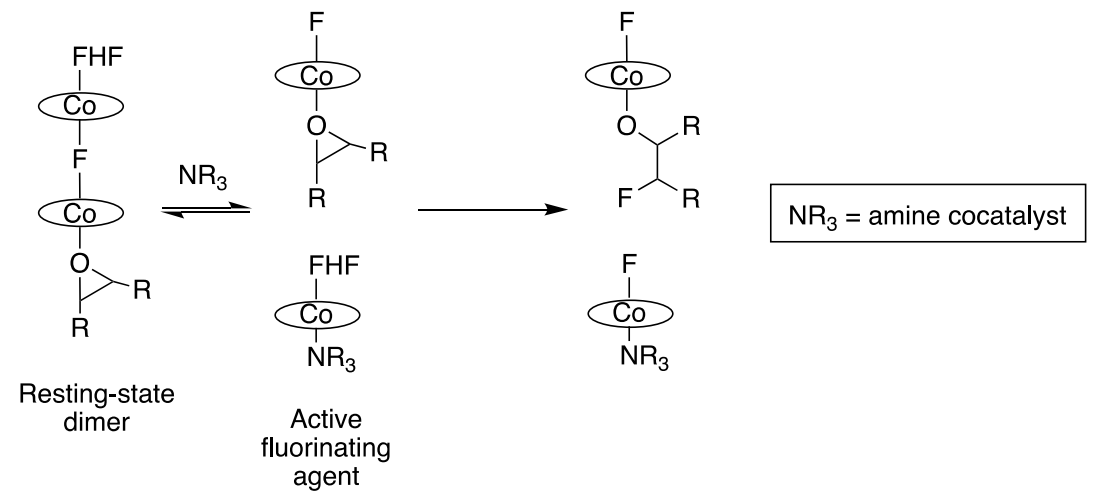

Scheme 20. A part of the proposed mechanism of the asymmetric fluorination of meso-epoxides catalyzed by Co-salen 47 (Scheme 19), proposed by Doyle and Kalow [79], showing the dimeric resting-state and its dissociated form.

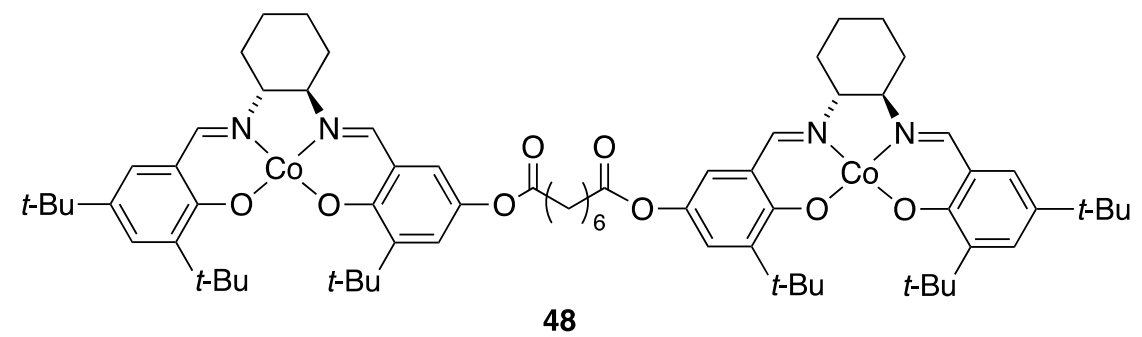

Figure 21. Dimeric Co(II)salen catalyst 48. 


\subsection{With Thiols and Selenols}

Jacobsen and coworkers applied Cr-salen catalyst 46 (Scheme 18) in the ARO of cyclohexene oxide with benzyl mercaptan as the nucleophile (Scheme 21) [80]. The product was obtained in good yield but with only moderate enantioselectivity. The enantioselectivity could be improved by instead employing 1,4-benzenedimethanethiol as the nucleophile, which resulted in a diastereomeric mixture of $C_{2}$-symmetric and meso ring-opened products (Scheme 22). The desired $C_{2}$-symmetric product could be isolated with high enantioselectivity and transformed into the free thiol by a dissolving metal reduction in a subsequent step.

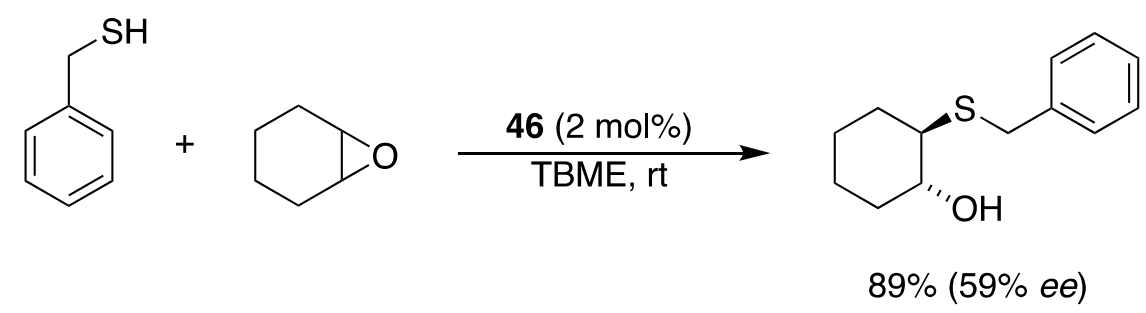

Scheme 21. The asymmetric ring-opening of cyclohexene oxide with benzyl mercaptan catalyzed by Cr-salen complex 46 (Scheme 18).
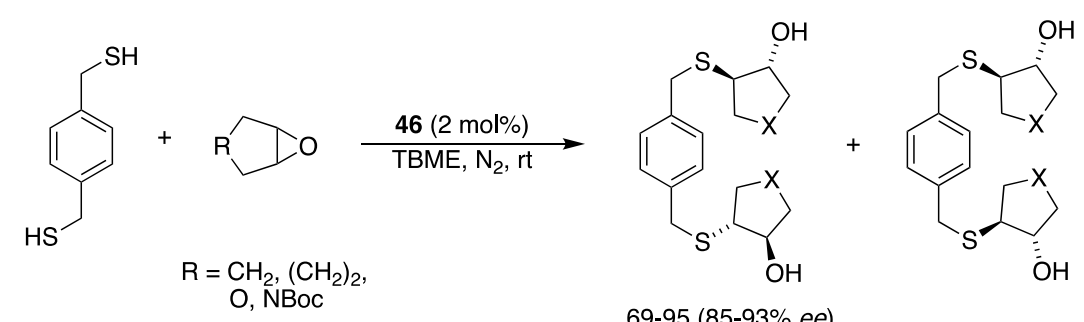

Scheme 22. The asymmetric ring-opening of meso-epoxides with 1,4-benzenedimethanethiol catalyzed by $\mathrm{Cr}$-salen complex 46 (Scheme 18).

Many of the reported methods for the ARO of meso-epoxides with thiols and selenols employ Ti-salen catalysts. One such method was published by Hou and coworker, who used a chiral Ti(IV)salen complex, formed in situ from $\mathrm{Ti}(\mathrm{OiPr})_{4}$ and salen ligand 32 (Scheme 9). This catalyst afforded vicinal hydroxy sulfides in good yields and moderate enantioselectivities (Scheme 23) [81]. The choice of sulphur nucleophile could also be extended to dithiophosphorus acids, which was explored by Zhou and Tang $[82,83]$. Their protocol gave the ring-opened product in high yield and good enantioselectivity. Subsequent reduction afforded the corresponding vicinal hydroxy sulfide (Scheme 24).

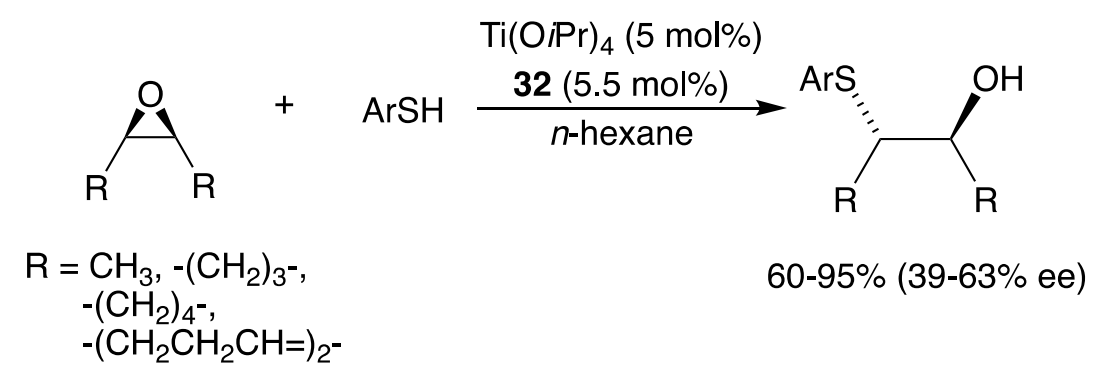

Scheme 23. The asymmetric ring-opening of meso-epoxides with thiols catalyzed by a Ti-salen complex generated in situ from $\mathrm{Ti}(\mathrm{O} i \mathrm{Pr})_{4}$ and salen ligand 32 (Scheme 9). 


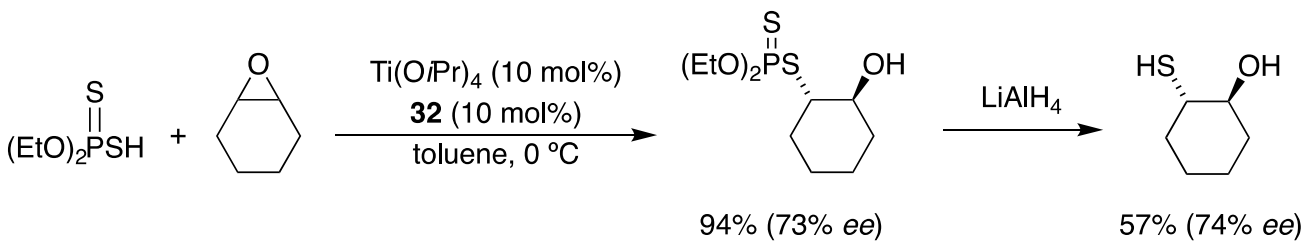

Scheme 24. The asymmetric ring-opening of cyclohexene oxide with dithiophosphorous acid catalyzed by a Ti-salen complex generated in situ from $\mathrm{Ti}(\mathrm{OiPr})_{4}$ and salen ligand 32 (Scheme 9).

For the enantioselective addition of aryl selenols to meso-epoxides, Zhu and coworkers reported the use of heterobimetallic titanium-gallium-salen complex 49 (Scheme 25) [84]. The "open" complexes were prepared by first incorporating gallium $\left(\mathrm{GaMe}_{3}\right)$ and then adding $\mathrm{Ti}(\mathrm{OiPr})_{4}$. Catalyst 49 was used in the ARO of a number of cyclic and acyclic meso-epoxides with aryl selenols as nucleophiles, affording the products in high yields and up to $97 \%$ ee. The authors proposed a strong synergistic effect between the two Lewis acids, with the hard Lewis acid titanium activating the epoxide and the softer gallium coordinating the arylselenol and directing the nucleophilic attack. The same method could also be extended to the ARO of meso-epoxides with thiols, resulting in high yields and moderate to high enantioselectivities (up to $92 \%$ ee) [85,86].
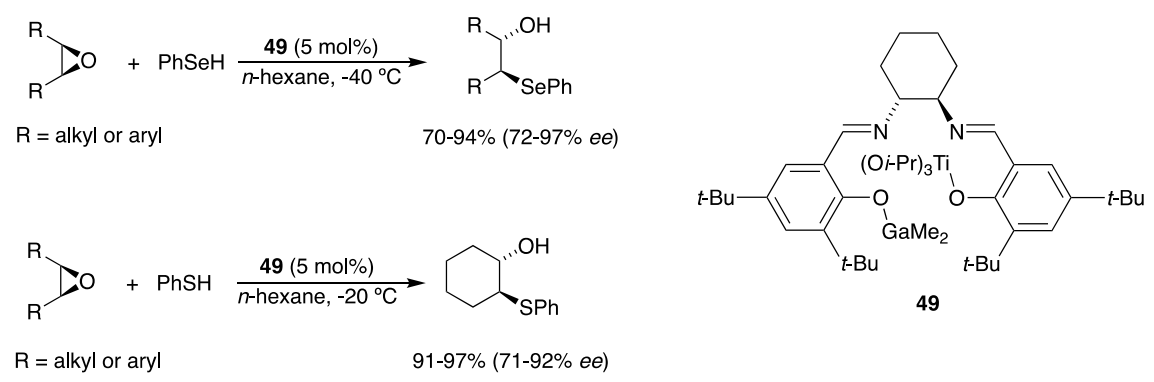

Scheme 25. The asymmetric ring-opening of meso-epoxide with aryl selenols and thiols catalyzed by Ti-Ga-salen complex 49.

Another protocol for the asymmetric synthesis of vicinal hydroxy selenides was reported by Tiecco and Marini, who used Cr-salen complexes to catalyze the ARO of meso-epoxides with (phenylseleno)silanes as nucleophiles [87]. Complexes with different counter ions, reactions in different solvents and with different additives were evaluated. The best results were obtained with $5 \mathrm{~mol} \%$ of complex $\mathbf{5 0}$ with $\mathrm{BF}_{4}{ }^{-}$as counterion in TBME in the presence of one equivalent of $N, N, N^{\prime}, N^{\prime}$-tetramethylethylenediamine (TMEDA) at $-10^{\circ} \mathrm{C}$, which gave the products in good to excellent yields and enantioselectivities (Scheme 26). The method gave better results for meso-stilbene oxide and its derivatives than for cyclic meso-epoxides.

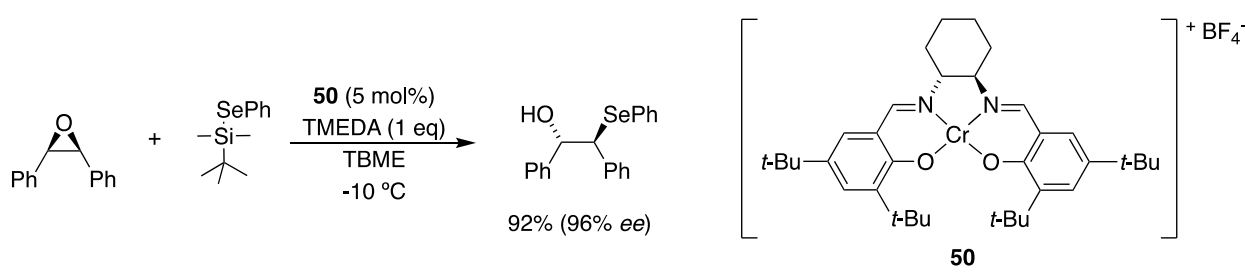

Scheme 26. The asymmetric ring-opening of meso-stilbene oxide with (tert-butyldimethyl(phenylselanyl)silane as nucleophile catalyzed by $\mathrm{Cr}$-salen complex $\mathbf{5 0}$.

\subsection{With Carbon-Containing Nucleophiles}

The formation of carbon-carbon bonds in one of the most fundamental and important reactions in organic synthesis. Carbon-carbon bonds form the backbone of essentially all organic molecules, 
and the asymmetric C-C bond formation is of key importance in the synthesis of optically active and highly functionalized molecules, such as biologically active compounds [88,89]. As such, the catalytic ARO of meso-epoxides with carbon-based nucleophiles represents a potentially attractive strategy for achieving this task. However, the use of metal-salen complexes as catalysts for these kinds of reactions has been limited. One example comes from Cozzi and Umani-Ronchi, who used enantiopure Cr-salen catalyst $\mathbf{1}$ (Scheme 4 ) for the ARO of meso-stilbene oxide with indoles as carbon nucleophiles, giving ring-opened products in excellent yields and enantioselectivities (Scheme 27) [90].<smiles>c1ccc([PH2+]C2OC2c2ccccc2)cc1</smiles><smiles>[R]c1cc2cc([18F])ccc2n1[R]</smiles>

$\mathrm{R}_{1}=\mathrm{H}, \mathrm{Me}$

$\mathrm{R}_{2}=\mathrm{H}, \mathrm{Me}$

$\mathrm{R}_{3}=\mathrm{H}, \mathrm{OMe}$

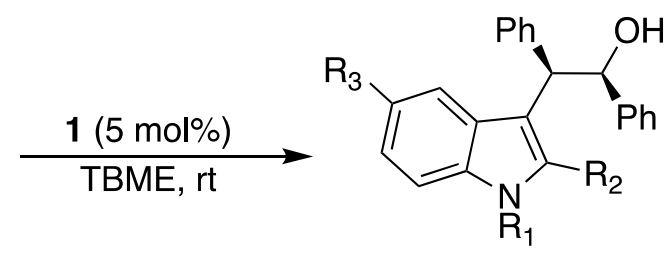

$95-98 \%(90-98 \%$ ee $)$

Scheme 27. The asymmetric ring-opening of meso-stilbene oxide with indoles catalyzed by $\mathrm{Cr}$-salen complex 1 (Scheme 4).

Pietrusiewicz and coworkers reported the use of $\mathrm{Al}(\mathrm{III})$ salen catalyst $\mathbf{5 1}$ in the ARO of 3,4-epoxy-1-phenylphospholane-1-oxide with TMSCN (Scheme 28) and $\mathrm{TMSN}_{3}$ as nucleophiles [22]. The products are potential intermediates in the synthesis of phosphasugar derivatives. It was found that the enantioselectivity was greatly influenced by the choice of solvent. For the azide addition, the highest yield was obtained in THF $(90 \%)$ and best enantioselectivity in dichloromethane ( $28 \%$ $e e)$. The use of an ionic liquid as the reaction medium gave high yields but did not increase the enantioselectivity. For the cyanide addition, the ARO of 3,4-epoxy-1-phenylphospholane-1-oxide performed with $10 \mathrm{~mol} \%$ catalyst in TBME afforded the corresponding cyanohydrin in $56 \%$ yield and $72 \%$ ee (Scheme 28).
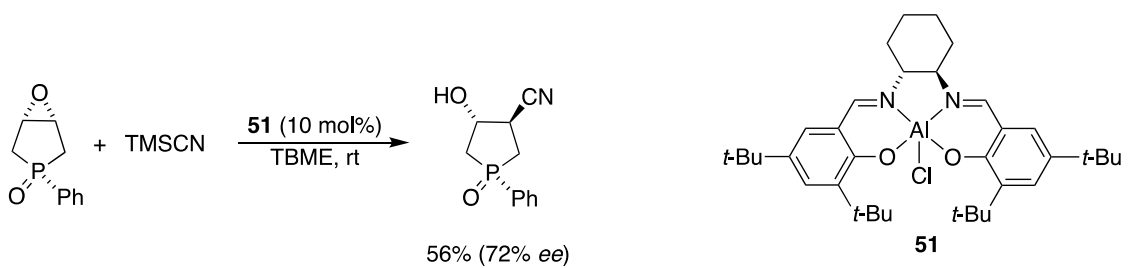

Scheme 28. The asymmetric ring-opening of 3,4-epoxy-1-phenylphospholane-1-oxide with TMSCN catalyzed by Al-salen complex 51.

Another example is the Y-salen complexes explored by RajanBabu and coworkers [23]. A number of salen complexes with different diamine backbones were employed as catalysts in the ARO of cyclohexene oxide with TMSCN. The best result in terms of enantioselectivity was obtained using binaphthyldiamine (BINAP $\left(\mathrm{NH}_{2}\right)_{2}$ ) derived salen complex 52, which gave the ring-opened product in $77 \%$ ee (Scheme 29). The catalyst loading could be reduced from $2 \mathrm{~mol} \%$ to $0.1 \mathrm{~mol} \%$ without significant loss of reactivity or enantioselectivity and the reaction could also be run under solvent-free conditions. 


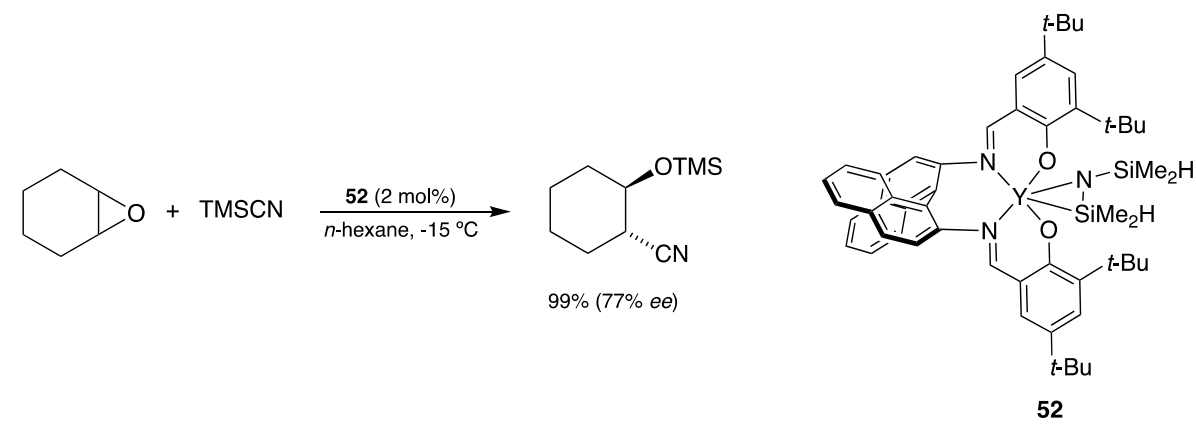

Scheme 29. The asymmetric ring-opening of cyclohexene oxide with TMSCN catalyzed by Y-salen complex 52.

\section{Kinetic Resolution of Epoxides}

Some epoxides, typically terminal ones, are difficult to prepare directly with high enantiomeric purity. Since the racemic mixtures are usually readily available at low cost, using an efficient KR becomes an attractive alternative for obtaining enantiomerically pure epoxides. Ideally, this strategy results in both the enantiopure ring-opened product, as well as the enantiomeric enrichment of the unreacted epoxide [91]. The selectivity can often be tuned towards the ring-opened product or the enantioenriched unreacted epoxide by the amount of nucleophile used.

Most of the examples presented herein concern the KR of terminal epoxides, although there are a few examples of the use of 1,2-disubstituted epoxides (mainly with trans stereochemistry) and even trisubstituted epoxides. In general, the reactions proceed with almost exclusive regioselectivity for the nucleophilic attack at the least substituted epoxide carbon.

For most of the reported examples of the KR of epoxides, the focus is on the ring-opened product. The most obvious exception is in the HKR, where the use of water as an inexpensive, environmentally friendly and highly available nucleophile makes it an attractive strategy for the enantioenrichment of the unreacted epoxide. As such, many of the references presented herein only report the yields and ee values of the product for which the method was optimized. There are also several ways of calculating the yield of the reaction. Although the theoretical maximum yield of the enantiopure ring-opened product is $50 \%$ (given that the starting material is used as a racemic mixture), many of the references calculate the yield based on the amount of nucleophile used, which varies a lot between different papers. To facilitate the comparison of different protocols, we have recalculated all yields based on the epoxide, giving a 50\% maximum yield for each product. In some references, the results are reported as conversion instead of yield. In those cases, the products are often not isolated, and the conversion is calculated based on the consumption of each of the two enantiomeric starting materials determined by chiral GC separation.

\subsection{With Azides}

Jacobsen and coworkers reported the azidolytic KR of terminal epoxides catalyzed by $\mathrm{Cr}$-salen complex 2 (Scheme 4) [92]. The reactions were run under solvent-free conditions at $0-2{ }^{\circ} \mathrm{C}$. By using 0.5 equivalents of $\mathrm{TMSN}_{3}$, the ring-opened products could be obtained in $40-49 \%$ yield and $89-97 \%$ ee . The reaction exhibited good functional group tolerance (Scheme 30). One of the investigated reactions, the azidolytic KR of epichlorohydrin, was further investigated, as it was discovered that the unreacted epoxide underwent racemization under the reaction conditions. This enabled the dynamic kinetic resolution (DKR) of epichlorohydrin, where, by adding $\mathrm{TMSN}_{3}$ in portions over time, the desired ring-opened product was obtained in 76\% yield and $97 \%$ ee (Scheme 31) [93].

The same protocol was also extended to include 2,2-disubstituted epoxides, using in situ formed $\mathrm{HN}_{3}$ (formed from TMSN 3 and isopropanol) as the nucleophile [94]. Performing the reaction in TBME afforded both ring-opened products and unreacted epoxides in high yields and enantioselectivities 
(Scheme 32). This methodology was successfully used for the enantioselective preparation of a key intermediate in a synthesis of the natural product taurospongin A [95].

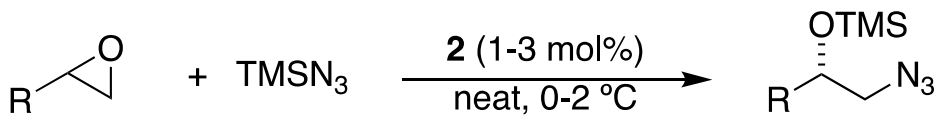

$( \pm)$

$40-49 \%(89-97 \%$ ee $)$

$$
\begin{aligned}
\mathrm{R}= & \mathrm{CH}_{3}, \mathrm{CH}_{2} \mathrm{CH}_{3}, \\
& \mathrm{CH}_{2} \mathrm{Ph}, \mathrm{CH}(\mathrm{OEt})_{2}, \\
& \mathrm{CH}_{2} \mathrm{Cl}, \mathrm{CH}_{2} \mathrm{OTBS}, \\
& \mathrm{CH}_{2} \mathrm{CN}
\end{aligned}
$$

Scheme 30. The azidolytic kinetic resolution terminal epoxides catalyzed by $\mathrm{Cr}$-salen complex 2 (Scheme 4).
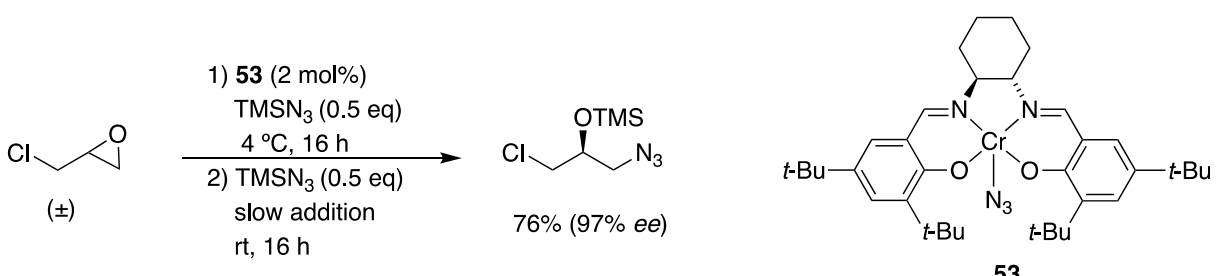

53

Scheme 31. The dynamic kinetic resolution of epichlorohydrin with $\mathrm{TMSN}_{3}$ catalyzed by $\mathrm{Cr}$-salen complex 53.

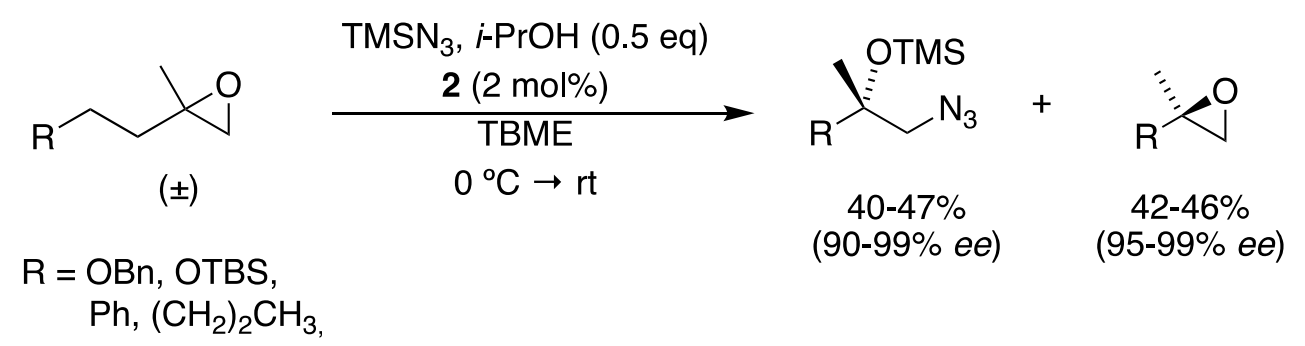

Scheme 32. The azidolytic kinetic resolution of 2,2-disubstituted epoxides catalyzed by $\mathrm{Cr}$-salen complex 2 (Scheme 4).

Jacobs and coworkers used Cr-salen complex 1 (Scheme 4) for the KR of terpene epoxides with $\mathrm{TMSN}_{3}$ [96]. This constitutes a rare example of the kinetic resolution of trisubstituted epoxides. The reaction gave enantioenriched epoxides and ring-opened products in good yields and high diastereomeric excess. Initial experiments revealed that both enantiomers of the $\mathrm{Cr}$-salen complex, $(R, R)$-complex 1 (Scheme 4 ) and $(S, S)$-complex $\mathbf{4 6}$ (Scheme 18), exhibited similar reactivity and selectivity. It was therefore hypothesized that the observed stereoselectivity of the reaction was induced by the C4 substituent (Scheme 33) and that the Cr-salen complex served only as an activating agent for the azide-transfer. As such, later experiments were performed with the $\mathrm{Cr}$-salen complex 1(46) as a racemic mixture (Scheme 33). For all investigated substrates, the cis-diastereomers were selectively transformed and the trans-epoxides remained unreacted (cis and trans refers to the relative stereochemistry of the methyl group and the $\mathrm{C} 4$ substituent). Jacobs and coworkers also investigated the effect of micro-wave irradiation on the Cr-salen-catalyzed KR of terminal epoxides, as well as the ARO of meso-epoxides. It was found that the reaction rate could be increased by up to three orders of magnitude without any significant loss of enantioselectivity under micro-wave irradiation [97]. 
<smiles>CC(C)=CCCC(C)(C)C</smiles>

\section{$+\mathrm{TMSN}_{3}$ \\ (0.7 eq)

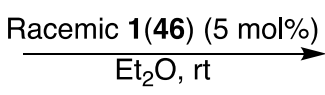<smiles>C=C(C)C1CCC2(C)CC1O2</smiles>

$43 \%(89 \%$ de $)$

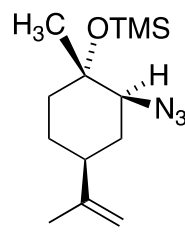

$36 \%(90 \% d e)$

Scheme 33. The kinetic resolution of (-)-limonene-1,2-epoxide with $\mathrm{TMSN}_{3}$ catalyzed by $\mathrm{Cr}$-salen complex 1(46) (Schemes 4 and 18, respectively) as a racemic mixture.

Similar to the ARO of meso-epoxides with azides, most examples of metal-salen-catalyzed KRs of epoxides with azides are based on chromium-salen complexes. One example of a different metal-salen complex was published by Kim and coworkers, who used binuclear Co(II)salen complexes bearing Lewis acids of group 13 metals (Scheme 34) [98]. The presence of a group 13 Lewis acid was necessary for catalytic activity, and the dimeric complexes showed enhanced reactivity and enantioselectivity compared to their monomeric analogues. The system allowed for low catalyst loading, where $0.5 \mathrm{~mol} \%$ of complex $\mathbf{5 4}$ catalyzed the azidolytic KR of a number of terminal epoxides, affording ring-opened products in excellent yields and with high enantioselectivity (Scheme 34).
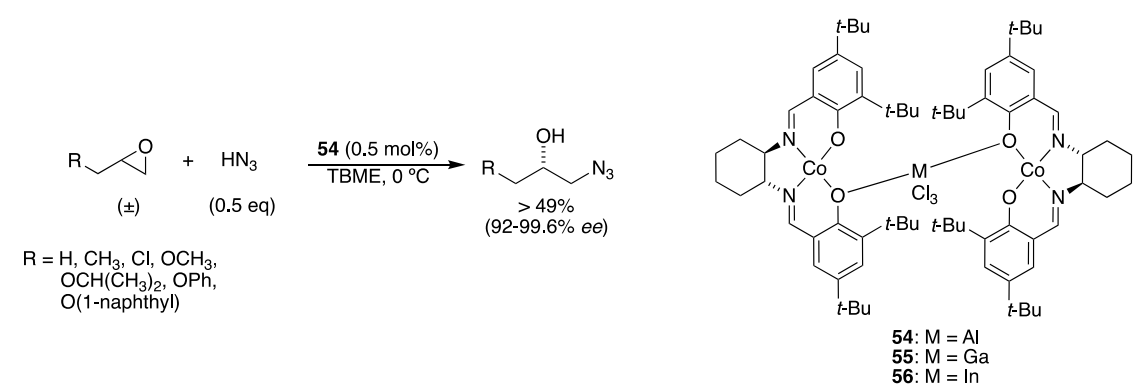

Scheme 34. The azidolytic kinetic resolution of terminal epoxides with catalyzed by dimeric Co(II)salen complexes formed by group 13 metal activation.

For the development of heterogeneous catalysts for the KR of epoxides, the same strategies as for the $\mathrm{ARO}$ of meso-epoxides have been employed. Early examples of polymer-supported $\mathrm{Cr}$-salen complexes only induced low enantioselectivities in the ring-opened products, but exhibited good stability and recyclability [40]. Jacobs and coworkers have published extensive work on the impregnation of $\mathrm{Cr}$-salen complexes on silica and silica-supported ionic liquids. Both monomeric complex $\mathbf{1}$ (Scheme 4) and dimeric complex 23 (Figure 7) were separately physiosorbed on silica and evaluated as catalysts in the KR of 1,2-epoxyhexane and 1,2-epoxyoctane, achieving quantitative conversions and high enantioselectivities (up to $98 \%$ ee for ring-opened products). The dimeric complexes showed improved enantioselectivity compared to the monomeric analogues. Using the catalyst in a continuous flow reactor decreased the deterioration of the solid support and resulted in quantitative yields and high enantioselectivities for both the ring-opened products and unreacted epoxides (up to 99 and 85\% $e e$, respectively) [44,45]. Immobilizing the dimeric complex in a silica-supported ionic liquid further improved the reactivity and enantioselectivity, and both the catalyst and the ionic liquid could be recovered by Soxhlet extraction with acetone [46].

Liu and coworkers investigated the macrocyclic oligomer-supported Cr-salen catalysts 29-31 (Figure 10) in the KR of 1,2-epoxyhexane and propylene oxide [49]. The same trend as in the ARO of meso-epoxides was found, where a shorter linker between the $\mathrm{Cr}$-salen complex and the macrocycle increased the reaction rate and gave the highest yield (45-48\%), while a longer linker gave better enantioselectivity $(83-84 \%$ ee) for the ring-opened product.

Yang and Li constructed an efficient solid nanoreactor by encapsulating $\mathrm{Cr}$-salen complex $\mathbf{1}$ (Scheme 4) and pyridine inside a mesoporous silica nanocage [99]. The addition of pyridine led 
to a pronounced increase in TOF and enantioselectivity, which was attributed to the increased nucleophilicity of the $\mathrm{Cr}$-salen complex after coordination to pyridine. The system was investigated in the KR of 1,2-epoxyhexane with $\mathrm{TMSN}_{3}$ under solvent-free conditions and at very low catalyst loading $(0.002 \mathrm{~mol} \%)$, affording both ring-opened product and unreacted epoxide in close to quantitative yield and with high enantioselectivity ( $91 \%$ ee and $92 \%$ ee respectively). The nanoreactor showed high stability and could be recovered and recycled nine times with maintained reactivity and enantioselectivity.

\subsection{With Anilines, Amines and Carbamates}

Bartoli and Melchiorre investigated different metal-salen catalysts for the KR of epoxides with different nitrogen-based nucleophiles. Cr-salen complex $\mathbf{1}$ (Scheme 4) was used for the KR of a variety of trans-disubstituted aromatic epoxides with aniline and anisidine (Scheme 35). Complete regioand diastereoselectivity was observed, and the vicinal anti-amino alcohol products were obtained in reasonable yields and enantioselectivities $(77-99 \% e e)$. The study also included a rare example of the $\mathrm{KR}$ of a trisubstituted epoxide, where applying the protocol on trans-2-methyl-2,3-diphenyl oxirane afforded the ring-opened products in $18 \%$ yield and $81 \%$ ee [54].

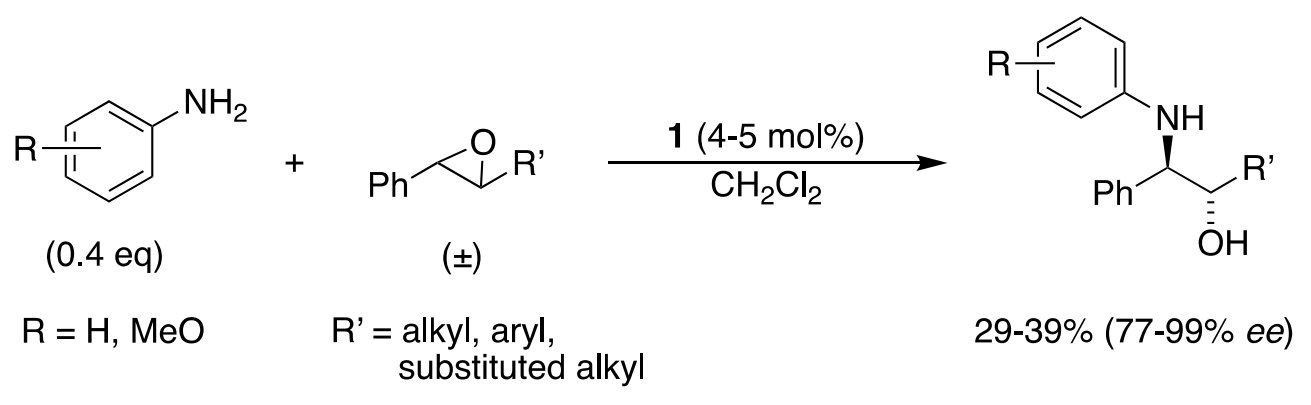

Scheme 35. The aminolytic kinetic resolution of internal trans-epoxides catalyzed by $\mathrm{Cr}$-salen complex 1

(Scheme 4).

The same group also investigated the KR of terminal epoxides using tert-butyl arbamate as the nucleophile. Co(II)salen complex 45 (Figure 20) was used as a pre-catalyst and oxidized in situ to the corresponding $\mathrm{Co}(\mathrm{III})$ salen complex by addition of $p$-nitrobenzoic acid, affording enantiopure $(e e \geq 99 \%) \mathrm{N}$-protected vicinal amino alcohols in high yields and with complete regioselectivity, while the unreacted epoxides could also be isolated with high ee values (Scheme 36). The protocol was effective for both linear and relatively hindered aliphatic epoxides, as well as epoxides containing different functional groups [100].

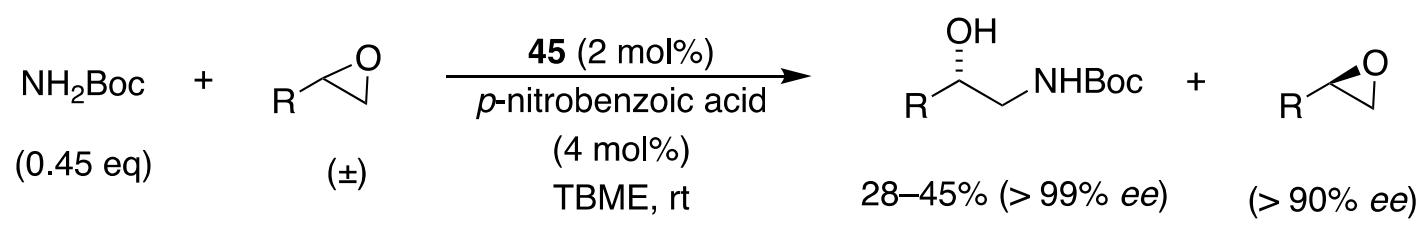

Scheme 36. The carbamolytic kinetic resolution of terminal epoxides employing Co(II)salen complex 45 as a pre-catalyst (Figure 20).

The use of ionic liquids as the reaction medium is considered an environmentally friendly alternative to traditional organic solvents [101]. This was exploited by Kureshy and coworkers in several studies on the KR of epoxides. In one such study, the protocol presented in Scheme 36 was further improved by the use of ionic liquids as reaction medium [102]. Performing the reaction in [bmim] $\left[\mathrm{PF}_{6}\right]$ afforded both ring-opened products and unreacted epoxides in excellent yields and enantioselectivities for a number of glycidyl ethers and terminal alkyl epoxides (Scheme 37). The reactions were completed in $5-10 \mathrm{~h}$ and the catalyst and ionic liquid could be recycled six times with maintained enantioselectivity 
and reactivity. The choice of nucleophile could be extended to urethane and benzyl carbamate with equally excellent yield and enantioselectivity.
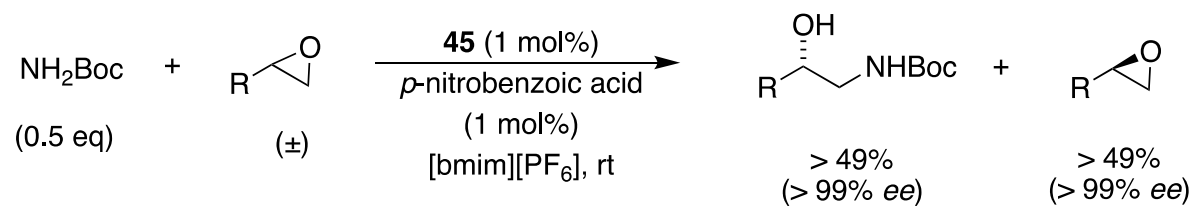

Scheme 37. The carbamolytic kinetic resolution of terminal epoxides in ionic liquid, employing Co(II)salen complex 45 (Figure 20) as the pre-catalyst.

In another study, Kureshy and coworkers investigated the KR of trans-stilbene oxide and trans- $\beta$-methyl styrene oxide with different anilines catalyzed by $\mathrm{Cr}$-salen complex 53 (Scheme 31) [103]. Performing the reaction in [bmim] $\left[\mathrm{PF}_{6}\right]$ (Figure 11) afforded ring-opened products in $40-49 \%$ yield and $63-99 \%$ ee. The unreacted epoxides could be recovered in quantitative yield and in $60-97 \%$ ee. The use of ionic liquids had several advantages, including easy product separation (extraction with $n$-hexane), efficient recyclability of the catalyst and the ionic liquid, and significantly reduced reaction times compared to reactions run in conventional organic solvent.

In another effort to improve the recyclability of the catalyst, Kureshy and coworkers employed the enantiomerically pure polymeric $\mathrm{Cr}$-salen complex 57 (Figure 22) in the KR of trans-stilbene oxide and trans- $\beta$-methyl styrene oxide with anilines as nucleophiles [104]. The reactions were performed in dichloromethane. The desired vicinal amino alcohols were obtained in good to excellent yield (35-49\%) and high enantiomeric excess (up to 100\% ee after a single recrystallization). The unreacted epoxide could be recovered in excellent yield (48-51\%) and good to high enantiomeric excess (70-98\% $e e$ ). The catalyst could be easily recovered by precipitation with $n$-hexane and recycled up to four times with maintained enantioselectivity. In a separate study, the same reaction was carried out with dimeric and polymeric $\mathrm{Cr}$-salen complexes under microwave irradiation in a $\mathrm{CH}_{2} \mathrm{Cl}_{2} / \mathrm{MeOH}$ mixture (1:1), which allowed for very short reaction times (2 $\mathrm{min}$ ) [105]. The best results were achieved with dimeric complex 23 (Figure 7) in the KR of trans-stilbene oxide with different anilines. The ring-opened products were obtained in $45-49 \%$ yield and $88-94 \%$ ee and the unreacted epoxides were isolated in $47-48 \%$ yield and $80-92 \%$ ee. Both dimeric catalyst 23 and polymeric catalyst 57 could be recycled five times without loss of reactivity or enantioselectivity.

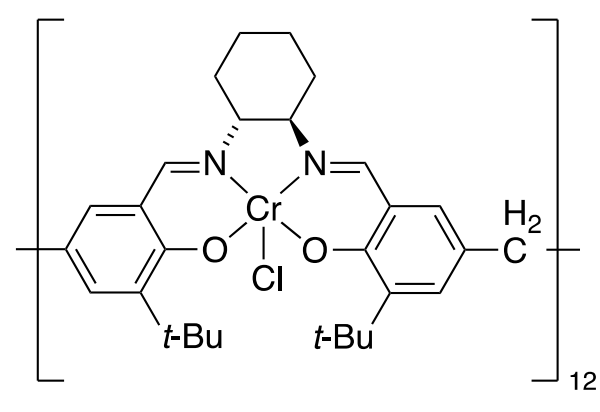

57

Figure 22. Polymeric Cr-salen complex 57.

The same group also developed a series of $\mathrm{Cr}$-salen complexes with cationic side groups (catalyst 58-60, Figure 23), which were used in the KR of trans-epoxides with different anilines [106]. The best results were obtained with catalyst 60 (Figure 23) in the KR of trans-stilbene oxide in dichloromethane, where the ring-opened products were obtained in excellent yields (41-49\%) and with high enantioselectivities $(86-99 \% e e)$ and the unreacted epoxides were recovered in quantitative 
yield and $89-99 \%$ ee. The catalyst could be recycled up to six times without loss of reactivity or enantioselectivity.

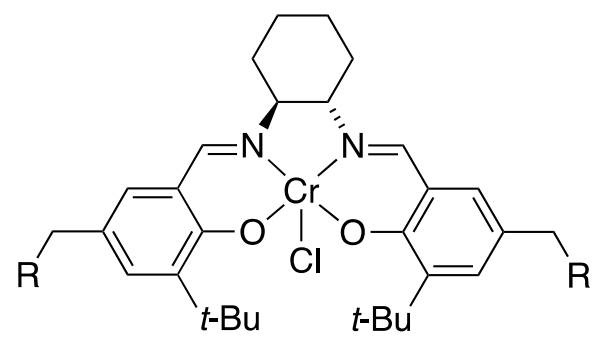

58: $\mathrm{R}=\mathrm{PPh}_{3}{ }^{+} \mathrm{Cl}^{-}$

59: $\mathrm{R}=\mathrm{NEt}_{3}{ }^{+} \mathrm{Cl}^{-}$

60: $\mathrm{R}=\mathrm{C}_{5} \mathrm{H}_{5} \mathrm{~N}^{+} \mathrm{Cl}^{-}$

Figure 23. Cr-salen complexes with cationic side chains.

Kureshy and coworkers investigated macrocyclic Cr-salen complex 35 (Figure 14) in the KR of trans-epoxides with anilines The ring-opened products were obtained in excellent yields (46-49\%) and with high enantioselectivities (up to $>99 \% e e$ ), with concomitant recovery of the unreacted epoxides in quantitative yields and with high enantioselectivities (up to $>99 \%$ ee) [56]. The reactions were performed in a $\mathrm{CH}_{2} \mathrm{Cl}_{2} / \mathrm{MeOH}$ mixture and the catalyst showed excellent recyclability. The same group also developed another set of macrocyclic $\mathrm{Cr}$-salen complexes [107]. Complex 61 (Figure 24) was used as a catalyst for the synthesis of pharmaceutically important $\beta$-amino- $\alpha$-hydroxyl ester derivatives by the KR of aromatic ester epoxides with anilines. The ring-opened products were obtained in high yields and with high diastereoselectivities and enantioselectivities (Scheme 38). The catalyst showed good stability and good recyclability.

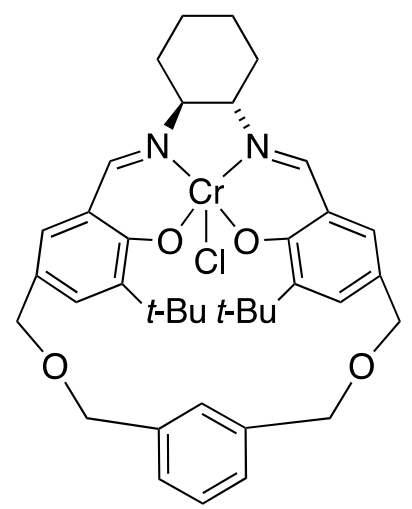

61

Figure 24. Macrocyclic Cr-salen complex 61.

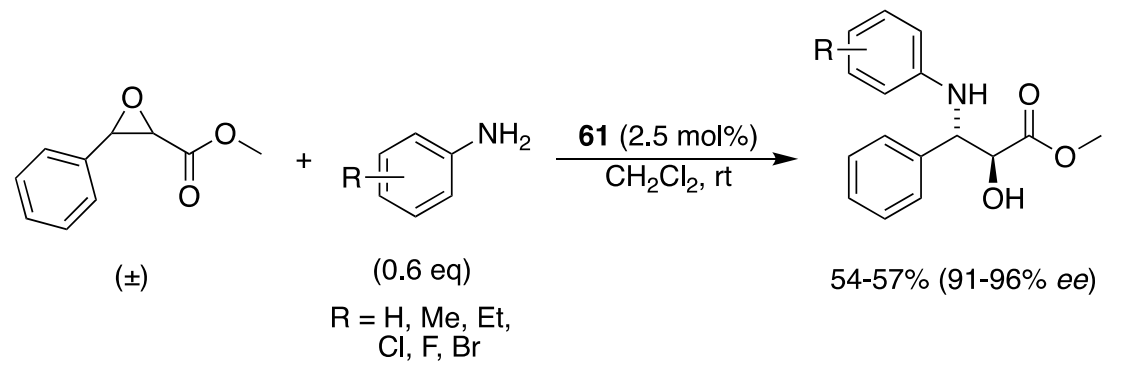

Scheme 38. The aminolytic kinetic resolution of aromatic ester epoxides catalyzed by macrocyclic Cr-salen complex 61 (Figure 24). 
For the KR of epoxides with carbamates as nucleophiles, Kureshy and coworkers developed a number of chiral polymeric Co-salen complexes with chiral and achiral linkers. The complexes were evaluated as catalysts in the KR of a number of glycidyl ethers and terminal alkyl epoxides with different carbamates [108]. The reaction with $1 \mathrm{~mol} \%$ of catalyst 62 (Figure 25) in dichloromethane afforded both epoxides and $\mathrm{N}$-protected vicinal amino alcohols in quantitative yield and with excellent enantioselectivity $(>99 \%$ ee) in $16 \mathrm{~h}$. The catalyst could be precipitated with $n$-hexane and reused up to six times with complete retention of enantioselectivity.

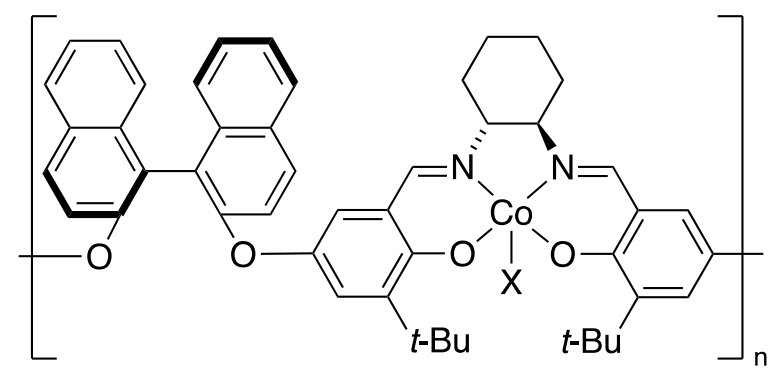

$62(\mathrm{X}=p$-nitrobenzoic acid $)$

Figure 25. Polymeric Co-salen complex 62.

Jacobsen and coworkers showed that their oligomeric Co-salen complex 35 (Scheme 10) could be used in the KR of 1,2-epoxyhexane with tert-butyl carbamate, where a low catalyst loading $(0.2 \mathrm{~mol} \%)$ was enough to obtain the ring-opened product in high yield and enantioselectivity (Scheme 39) [58].

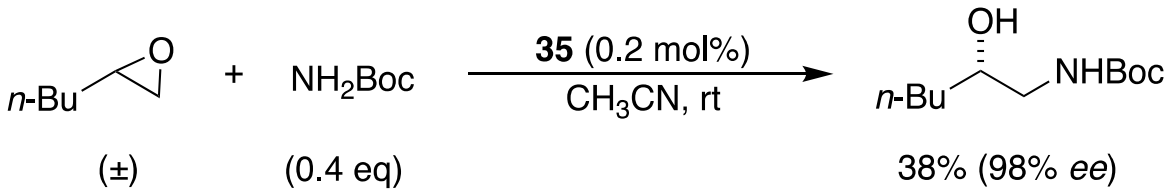

Scheme 39. The carbamolytic kinetic resolution of 1,2-epoxyhexane catalyzed by oligomeric Co-salen complex 35 (Scheme 10).

\subsection{With Water (Hydrolytic Kinetic Resolution)}

Since the first report by Jacobsen in 1997, the hydrolytic kinetic resolution (HKR) of terminal epoxides has been one of the most researched applications of metal-salen catalysts. The use of water as an inexpensive and environmentally friendly nucleophile also makes it an attractive method for the enantioenrichment of epoxides otherwise difficult to obtain. The protocols have mainly used $\mathrm{Co}(\mathrm{III})$ salen catalysts and the reactions are characterized by excellent yields and enantioselectivities. Hence, instead of only focusing on obtaining high yields and enantioselectivity, much effort has been focused on increasing cooperativity and reaction kinetics, decreasing catalyst loading, as well as developing heterogeneous systems and new catalytic methodologies in general.

In their original paper, Jacobsen and coworkers found that Co(III)salen complex 63 (Scheme 40), formed in situ from the corresponding Co(II)salen complex, worked very well in the HKR of several terminal alkyl, alkenyl, and aryl epoxides, resulting in unreacted (S)-terminal epoxides and (R)-1,2-diols with excellent yields (up to $46 \%$ and 50\%, respectively) and ee values (up to $99 \%$ and $98 \%$, respectively) [109]. The catalyst could be easily regenerated by treatment with acetic acid in air and reused in two cycles without loss of reactivity or enantioselectivity. Later, the scope was extended to a broad group of epoxides with different steric and electronic environments [110]. In almost all cases, the unreacted epoxides could be obtained in $>99 \%$ ee (Scheme 40a). Furthermore, by tuning the equivalents of water and catalyst loading, most of the 1,2-diols could be obtained with excellent enantioselectivities (94-99\% ee) (Scheme 40b). 


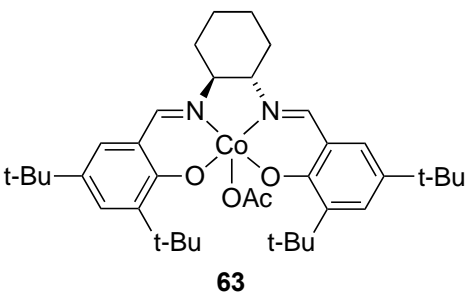

(a)

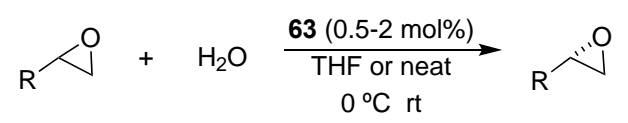

$( \pm)$

(0.55-0.70 eq)

$36-48 \%$

(98-99\% ee)

(b)

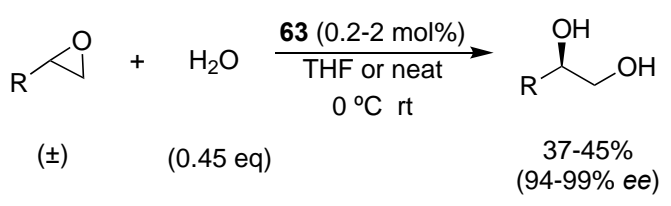

Scheme 40. The hydrolytic kinetic resolution of terminal epoxides catalyzed by Co-salen complex

63. The displayed reaction conditions are optimized for enantioenrichment of (a) (S)-epoxides;

(b) (R)-1,2-diols.

For cases where the diol was required in very high enantiomeric excess, a strategy called "double resolution" was suggested and employed in the synthesis of (S)-3-chloropropane-1,2-diol (Scheme 41). Epichlorohydrin was first subjected to HKR with $(R, R)$-salen complex 64 as the catalyst (Figure 26). The enantioenriched unreacted epoxide was then separated and subjected to another HKR with the opposite enantiomer of the catalyst, $(S, S)$-salen complex 63 (Scheme 40), which resulted in enantiopure (S)-3-chloropropane-1,2-diol in $41 \%$ overall yield.

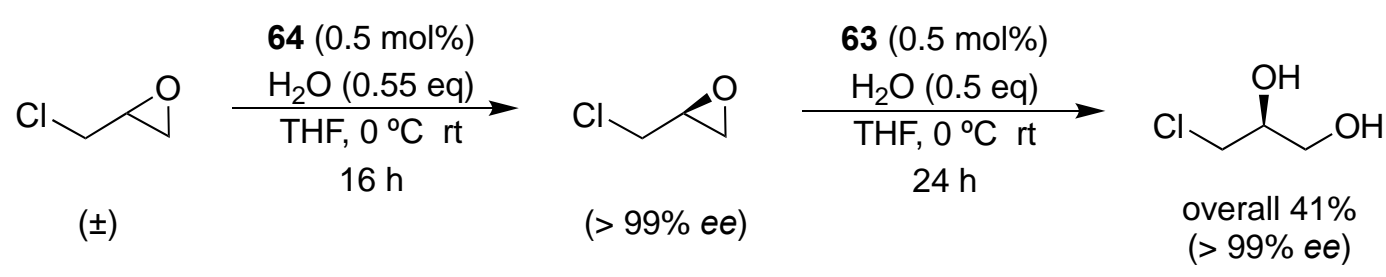

Scheme 41. Preparation of enantiopure (S)-3-chloropropane-1,2-diol using a "double resolution" strategy employing catalyst 63 (Scheme 40) and 64 (Figure 26).

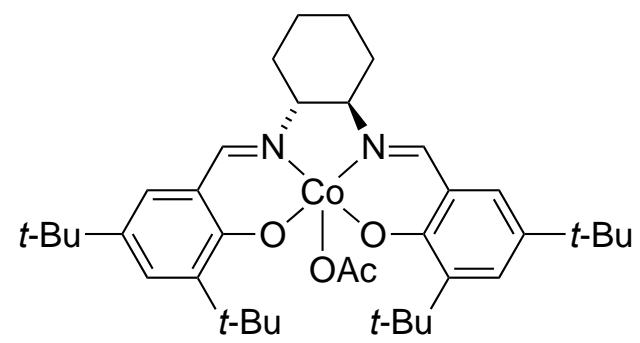

64

Figure 26. Co-salen complex 64.

The high reactivity and excellent enantioselectivity of complex 63 and 64 in the HKR of a broad range of terminal epoxides set a high standard for catalysts for these reactions. Subsequent studies have largely focused on improving other aspects of the catalysis, including reducing reaction times (average reaction time for catalyst 63 was $12-18 \mathrm{~h}$, sometimes up to $72 \mathrm{~h}$ ), reducing catalyst loading and enabling solvent-free reactions. Another important issue is catalyst recycling. For instance, homogenous catalyst 63 was recycled as a solid residue by distilling off the diols and unreacted epoxides during work up, a method which is time-consuming and only applicable for sufficiently volatile products. In addition, catalyst 63 needed to be regenerated with acetic acid in air before it could be recycled. 


\subsubsection{Multi-Metallic Catalysts}

Similar to what was observed for the azidolytic ARO of meso-epoxides catalyzed by $\mathrm{Cr}$-salen complexes, preliminary kinetic studies indicated a second-order dependence on catalyst 63 in the HKR of terminal epoxides [109]. Consequently, a similar cooperative bimetallic pathway was proposed as a possible mechanism for the HKR of epoxides (Figure 1). Since then, numerous studies have reported multi-metallic catalysts where several salen moieties are linked together in order to facilitate this bimetallic pathway. Larger systems also have the advantage of often being less soluble and therefore more easily recyclable.

Kureshy and coworkers [111,112] synthesized dimeric Co-complexes 65 and 66 (Figure 27). Two Co(III)salen moieties were covalently linked by a methylene or propane-2,2-diyl group, with the expectation that the increased molecular weight would result in an easier catalyst recovery compared to the monosalen complexes. This very straight-forward way of linking two salen moieties together was also employed by Jacobs and coworkers [44] in their design of dimeric Cr-salen complex 23 (Figure 7), which was impregnated on silica and used as a heterogeneous catalyst for the azidolytic ring-opening of meso-epoxides. Complex 65 was employed in the HKR of epichlorohydrin, propylene oxide and styrene oxide under solvent-free conditions. Complex 66 was investigated in the HKR of epichlorohydrin and a variety of alkyl terminal epoxides. For all substrates, the ring-opened diols and the unreacted epoxides were obtained with excellent yields (up to $50 \%$ for the diols and up to $52 \%$ for the epoxides) and enantiomeric excesses (up to $99 \%$ for both the diols and the epoxides). In addition, the dimeric complexes could be recycled and reused for up to four cycles without significant loss of reactivity or enantioselectivity $[111,112]$.

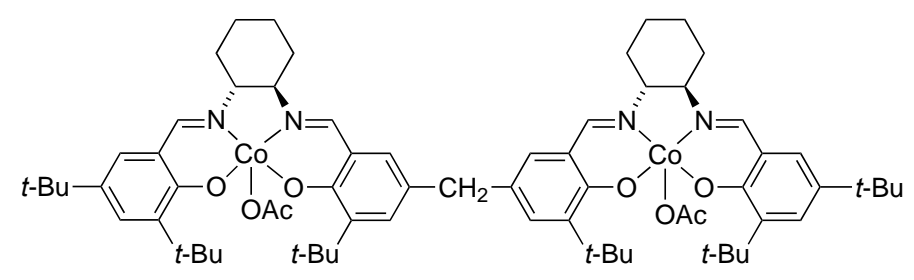

65

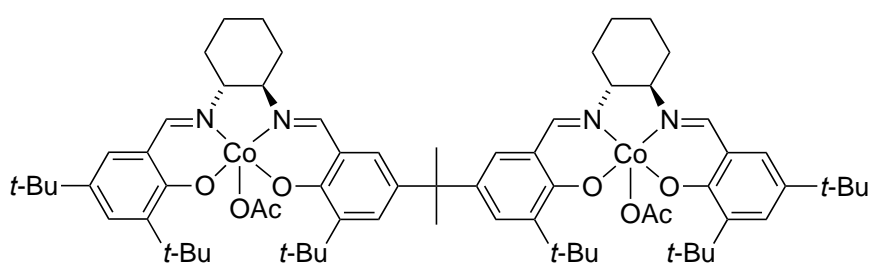

66

Figure 27. Dimeric Co(III)salen complexes 65 and 66.

In an effort to design versatile and easily recyclable Co-salen complexes capable of cooperative catalysis, Jones and coworkers synthesized styryl-substituted bissalen complex 67 , which could be polymerized to give access to homogeneous catalysts 68 and 69 and heterogeneous catalyst 70 (Figure 28) [113]. All the complexes were investigated as catalysts for the HKR of epichlorohydrin under solvent-free conditions, with reaction conditions optimized for the enantioenrichment of the unreacted epoxide. For homogenous catalysts $67-69$, full conversion of one of the enantiomers $(50 \%)$ was achieved in $7 \mathrm{~h}$ with only $0.02 \mathrm{~mol} \%$ Co loading, affording highly enantioenriched unreacted epoxide $(99 \%$ ee). As a comparison, the corresponding monosalen catalyst 64 (Figure 26) afforded the unreacted epoxide in $13 \%$ ee under the same reaction conditions and reaction time. The increased enantioselectivity and reactivity was attributed to the facilitated cooperative pathway with the dimeric catalyst. For the heterogenous catalyst 70, a slightly higher catalyst loading $(0.04 \mathrm{~mol} \% \mathrm{Co})$ was needed. At this catalyst loading, catalyst $\mathbf{7 0}$ achieved full conversion of one of the enantiomers 
of the epoxide and $99 \%$ ee for the unreacted epoxide of the opposite absolute configuration in $5 \mathrm{~h}$. Furthermore, the insoluble catalyst $\mathbf{7 0}$ could be recovered by simple filtration, then regenerated and reused. The enantioselectivity of catalyst $\mathbf{7 0}$ was retained over three cycles, although each cycle required longer reaction time to reach full conversion.

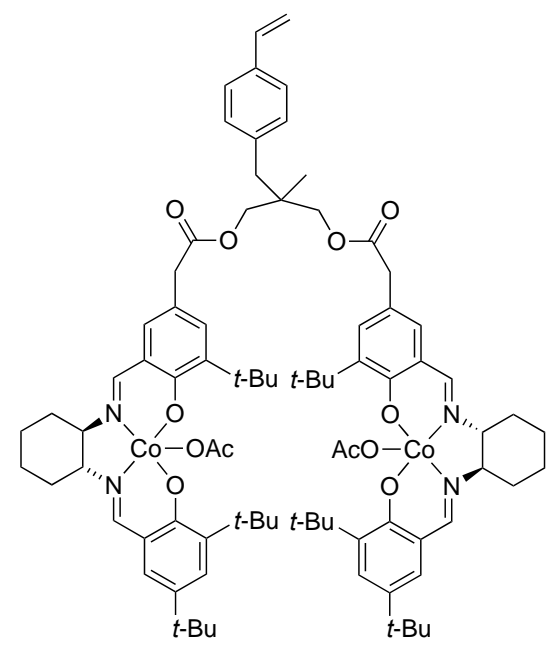

67

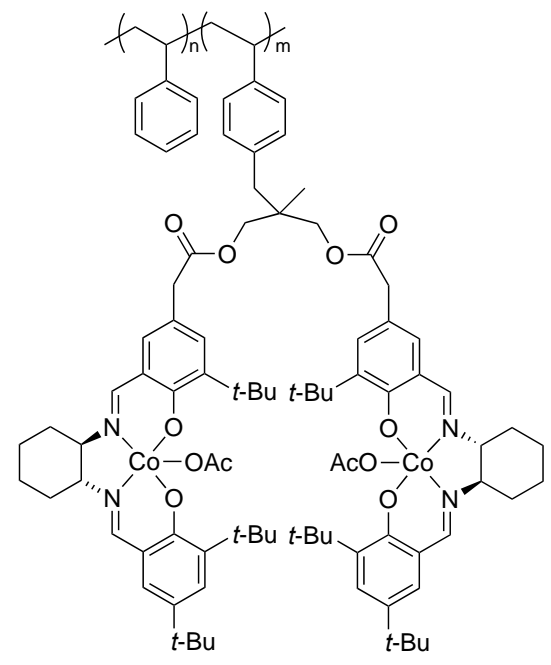

68: $m: n=1: 4$

69: $m: n=1: 12$

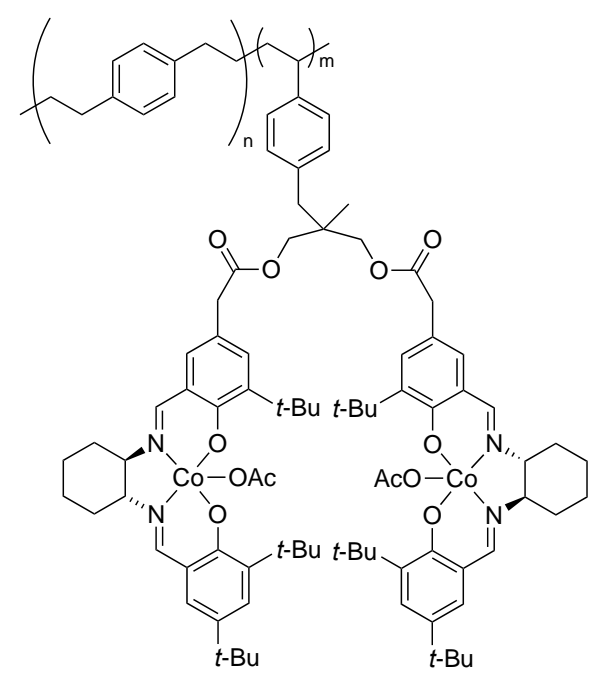

70: $m: n=1: 10$

Figure 28. Dimeric Co-salen complex 67 and polymeric complexes 68-70.

Calix[4]arene-based dimeric Co(III)salen complex 71 (Figure 29) was reported by Wezenberg and Kleij [114]. Two salen moieties were installed on the upper rim of a calix[4]arene, with the expectation of facilitating the cooperative pathway in the HKR of terminal epoxides. Catalyst $\mathbf{7 1}$ and monosalen analogues $\mathbf{7 2}$ and $\mathbf{7 3}$ (Figure 29) were separately employed in the HKR of 1,2-epoxyhexane, epichlorohydrin and styrene oxide in acetonitrile, with conditions optimized for the production of the enantioenriched epoxide. All catalysts gave comparable conversions (32-52\%), while catalyst 71 gave slightly lower ee values of both the ring-opened product and the unreacted epoxide (83-91\% and $83-97 \%$, respectively) than monosalen analogues 72 and 73 (88-95\% and $97-99 \%$, respectively). Kinetic studies showed that the HKR reactions employing catalyst $\mathbf{7 1}$ predominantly followed an intramolecular cooperative pathway. Comparison of the intra- and intermolecular rate constants for $\mathbf{7 1}$ and $\mathbf{7 2}$ revealed that although $\mathbf{7 1}$ had a significant intramolecular rate constant, as expected, 
the intermolecular rate constant was lower than for monosalen analogue 72. This is remarkable since many other bimetallic salen catalysts show both increased intra- and intermolecular rate constants in the HKR reaction compared to the corresponding monosalen catalysts [21]. For catalyst $\mathbf{7 1}$, the lower intermolecular rate constant meant that at higher catalysts loadings, the overall initial rate was lower than for the monosalen analogue $\mathbf{7 2}$, while at lower catalyst loadings the intermolecular pathway was suppressed and catalyst $\mathbf{7 1}$ showed higher reaction rates. The authors suggest that the decreased intermolecular reaction rate could be caused by the way the salen moieties were immobilized on the calixarene [114].

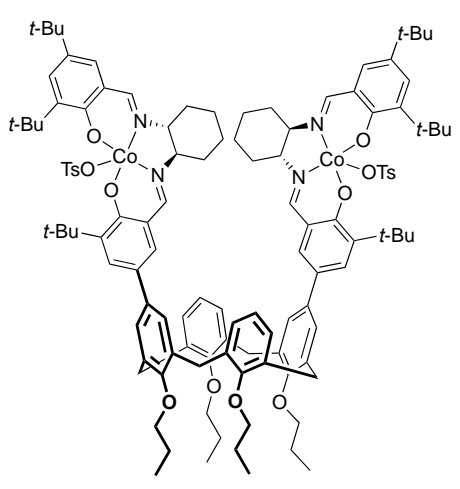

71

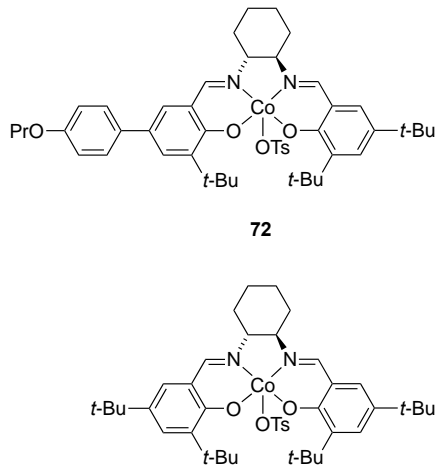

73

Figure 29. Calix[4]arene-based dimeric Co-salen complex 71 and monomeric analogues 72 and 73.

Jacobsen and coworkers $[58,69,115]$ developed three generations of oligomeric Co(III)salen complexes. Apart from catalyzing the ARO of meso-epoxides with water and carbamate as nucleophiles, the first generation (catalyst 40, Figure 18) also catalyzed the HKR of terminal epoxides [69]. With enantioselectivity similar to monosalen complex $\mathbf{6 4}$ (Figure 26), catalyst $\mathbf{4 0}$ allowed a 10- to 50 -fold decrease in catalyst loading $(\mathrm{mol} \% \mathrm{Co})$ and up to 16 -fold decrease in reaction time compared to catalyst 64 (Table 1). For the second generation of catalysts (complex 74 and 75 in Figure 30), the major difference was the linker. The use of a pimelate tether gave an oligomeric complex with a more predictable and reliable conformation [115]. Both complexes worked very well in the HKR of terminal epoxides, and the catalyst loading and reaction time were further decreased compared to the first generation (Table 1). The third generation (catalyst 35, Scheme 10) exhibited better solubility and could be synthesized with high overall yield on a large scale [58]. The catalyst loading in the HKR of terminal epoxides could be reduced down to $0.003 \mathrm{~mol} \%$ Co while retaining excellent enantioselectivity. Furthermore, its lipophilicity made catalyst 35 a good catalyst for solvent-free reactions (Table 1).

Table 1. Comparison of catalytic properties of oligosalen Co(III) complexes in the hydrolytic kinetic resolution (HKR) of terminal epoxides.

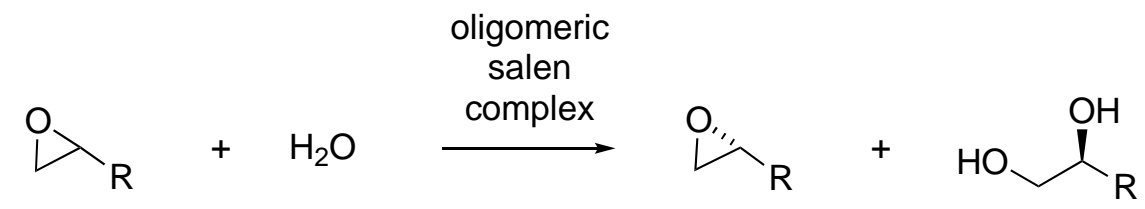

$( \pm)$

\begin{tabular}{|c|c|c|c|c|}
\hline Catalyst & Lowest $\mathrm{mol} \%$ Co & Epoxide & Diol & Solvent \\
\hline 63 or 64 & 0.5 & $36-48 \%(98-99 \%$ ee) & $37-45 \%(94-99 \%$ еe) & solvent-free or THF \\
\hline 40 & 0.01 & $36-45 \%(99 \% е e)$ & $40-50 \%(94-98 \%$ ee $)$ & solvent-free \\
\hline 74 or 75 & 0.0004 & $44-45 \%(>99 \%$ eе $)$ & $44-51(97 \%$ ee $)$ & $\mathrm{MeCN}$ or $\mathrm{MeCN} / \mathrm{CH}_{2} \mathrm{Cl}_{2}$ \\
\hline 35 & 0.0003 & $35-44 \%(>99 \%$ ee $)$ & - & solvent-free \\
\hline
\end{tabular}




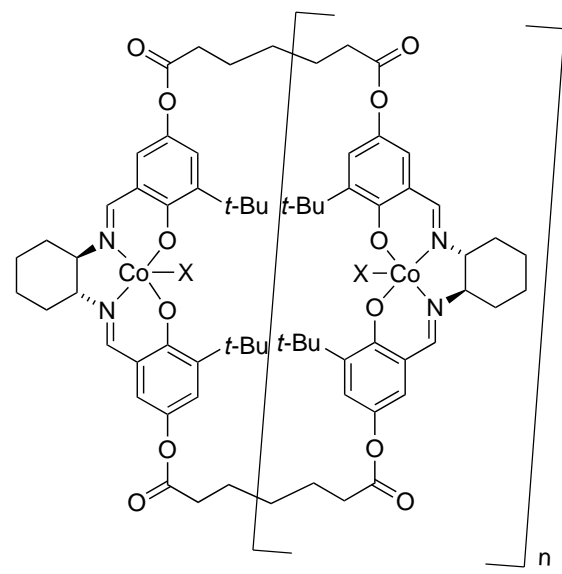

74: $\mathrm{X}=(1 S)-(+)-10$-camphorsulfonate, $\mathrm{n}=1-3$ 75: $\mathrm{X}=3-\mathrm{NO}_{2}-\mathrm{C}_{6} \mathrm{H}_{4} \mathrm{SO}_{3}, \mathrm{n}=1-3$

Figure 30. Jacobsen and coworkers' [115] second generation of oligosalen catalysts 74 and 75 .

Besides Jacobsen, Weck, and coworkers [48] also developed macrocyclic oligomers. They reported macrocyclic $\mathrm{Co}(\mathrm{III})$ salen oligomers 76 and 77 (Figure 31). Unlike other macrocyclic oligosalen complexes where the salen was part of the macrocycle, oligomers $\mathbf{7 6}$ and 77 consisted of a central macrocyclic backbone onto which the salen moieties were connected in a pendant-like fashion. This added flexibility afforded excellent reactivity and enantioselectivity in the HKR of terminal epoxides with very low catalyst loadings (down to $0.01 \mathrm{~mol} \% \mathrm{Co}$ ). For a number of terminal alkyl epoxides and glycidyl ethers, the unreacted epoxides could be obtained in up to $48 \%$ yield and $>99 \%$ ee under solvent-free conditions. More sterically hindered epoxides such as styrene oxide and tert-butyloxirane could also be resolved with similar reactivities and enantioselectivities, but higher catalyst loading $(0.1-0.25 \mathrm{~mol} \% \mathrm{Co})$ and longer reaction times were required. Interestingly, a linear polymeric analogue 78 (Figure 31) gave poorer results than macrocyclic catalysts $\mathbf{7 6}$ and $\mathbf{7 7}$ in terms of both reactivity and enantioselectivity, which reinforced the important role of the macrocyclic structure for this class of catalysts. A later study from the same group described the copolymerized cross-linked macrocyclic Co-oligosalen mixture $\mathbf{7 9}$ (Figure 32). With similar catalytic properties to 76 and $\mathbf{7 7}$, oligomeric mixture $\mathbf{7 9}$ was more synthetically available and, therefore, more practical for larger scale catalysis [116].

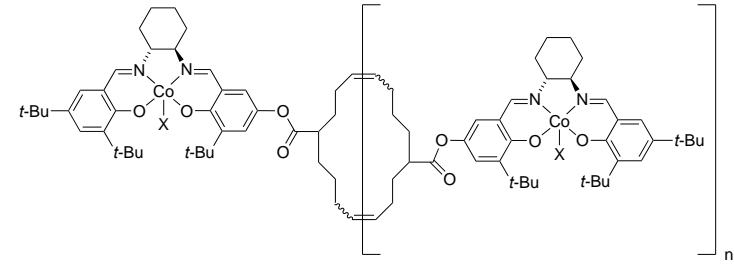

76: $\mathrm{X}=\mathrm{OAc}, \mathrm{n}=1-9$

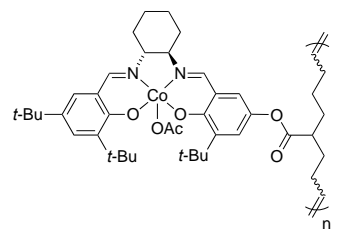

78: $n=48$

Figure 31. Macrocyclic oligosalen catalysts 76 and 77, and linear polymeric analogue 78.

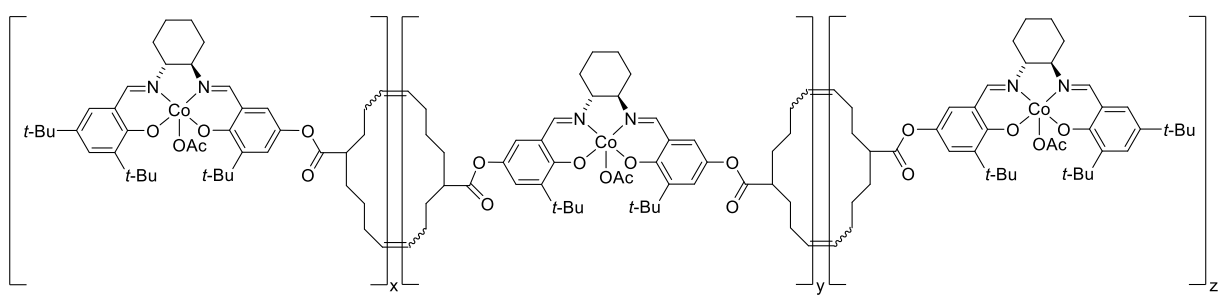

79

Figure 32. Cross-linked macrocyclic oligosalen catalyst 79. 
Khan and coworkers [117] designed a group of cyclic bissalen complexes where two Co-salen moieties were tethered by ethylene glycol chains (80-84 in Figure 33). Co-complexes 80, 81, and 84 catalyzed the HKR of a number of terminal epoxides and glycidyl ethers with excellent yields (46-53\% for the so formed 1,2-diols and $43-47 \%$ for the unreacted epoxides) and enantioselectivities (92-96\% $e e$ for the 1,2-diols and $96-99 \%$ ee for the unreacted epoxides) under solvent-free conditions and at low catalyst loadings (down to $0.016 \mathrm{~mol} \% \mathrm{Co}$ ). Catalyst 84 could be recycled up to three times with maintained reactivity and enantioselectivity and could be used directly without catalyst regeneration between cycles. For the HKR of epichlorohydrin, catalyst 84 maintained its performance on a multigram scale, illustrating the scalability of the protocol. (S)-Propylene oxide obtained from this methodology was applied in a short synthesis of $(R)$-mexiletine with high overall yield $(80 \%)$ and $e e(98 \%)$.

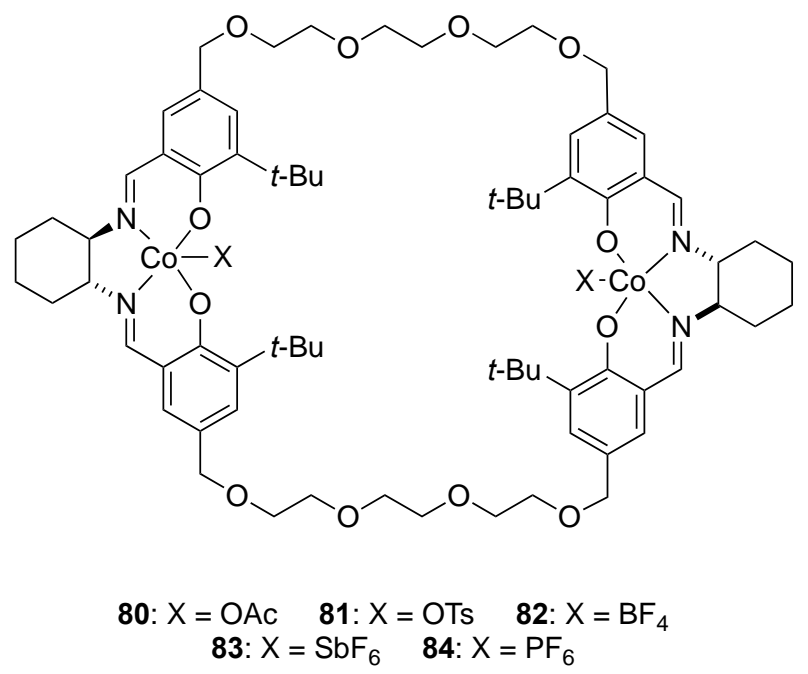

Figure 33. Cyclic bissalen complexes 80-84 containing ethylene glycol chains. The counterions $(X)$ are formally illustrated as bound to the metal.

Kureshy and Ganguly [118] reported a series of polymeric catalysts where the Co-salen moieties were tethered by different chiral and achiral linkers. Preliminary studies of the HKR of 1,2-epoxyhexane revealed that the highest enantioselectivity was obtained with polymeric $\mathrm{Co}$-salen complexes with chiral BINOL linkers where the absolute configuration of the BINOL linker and the salen ligand were opposite (i.e., $(S, R, R)$ in catalyst 85 and $(R, S, S)$ in catalyst 86, Figure 34$)$. The preferred enantiomeric pairing of the two components was supported by DFT calculations comparing $(R, S, S)$ catalyst with $(S, S, S)$ catalyst. When employed in the HKR of a broad range of terminal alkyl and aryl epoxides and glycidyl ethers, catalyst 86 enabled excellent yields and enantioselectivities of both the ring-opened products and the unreacted epoxides (Scheme 42). Catalyst $\mathbf{8 6}$ could be recycled six times in the HKR of 1,2-epoxyhexane without loss of enantioselectivity, and only required one catalyst regeneration (between cycle 4 and 5). To show the scalability of the protocol, catalyst $\mathbf{8 6}$ was successfully employed in gram-scale syntheses of chiral $\beta$-blockers (S)-metoprolol, (S)-toliprolol and (S)-alprenol, where the final products were obtained in moderate overall yields (up to $44 \%$ ) and excellent enantioselectivities (>99\%).

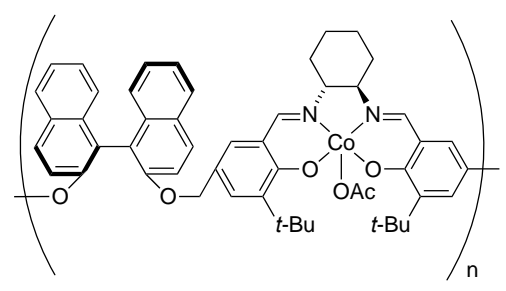

85

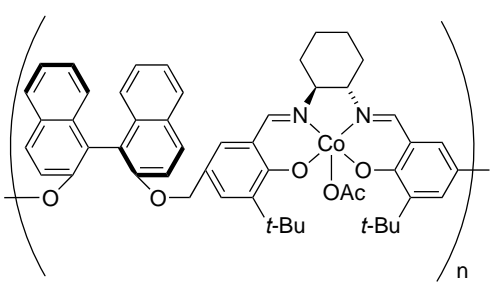

86

Figure 34. Polymeric Co-salen catalysts with 1,1'-bi-2-naphthol (BINOL) linkers. 


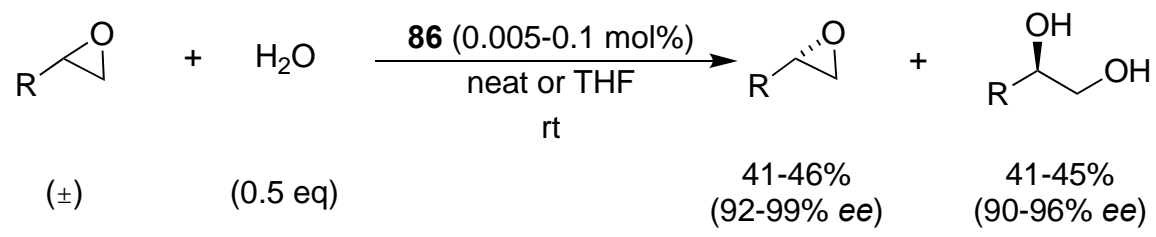

Scheme 42. The hydrolytic kinetic resolution of terminal epoxides catalyzed by catalyst 86 (Figure 34).

Schulz and coworkers [119] reported the polymeric bithiophene-linked Co-salen catalyst 87 (Figure 35). The insoluble catalyst was employed in the dynamic HKR of epibromohydrin in THF. The catalyst was successfully reused in 11 subsequent catalytic runs, giving ring-opened products in good to excellent yield (60-99\%) and with consistent enantioselectivity (around $84 \% e e$ ). In a following study, the same group synthesized cyclic calixsalen catalyst $\mathbf{8 8}$, which contained a phenyl linkers instead of the previously used thiophene linkers (Figure 35) [120]. Catalyst 88 could be used either as a pure trimer, a pure tetramer or as a mixture. The best results were obtained with the tetramer, which afforded the ring-opened product in full conversion ( $>99 \%)$ and with high enantioselectivity $(92 \% e e)$ when used in the dynamic HKR of epibromohydrin in THF. The trimer exhibited significantly lower reactivities, both in terms of conversion and reaction rate. All the catalysts could be easily recovered from the reaction mixture by filtration and reused with maintained enantioselectivity, although the yield decreased after each run.

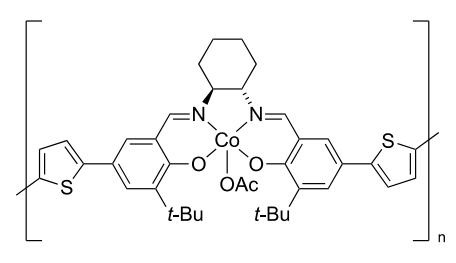

87

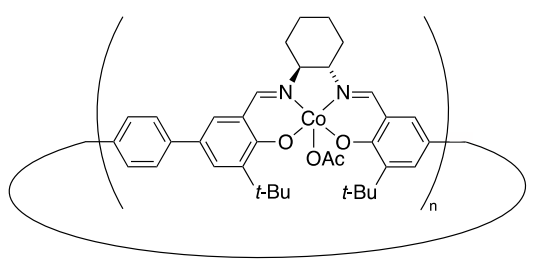

88: $n=3-4$

Figure 35. Polymeric Co-salen catalyst 87 and calixsalen Co catalyst 88.

In addition to dimers and macrocyclic oligomers, there are also some examples of other types of multi-salen structures. Jacobsen and coworkers synthesized dendrimeric Co-complexes based on polyamidoamine (PAMAM) central core containing 4, 8, and 16 salen moieties (represented by 8-dendrimer 89 in Figure 36), expecting that the dendritic framework would enforce the bimetallic cooperative pathway in the HKR of 1,2-epoxyhexane in THF [121]. The dendrimeric catalysts exhibited higher reactivities than monomeric and dimeric analogues 90 and 91 (40-42\% yield compared to $29-37 \%$ yield for the ring-opened product), as well as significantly higher rate constants. All catalysts (including 90 and 91) afforded the ring-opened product with excellent enantioselectivity $(>98 \% e e$ ). For the enantioenrichment of the unreacted epoxide, it was reported that catalyst 89 effected the HKR of 2-cyclohexyloxirane with $50 \%$ conversion and $98 \%$ ee.

Zheng and coworkers developed linear and cross-linked polymeric Co-salen complexes. They reported the linear polymeric Co-salen catalysts 92 and 93 (Figure 37), which catalyzed the HKR of propylene oxide, epichlorohydrin and phenyl glycidyl ether with excellent conversions (up to > $49 \%$ ) and $e e$ values (up to $98 \%$ for the unreacted epoxides and the 1,2-diols) in organic solvents (THF and $\mathrm{CH}_{2} \mathrm{Cl}_{2}$ ) or under solvent-free conditions [122]. No obvious differences in catalytic performances were observed between the two polymeric catalysts. 


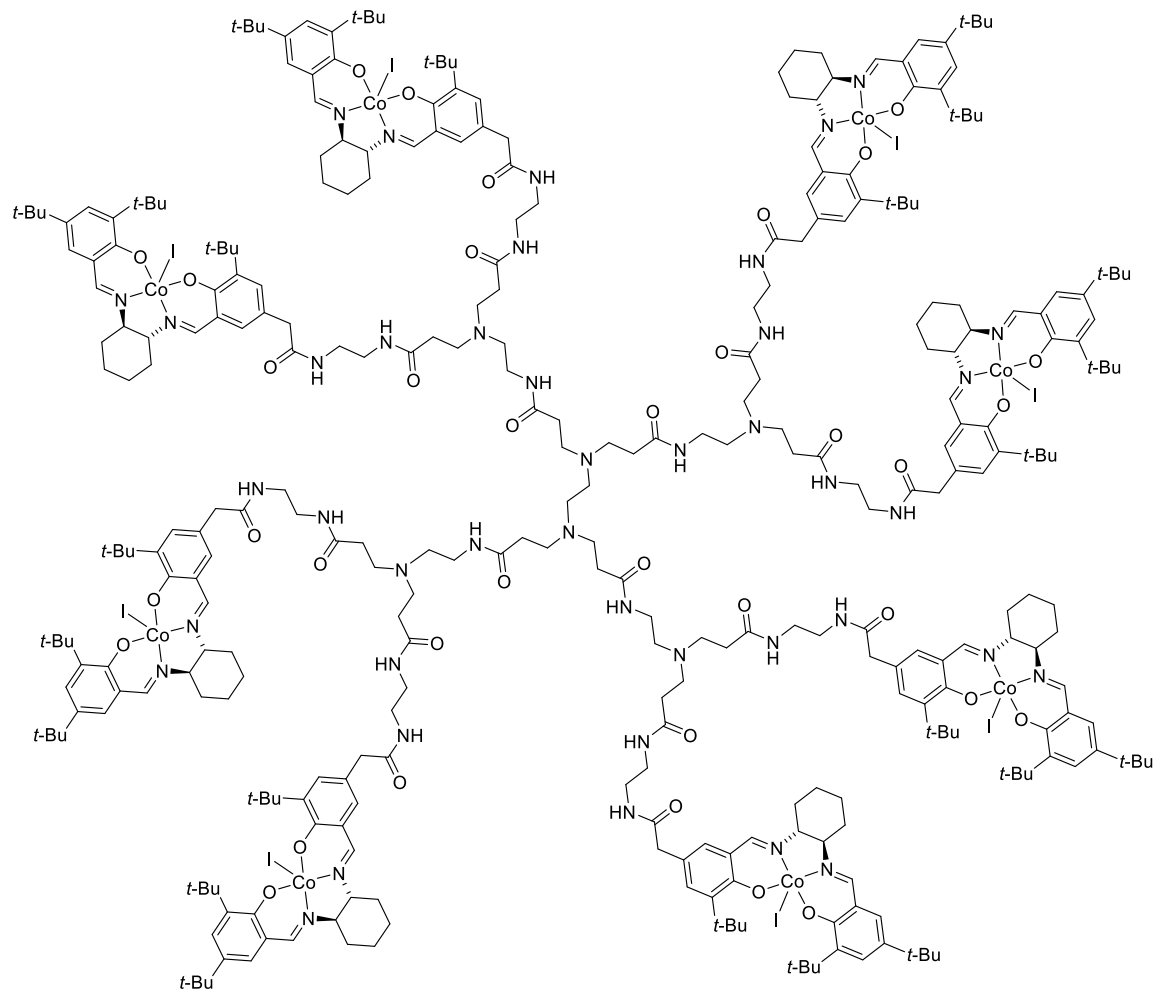

89
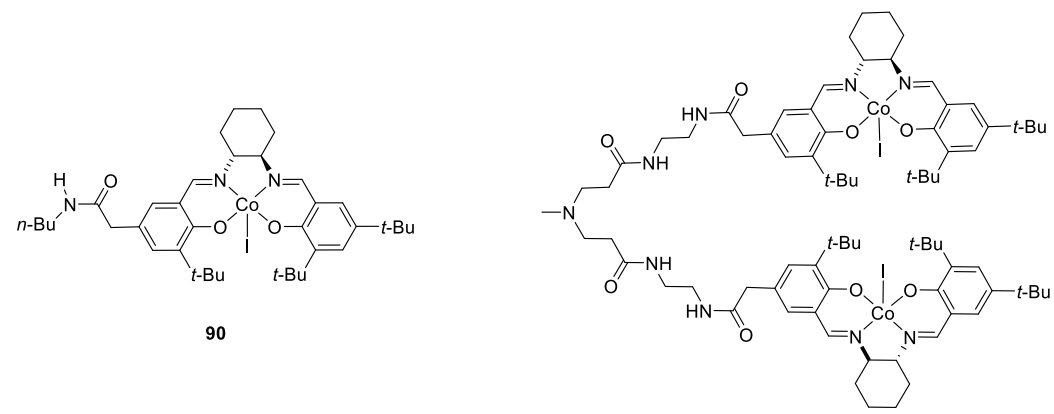

Figure 36. Polyamidoamine-based 8-dendrimer 89 and its monomeric (90) and dimeric (91) analogues.

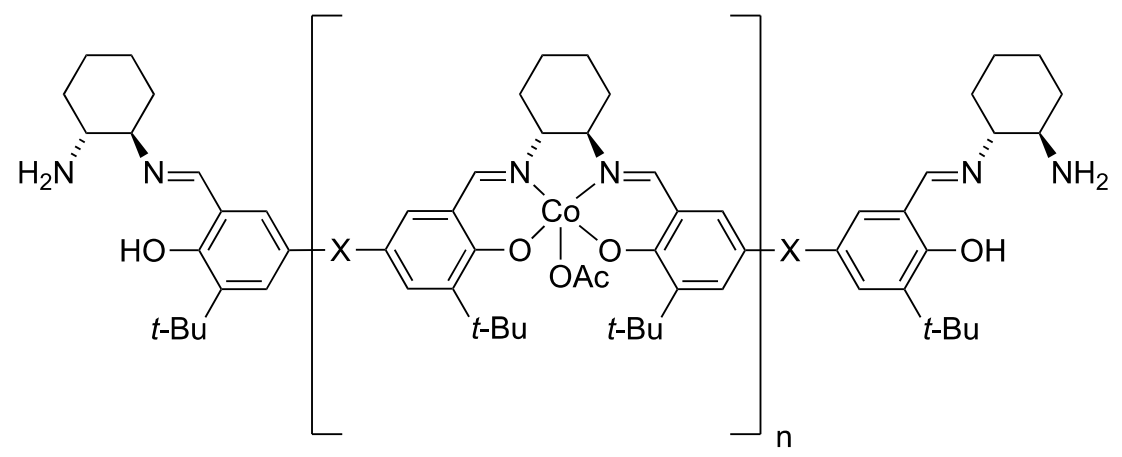

92: $\mathrm{X}=\mathrm{C}\left(\mathrm{CH}_{3}\right)_{2}$

93: $\mathrm{X}=\mathrm{CH}_{2}$

Figure 37. Linear polymeric Co-salen catalysts 92 and 93. 
The same group also reported the synthesis and catalytic performance of a set of cross-linked copolymers [123]. Catalyst 94 was synthesized by the condensation of enantiopure trans-1,2-diaminocyclohexane and a mixture of dialdehyde 95 and trialdehyde 96 (Scheme 43). A series of catalysts, oligomers 94 , were thus synthesized with different ratios of the two aldehydes (ranging from 100\% dialdehyde 95 to 100\% trialdehyde 96). The catalysts were applied in the HKR of epichlorohydrin, styrene oxide, and phenyl glycidyl ether under solvent-free conditions. Conversions of $43-53 \%$ were reported, with high enantioselectivities for both the ring-opened products and the unreacted epoxides (up to $>99 \%$ ee and up to $97 \%$ ee, respectively). The mixed cross-linked polymeric catalysts exhibited slightly higher reactivity than the catalysts based on either pure dialdehyde or pure trialdehyde. Recycling of the catalysts was unsuccessful due to the decomposition of the catalyst during the reaction. The authors attributed this to the hydrolysis of the ester groups under the reaction conditions.

$95+96 \underset{1)(R, R)-1,2 \text {-diamnocyclohexane, THF }}{\stackrel{\text { 2) } \mathrm{Co}(\mathrm{OAc})_{2} 4 \mathrm{H}_{2} \mathrm{O}, \mathrm{LPTS}, \text { toluene/methanol }}{\longrightarrow}}$ polymeric Co(III)salen catalyst 94

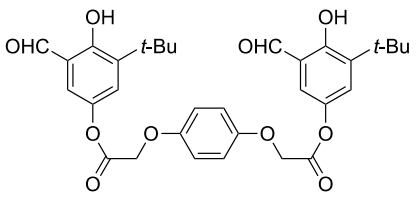

95

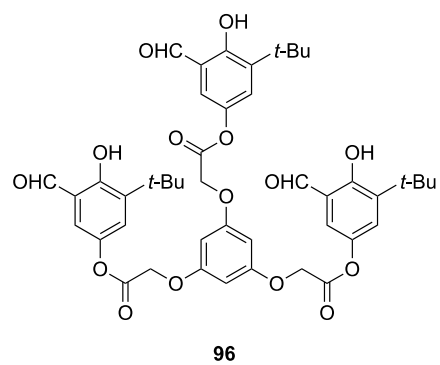

96

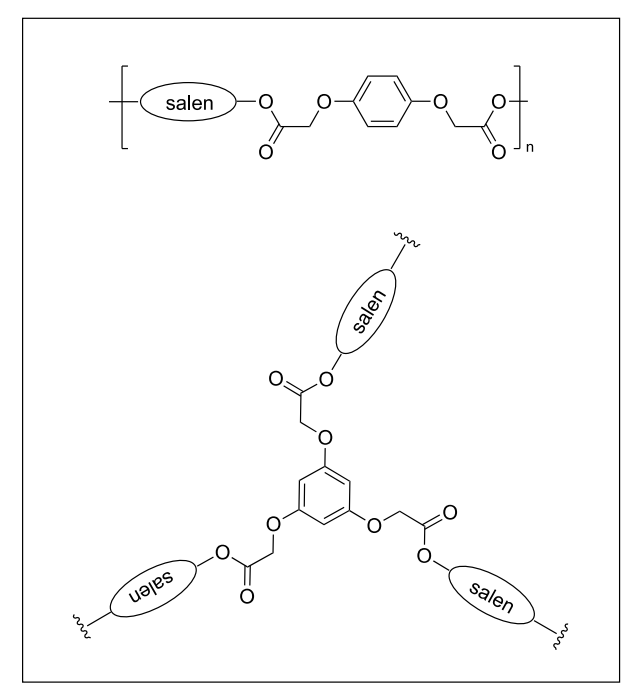

94

Scheme 43. The synthesis of cross-linked polymeric Co-salen catalyst 94 from dialdehyde 95 and trialdehyde 96. LPTS = lutidinium $p$-toluenesulfonate.

Weck and Jones [124] reported a set of homopolymerized and copolymerized Co(III)salen complexes, where the salen moieties were installed in a pendant-like fashion on the polystyrene chain (97-100 in Figure 38). Catalysts with different ratios of salen moieties and styrene moieties were investigated in the $\mathrm{HKR}$ of epichlorohydrin in $\mathrm{CH}_{2} \mathrm{Cl}_{2}$. All the catalysts gave $49-55 \%$ conversion and afforded the unreacted epoxide with excellent enantioselectivities ( $>99 \% e e)$. Copolymeric catalysts 99 and 100 exhibited improved reactivity compared to homopolymeric catalyst 97 ( $1 \mathrm{~h}$ reaction time compared to $2 \mathrm{~h}$ ). The authors hypothesized that the lower ratio of the salen moieties on the polystyrene chain could make the catalytic sites more accessible to the substrate. The same group also evaluated the optimal flexibility of polymeric Co-salen catalysts by synthesizing a series of homopolymeric catalysts with different lengths of the linker connecting the Co-salen complexes to the polymeric backbone (catalyst 101-104 in Figure 38) [125]. The catalysts were employed in the HKR of epichlorohydrin. Kinetic studies revealed that a 6-atom distance (corresponding to $m=1$, catalyst 102) between the polymer backbone and salen unit gave the highest reactivity and enantioselectivity. Catalyst $\mathbf{1 0 2}$ executed the successful HKR of a number of terminal epoxides under solvent-free conditions and with low catalyst loading (0.01-0.1 mol\%), affording unreacted epoxides in $43-46 \%$ yield and $\geq 98 \%$ $e e$. These papers illustrate the importance of the flexibility and composition of polymer-supported Co-salen catalysts for the HKR of terminal epoxides. 


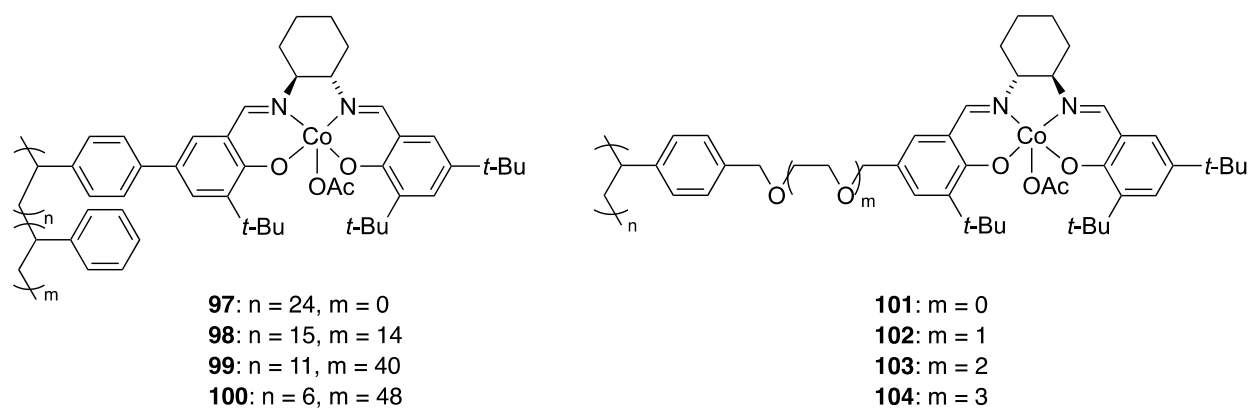

Figure 38. Homo- and copolymerized Co-salen complexes $\mathbf{9 7 - 1 0 0}$ and homopolymerized Co-salen complexes 101-104.

Weberskirch and coworkers [126] synthesized the first water-soluble amphiphilic block copolymeric Co-salen catalyst for the employment in HKR reactions (Figure 39) epoxides. The polymeric complex $\mathbf{1 0 5}$ could aggregate into micellar assemblies in aqueous environment, forming a hydrophobic core with a high local concentration of the active catalyst. The so-formed catalyst $\mathbf{1 0 5}$ enabled the efficient and enantioselective HKR of a number of terminal aryl epoxides and glycidyl ethers. The reactions proceeded with $50-54 \%$ conversion and with high-to-excellent enantioselectivities (up to $95 \%$ ee for the 1,2-diols and $>98 \%$ ee for the unreacted epoxides). The catalyst could be recycled and reused in four successive cycles with consistent $e e$ values for the unreacted epoxides, although the reaction time needed to be increased after each cycle to maintain the yield.

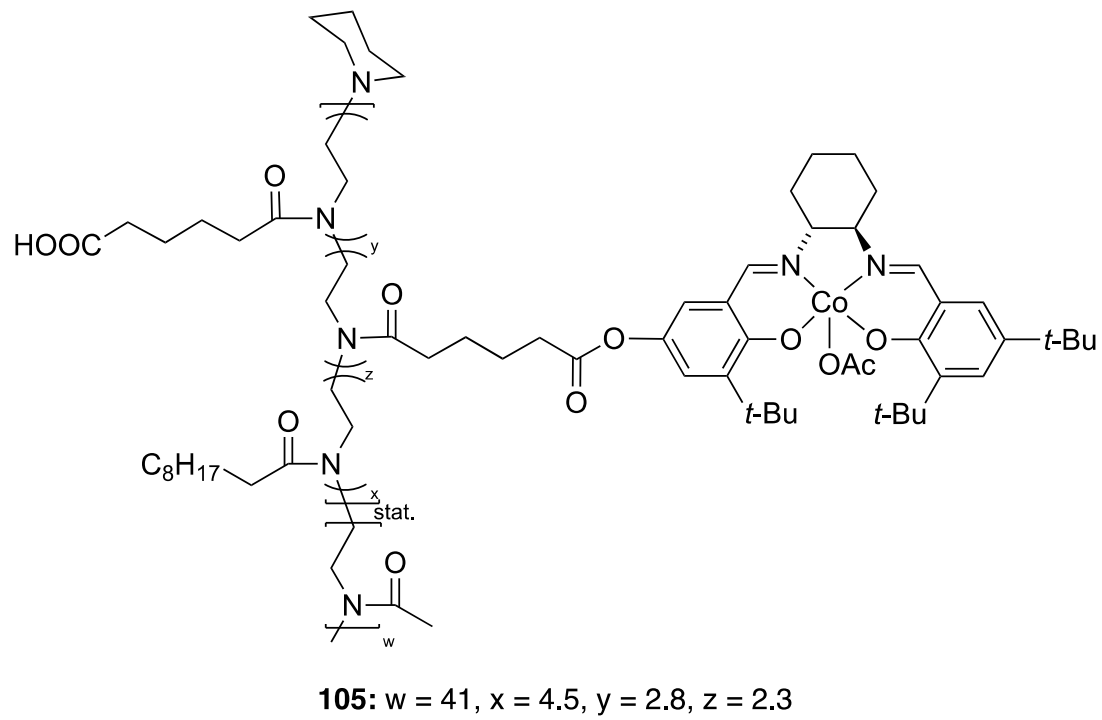

Figure 39. Amphiphilic copolymerized Co-salen catalyst 105.

As described above, covalently linking metal-salen moieties together is one approach to obtaining multi-metallic catalysts. However, these larger motifs often require considerable synthetic efforts. In addition, many of the oligomeric complexes and polymers are prepared and used as mixtures in the catalysis, making it more complicated to elucidate the most active structure and understand how efficient catalysis is achieved. One alternative that could reduce the synthetic cost is the use of multi-metallic metal-salen assemblies based on non-covalent interactions. The most attractive case is one where the assembly can be completely controlled and only one well-defined catalyst is formed.

Following this line, Kim and coworkers [127] developed a series of dimeric catalysts where two identical Co-salen complexes were connected through the coordination of one oxygen of each salen unit to an $\mathrm{Al}(\mathrm{III})$-containing Lewis acid (catalyst 54 in Scheme 34). The dimeric catalysts could induce high reactivities (41-46\% yield for the ring-opened products and $40-46 \%$ yield for the unreacted epoxides) 
and enantioselectivities (up to $86 \%$ ee for the ring-opened products and up to $99 \%$ ee for the unreacted epoxides) in the HKR of epichlorohydrin, 1,2-epoxybutane, and glycidol, whereas monomeric analogues catalyzed the reactions with significantly lower reactivities and enantioselectivities. The catalysis could be performed either in THF or under solvent-free conditions. The same strategy was also extended to Lewis acids of other group 13 elements (catalyst 55 and $\mathbf{5 6}$ in Scheme 34) [128]. The dimeric Co-salen complex 55 (linked by $\mathrm{GaCl}_{3}$ ) was employed in the HKR of a wide range of 3-substituted propylene oxides and glycidyl ethers, affording unreacted epoxides in 40-49\% yield and $97-99.8 \%$ ee. Ring-opened products were obtained in $42-50 \%$ yield and $>85 \%$ ee. The reactions were performed under solvent-free conditions in THF or in a $\mathrm{CH}_{2} \mathrm{Cl}_{2}$ /THF mixture. Kinetic studies showed the participation of both intramolecular and intermolecular pathways in the HKR reactions and significantly higher reaction rates for dimeric complexes than monomeric analogues. Other Lewis acids $\left(\mathrm{ZnCl}_{2}, \mathrm{FeCl}_{3}, \mathrm{SnCl}_{4}\right.$, etc.) have also been attached to $\mathrm{Co}$ (III)salen complexes, and the so-formed monomeric catalysts exhibited improved reactivities in comparison with monosalen complex 64 (Figure 26) in the HKR of terminal epoxides [129-131].

Supramolecular interactions, such as aromatic donor-acceptor interactions and hydrogen bonding, have also been utilized to construct multi-metallic catalysts with controlled structures. The previously mentioned aromatic donor-acceptor complexes 42-44 (Figure 19) also worked very well as catalysts for the HKR of various terminal epoxides such as styrene oxide and sterically hindered tert-butyloxirane in $\mathrm{CHCl}_{3}$ or $\mathrm{CCl}_{4}[70]$. The resolved epoxides were isolated in high yields $(>40 \%)$ and enantioselectivities (up to $99 \%$ ee).

Hong and coworkers [132] designed and synthesized bis-urea-functionalized Co-salen complexes capable of forming self-assembled hydrogen-bonding structures in solution. The complex with $p-\mathrm{CF}_{3}$-phenyl substituents on the urea groups gave the highest rate constant in the HKR of epichlorohydrin (catalyst 106 in Figure 40). Catalyst 106 was investigated in the HKR of epichlorohydrin, allyl glycidyl ether, 1,2-epoxybutane and 1,2-epoxyhexane under solvent-free conditions, affording unreacted epoxides in $41-43 \%$ yield and 99\% ee. In addition, complex 106 showed significantly higher reactivity than the monosalen complex 64 (Figure 26) at the same catalyst loading. Kinetic and self-association studies supported the hypothesis that the observed rate enhancement could be attributed to the enforcement of the bimetallic mechanism by the proximal self-association of Co-salen units through urea-urea hydrogen-bonding.

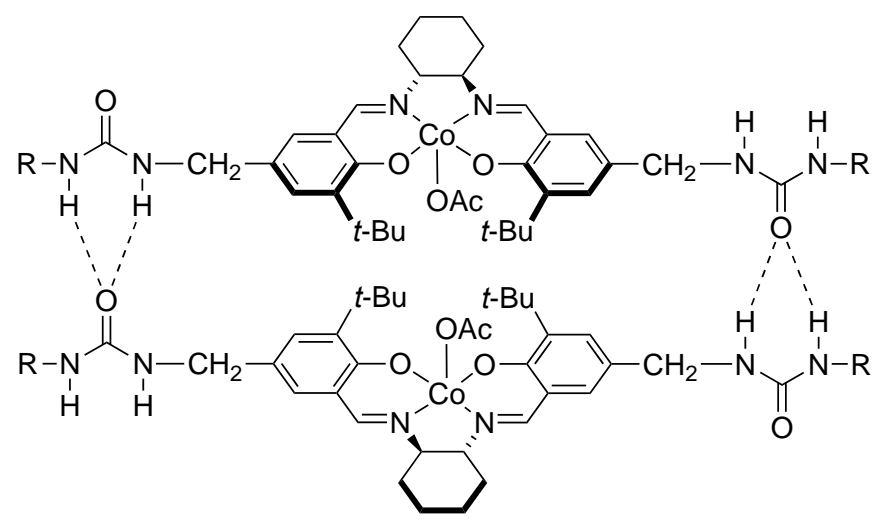

Figure 40. Bis-urea-functionalized Co-salen catalyst 106, displayed as hydrogen-bonded dimer.

Another approach to achieving more efficient catalysis is to utilize space constraints. Li and Yang [133] placed homogenous monosalen catalyst 64 (Figure 26) in a nanocage of mesoporous silica SBA-16 (one catalyst per cage) via the ship in a bottle strategy. A key step was tuning the size of the pores so that the reactants used for synthesizing the catalytically active complex (salen ligand and cobalt salt) were allowed to enter the nanocage but the formed complex was hampered from escaping. The so-immobilized catalyst was easy to recycle and was effective for up to 11 successive 
runs, but the system gave lower enantioselectivity and reactivity than reactions performed with catalyst 64 under homogeneous conditions. To enable the bimetallic cooperative pathway, the same group described a new catalytic system where two $\mathrm{Co}$-salen complexes were accommodated in one nanocage (Scheme 44) [134]. With nanoreactor 107, the HKR of propylene oxide in $\mathrm{CH}_{2} \mathrm{Cl}_{2}$ could be completed with full conversion (50\%) and excellent enantioselectivity ( $98 \%$ ee for both the unreacted epoxide and the 1,2-diol) at very low catalyst loading (1:12000 Co-salen/epoxide).

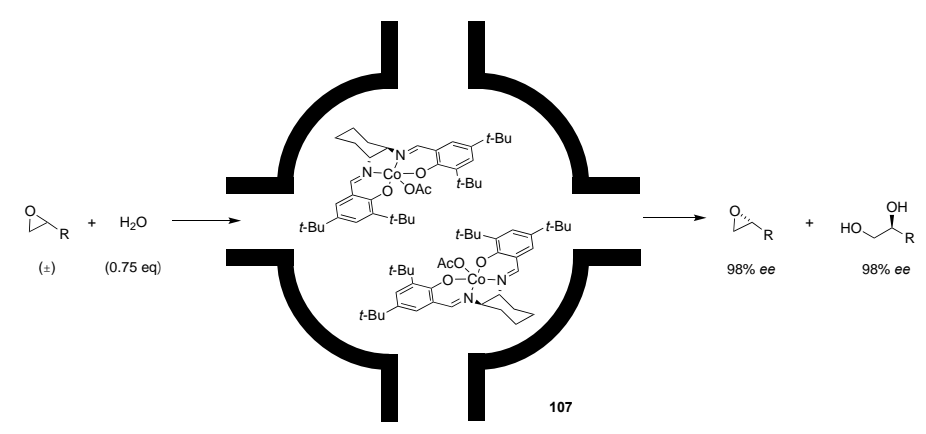

Scheme 44. The hydrolytic kinetic resolution of terminal epoxides performed in nanoreactor $\mathbf{1 0 7}$ in $\mathrm{CH}_{2} \mathrm{Cl}_{2}$.

\subsubsection{Immobilized Catalysts}

In the HKR of terminal epoxides catalyzed by $\mathrm{Co}(\mathrm{III})$ salen complexes, the main recycling procedure for most homogenous catalysts is to distill off all the volatile fractions and collect the solid residue. However, distillation is not an ideal separation method for industrial purposes due to the large amount of energy needed and may be limited by the stability of the catalysts at elevated temperatures. In light of this, much research has been aimed at improving the separation of the catalyst and products.

One strategy is to use a fluorous biphasic catalytic system, where employing fluorinated catalysts allows the reaction to be worked up through an organic/fluorocarbon phase separation. The products dissolve into the organic phase and the F-containing catalyst dissolves into the fluorocarbon phase. This concept was explored by Pozzi and coworkers [135,136], who synthesized a number of fluorinated Co-salen complexes for the HKR of terminal epoxides. Some of the fluorinated complexes worked well, but some complexes suffered from a compromise between the solubility in organic solvent to induce the reaction and the solubility in fluorocarbon to achieve the biphasic extraction. Other approaches to achieving improved catalyst separation include the use of ionic liquids and solvent-resistant nanofiltration, both of which have been applied successfully $[137,138]$.

Still, the most investigated approach is the immobilization of the catalyst on polymers and inorganic materials. Following this line, Jacobsen and coworkers [139] investigated Co-salen complexes immobilized on polystyrene resin. The hydroxymethylpolystyrene-supported heterogeneous catalyst 108 (Figure 41) was applied in the HKR of epichlorohydrin and 4-hydroxy-1-butene oxide, two substrates that were not suitable for distillation. The HKR of epichlorohydrin was conducted in $\mathrm{CH}_{2} \mathrm{Cl}_{2}$, and the HKR of 4-hydroxy-1-butene oxide was run in THF. Both the ring-opened products and the unreacted epoxides were obtained in good yields ( $36-52 \%$ and $38-41 \%$, respectively) and with high enantioselectivities (up to $>99 \%$ ee for the unreacted epoxides and up to $95 \%$ ee for the ring-opened products). Catalyst 108 was also used for the dynamic HKR of epibromohydrin, affording the ring-opened product in $94 \%$ yield and $96 \%$ ee. The catalyst could be recovered by simple filtration and recycled up to five times with no apparent loss of reactivity or enantioselectivity.

Kirschning and coworkers [140] reported on a related system, where the Co-salen complex was immobilized on a chloromethylpolystyrene resin (catalyst 109 in Figure 41). Catalyst 109 was investigated in the HKR of epichlorohydrin, styrene oxide, and phenyl glycidyl ether in THF, affording ring-opened products and unreacted epoxides in similar or slightly inferior yields and enantioselectivities compared those obtained with homogeneous Co-salen catalyst 64 (Figure 26). 
Catalyst 109 was also effective in catalyzing the dynamic HKR of epibromohydrin in THF, giving the corresponding diol with $94 \%$ yield and $95 \% e e$. The same study also included the immobilization of Co-salen complexes on a porous glass/polymer composite material inside a "PASSflow" reactor [141] which was used to study the catalysis of the dynamic HKR of epibromohydrin in THF under continuous-flow conditions. With reactivation after each run, the modified microreactor could be used for four consecutive runs, affording the ring-opened product in 76-87\% yield and 91-93\% ee [140].

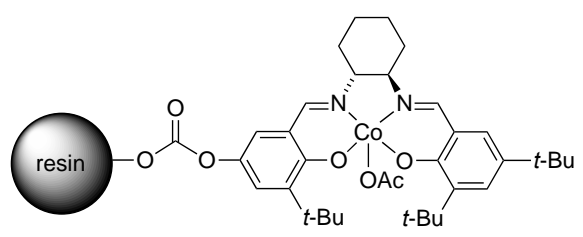

108

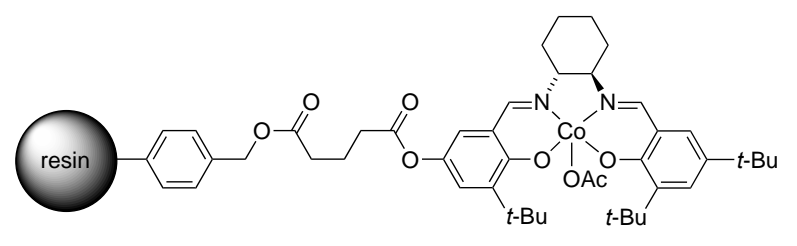

109

Figure 41. Resin-supported Co-salen catalysts 108 and 109.

Weck and coworkers [142] synthesized polystyrene resin-supported dendronized Co-salen catalyst 110 (Figure 42) which was employed in the HKR of epichlorohydrin, 1,2-epoxyhexane, allyl glycidyl ether, and styrene oxide. Reactions performed with very low catalyst loading $(0.04-0.06 \mathrm{~mol} \% \mathrm{Co})$ and under solvent-free conditions afforded unreacted epoxides in high yields (40-47\%) and with excellent enantioselectivities ( $>99 \%$ ee). The excellent catalytic properties were attributed to the flexible linkers and the dendronized framework which probably assisted to promote the cooperative interactions among Co-salen sites and facilitate the bimolecular pathway. Catalyst $\mathbf{1 1 0}$ could be used in five successive HKR reactions with maintained reactivity and enantioselectivity.

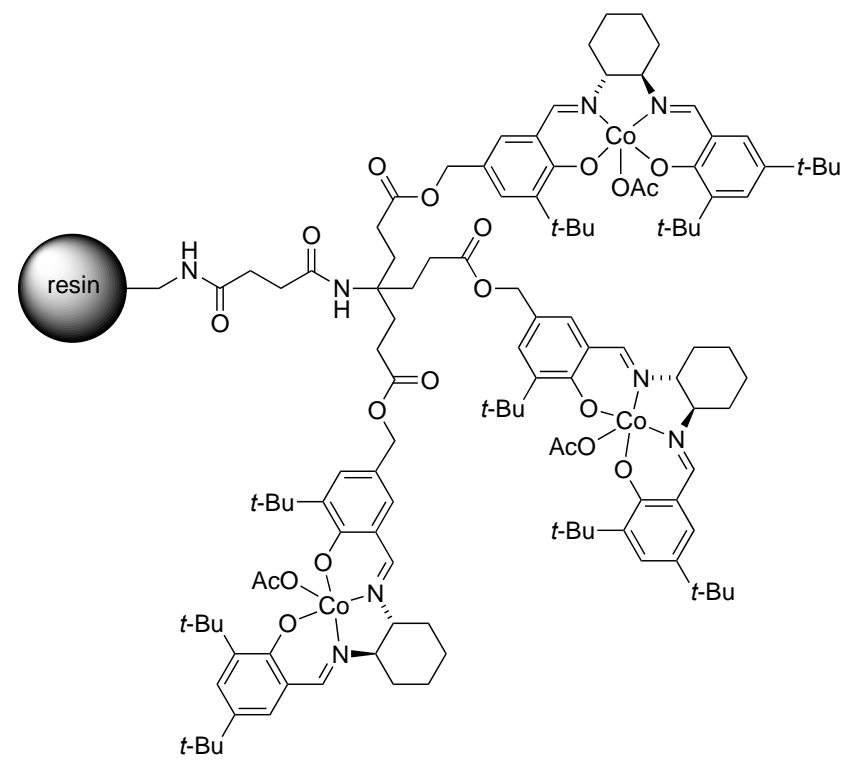

110

Figure 42. Resin-supported dendronized Co-salen catalyst 110. 
Weck and coworkers [143] grafted their macrocyclic oligomeric catalyst 76 (Figure 31) on a polystyrene resin. The resulting catalyst 111 (Figure 43) was used in the solvent-free HKR of epichlorohydrin and 1,2-epoxyhexane, achieving complete resolution ( $\geq 50 \%$ conversions and $99 \%$ ee of the unreacted epoxides) in $3 \mathrm{~h}$ or less with only $0.01 \mathrm{~mol} \%$ Co loading. As such, catalyst 111 is the most efficient heterogeneous catalyst for the HKR of terminal epoxides to date. The catalyst could be recovered by filtration and reused up to five times with maintained enantioselectivity, although the reaction time required to reach full conversion increased after each run. A noteworthy observation was that the use of an excess of water (six equivalents instead of 0.6) led to a significant rate enhancement. The enhanced reactivity was attributed to the formation of a biphasic reaction system, in which the formed diol partitions into the aqueous phase, leaving a higher concentration of epoxide and catalyst in the organic phase.

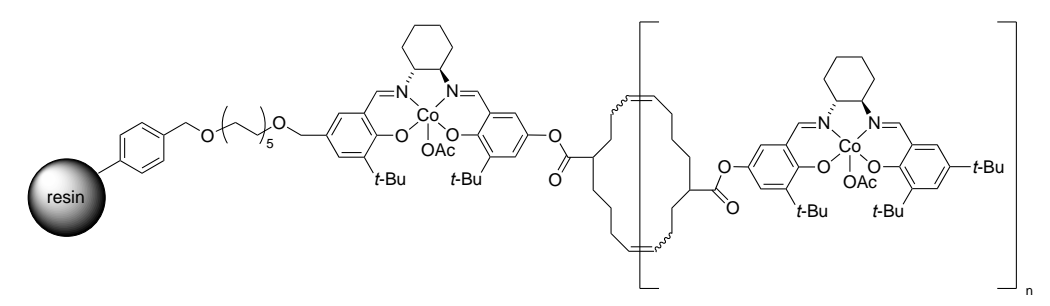

111

Figure 43. Resin-supported macrocyclic oligosalen catalyst 111 based on oligomer $\mathbf{7 6}$ (Figure 31).

In addition to the immobilization on polystyrene resin, Co-salen catalysts have also been immobilized on different silica-based supports. Compared to polystyrene, silica is a more rigid support and thus potentially better suited for use as a stationary phase in continuous-flow apparatus. Jacobsen and coworkers covalently immobilized a Co-salen complex on silica (Figure 44) [139]. The resulting catalyst $\mathbf{1 1 2}$ was employed as stationary phase in a continuous-flow system for the HKR of 4-hydroxy-1-butene oxide. Employing $\mathrm{THF} / \mathrm{H}_{2} \mathrm{O}$ as mobile phase, the reaction afforded the corresponding triol in a good yield (37\%) and with high enantioselectivity $(94 \%$ ee).

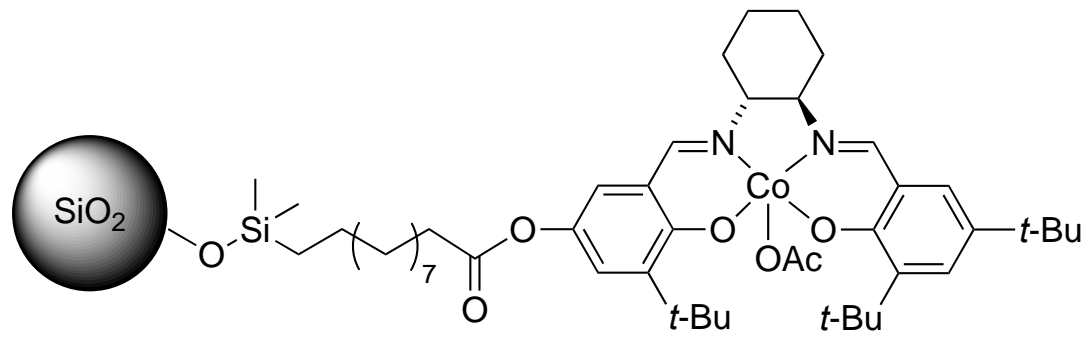

112

Figure 44. Silica-supported catalyst 112.

Jones and coworkers [144] synthesized a series of heterogeneous polymeric catalysts by immobilizing Co-salen complexes on CAB-O-SIL silica-supported polymer brushes (Figure 45). The HKR of epichlorohydrin in $\mathrm{CH}_{2} \mathrm{Cl}_{2}$ was employed as a model reaction to compare the catalytic properties of polymeric catalyst $\mathbf{1 1 4}$ with the less flexible polymeric analogue 113, the homogenous catalyst 64 (Figure 26), and the monosalen analogue 115. Amongst all the catalysts, polymer 114 exhibited the highest reactivity and enantioselectivity (close to $50 \%$ conversion and $>99 \%$ ee for both the ring-opened product and the unreacted epoxide). The catalyst with a less flexible linker, polymer 113, was less reactive than 114, but still exhibited high enantioselectivity $(>99 \%$ ee for the ring-opened product and $95 \%$ ee for the unreacted epoxide). Monomeric catalyst 115 performed worst, both in terms of reactivity and enantioselectivity, which was attributed to its low local concentration of Co-salen sites 
and thus diminished Co-salen cooperativity. Catalyst 114 was also investigated in recycling studies, where the enantioselectivity could be maintained over five catalytic runs, although the reaction rate decreased after each subsequent run. The FTIR and elemental analysis studies indicated that the loss of reactivity might be caused by cleavage of the salen ligand under the reaction condition.
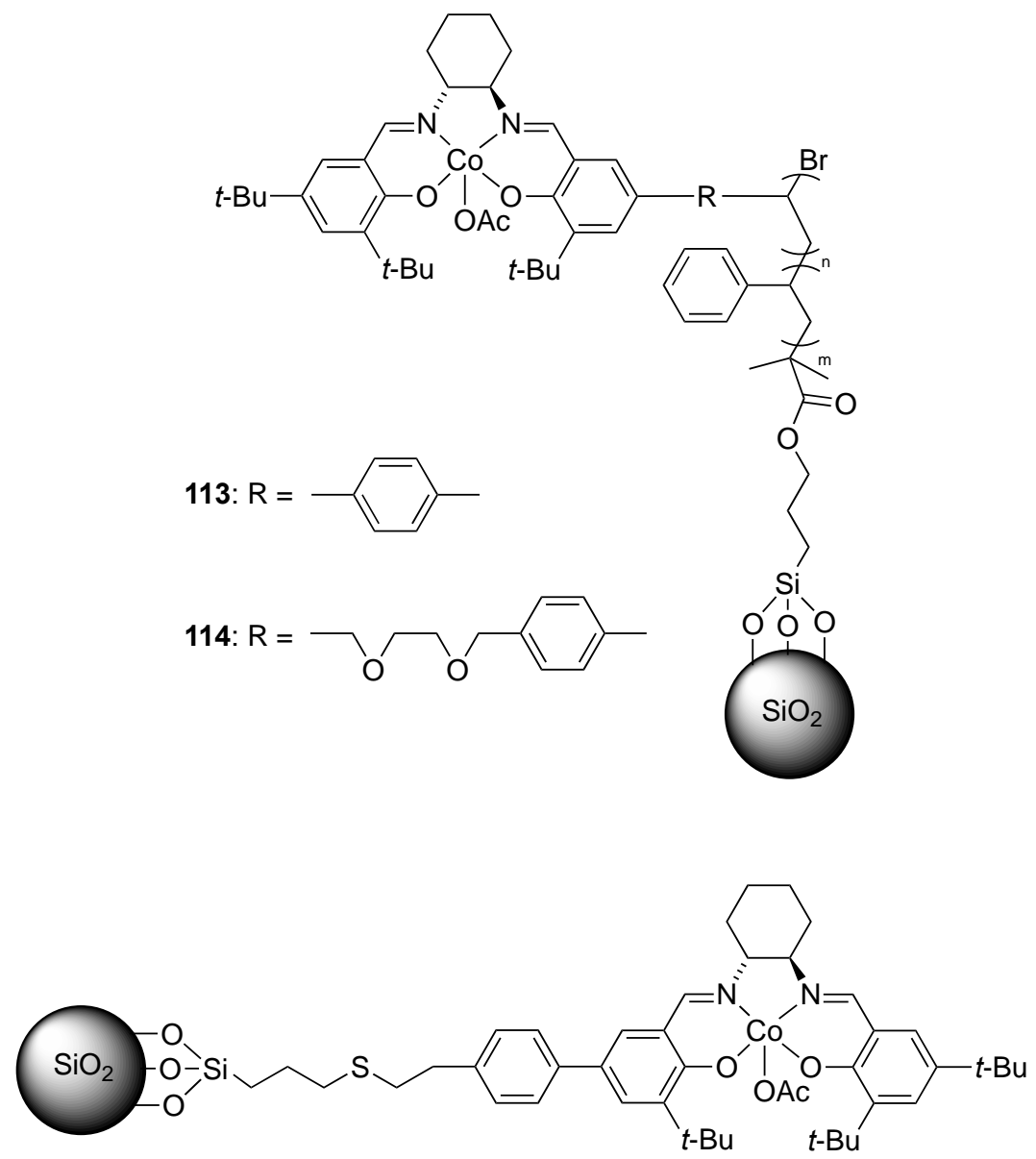

115

Figure 45. Silica-immobilized copolymeric Co-salen complexes (113 and 114) and silica-immobilized monomeric complex (115).

Jacobsen's group [145] successfully immobilized Co(III)salen complexes on gold colloids through an exchange reaction between thiol-containing $\mathrm{Co}$-salen complexes and $n$-octanethiolates, the latter pre-coordinated to the gold colloid (Figure 46$)$. With low catalyst loading $(0.01 \mathrm{~mol} \% \mathrm{Co})$, the so-immobilized catalyst $\mathbf{1 1 6}$ catalyzed the solvent-free HKR of 1,2-epoxyhexane ten times faster than the non-immobilized analogue 117 (Figure 46), achieving complete resolution within $5 \mathrm{~h} \mathrm{( \geq 50 \%}$ conversion and $>99 \%$ ee of the unreacted epoxide). The catalyst could be recovered by filtration through centrifugal filter units and recycled several times with maintained reactivity and enantioselectivity, only requiring re-oxidation after six consecutive reaction cycles.

Kim and coworkers $[65,128]$ published several studies on the immobilization of salen catalysts on inorganic materials such as mesoporous silicate or alumosilicate. A series of monomeric and dimeric Co-salen catalysts were grafted onto mesoporous silica MCM-41 (Figure 47). In the solvent-free HKR of epichlorohydrin, styrene oxide, and 1,2-epoxyhexane, each of the silica-supported monosalen catalysts 118-121 exhibited decreased reactivities but comparable enantioselectivities compared to homogenous monosalen catalyst 63 (Scheme 40) [65]. In a later study, Kim and coworkers [128] immobilized 
dimeric Co-salen complexes 54-56 (Scheme 34) on MCM-41 (catalysts 122-124 in Figure 47). Similar to what was observed for the immobilized monosalen catalysts 118-121, the immobilized dimeric catalysts 122-124 also exhibited decreased reactivities but comparable enantioselectivities in the HKR of epichlorohydrin compared to homogeneous analogues $\mathbf{5 4 - 5 6}$ (Scheme 34). In both these cases, it is clear that the immobilization hindered efficient catalysis. One explanation could be the relatively short linkers used, as previous studies clearly show that the flexibility and length of the linker is of paramount importance in enabling efficient catalysis. Another possible explanation could be the choice of mesoporous silica as solid support.

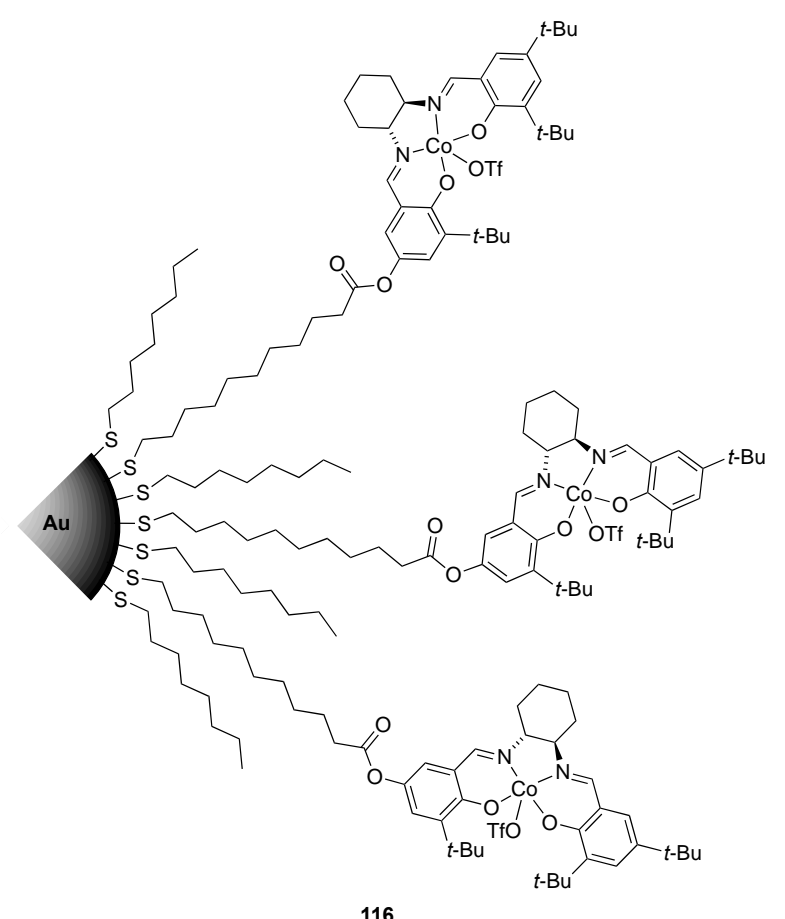

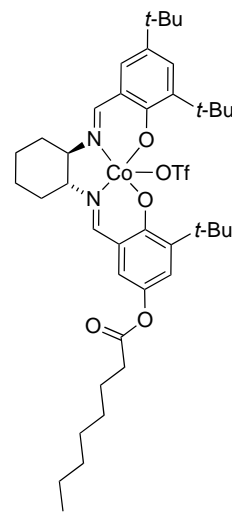

117

Figure 46. Au colloid-supported Co-salen catalyst 116 and non-immobilized analogue 117.

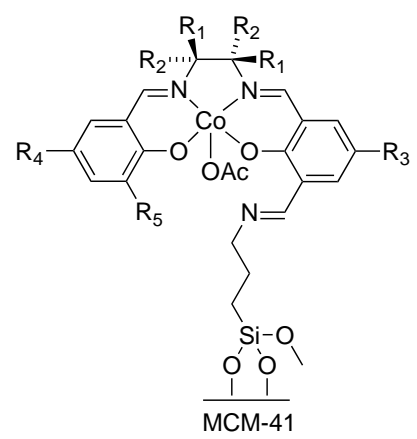

118: $R_{1}=P h, R_{2}=R_{3}=H, R_{4}=R_{5}=t-B u$

119: $\mathrm{R}_{1}=-\left(\mathrm{CH}_{2}\right)_{4}-, \mathrm{R}_{2}=\mathrm{R}_{3}=\mathrm{H}, \mathrm{R}_{4}=\mathrm{R}_{5}=t-\mathrm{Bu}$

120: $\mathrm{R}_{1}=-\left(\mathrm{CH}_{2}\right)_{4^{-}}, \mathrm{R}_{2}=\mathrm{H}, \mathrm{R}_{3}=t-\mathrm{Bu}, \mathrm{R}_{4}=\mathrm{R}_{5}=\mathrm{H}$

121: $R_{1}=-\left(\mathrm{CH}_{2}\right)_{4}^{-}, \mathrm{R}_{2}=\mathrm{R}_{3}=H, \mathrm{R}_{4}=\mathrm{R}_{5}=t-\mathrm{Bu}$

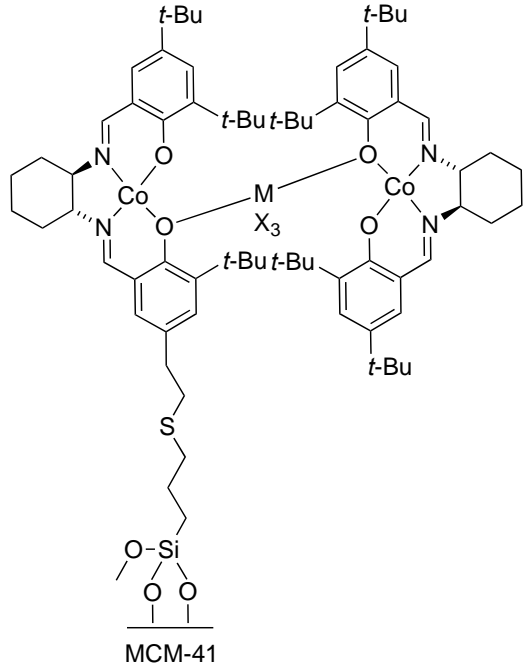

122: $\mathrm{M}=\mathrm{Al}, \mathrm{X}=\mathrm{Cl}$

123: $\mathrm{M}=\mathrm{Ga}, \mathrm{X}=\mathrm{Cl}$

124: $M=\ln , X=C l$

Figure 47. MCM-41-supported salen catalysts 118-124. 
To this end, Kim and coworkers [146] studied the effect of the pore size and structure of the silica support on the catalytic activity. Dimethylcarbonate (DMC) was used to partially desilylate mesoporous silica, and the resulting material was then used as a solid support for polymeric Co-salen complexes. The so-formed catalysts exhibited increased reactivities and enantioselectivities in the HKR of terminal epoxides compared to catalysts prepared without pretreatment with DMC.

Kim and coworkers [147] also investigated other mesoporous materials such as SBA-15 and SBA-16 with a wider range of pore sizes and higher stability. Co-salen complexes were attached on sulfonate functionalized SBA-16 through electrostatic interaction between the cobalt and the sulfonate (Figure 48). The so-formed catalyst $\mathbf{1 2 5}$ was employed in the HKR of epichlorohydrin in THF, affording the unreacted epoxide in $44 \%$ yield and $98 \%$ ee, although similar results were also obtained with homogeneous analogues.

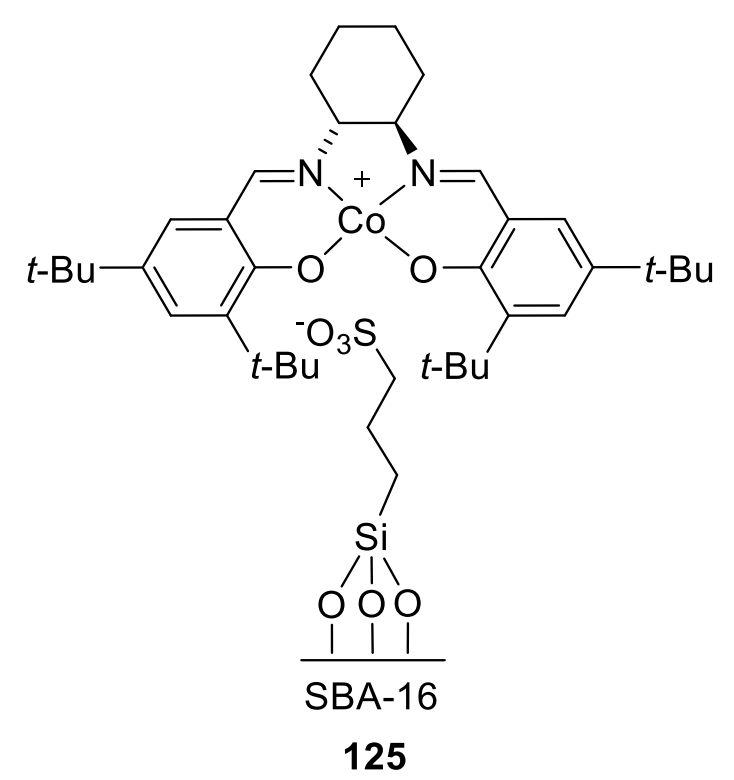

Figure 48. SBA-16-supported Co-salen catalyst 125.

In a later study, the same group reported another type of non-covalent immobilization based on interactions between fluorine-bearing catalysts and different acidic sites in mesoporous Al-SBA-15 (Figure 49) [148,149]. The so-formed catalyst 126 was investigated in the HKR of terminal epoxides in THF, affording unreacted epoxides in full conversions $(50 \%)$ and with good-to-excellent enantioselectivities (up to $99 \%$ ee). However, homogeneous analogues gave comparable results in shorter reaction times.

(a)

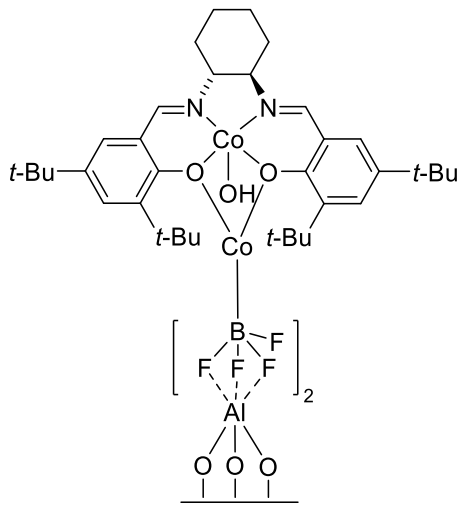

(b)

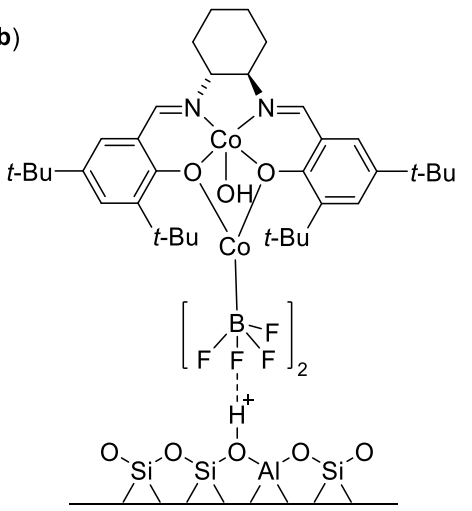

Figure 49. Al-SBA-15-supported Co-salen catalyst 126, formed by interactions with (a) Lewis acidic sites and (b) Brønsted acidic sites. 


\subsubsection{Mechanistic Studies}

Several practical and theoretical studies have been carried out to investigate the mechanism of the HKR of terminal epoxides by Co(III)salen complexes. Even though the cooperative mechanism has been supported by many studies [28,29], a few features about this reaction required further investigation. One of them was the influence of different counterions on the reactivity of the catalyst.

In most of the early papers regarding the HKR of epoxides using $\mathrm{Co}(\mathrm{III})$ salen catalysts, the acetate anion was employed as the counterion. However, later studies showed that the reaction could be promoted or demoted by changing the counterion [110,150]. Jacobsen and coworkers [151] performed extensive mechanistic studies in an effort to understand and explain this effect, and to identify more active catalysts for the HKR of terminal epoxides. As illustrated in Scheme 45, two possible reaction pathways that compete with each other are proposed. When the counterion $(X)$ is completely non-nucleophilic (e.g., hexafluoroantimonate), the reaction undergoes a less selective monometallic pathway, where the epoxide is activated by the $\mathrm{Co}(\mathrm{III}) \operatorname{salen}(\mathrm{X})$ and attacked by water (Scheme 45 , top). When the counterion is nucleophilic (e.g., chloride or acetate), a bimetallic cooperative pathway takes place (Scheme 45, bottom). In the latter pathway, the active nucleophile Co(III)salen $(\mathrm{OH})$ is formed irreversibly from an initial hydrolysis of $\mathrm{Co}(\mathrm{III}) \operatorname{salen}(\mathrm{X})$. The bimetallic pathway being the major pathway is supported by kinetic studies by Kleij and Jacobsen [114,139] and DFT calculations by $\mathrm{Li}$ [152]. In the bimetallic pathway, the key to obtaining high rates in the HKR reaction is to have an equal ratio of $\mathrm{Co}(\mathrm{III})$ salen $(\mathrm{X})$ (which could activate the epoxide) and $\mathrm{Co}(\mathrm{III})$ salen $(\mathrm{OH})$ (which could act as nucleophile). Kinetic studies of a number $\mathrm{Co}$-salen complexes with different counterions showed that $\mathrm{Co}(\mathrm{III})$ salen(OTs) achieved full conversion fastest in the HKR of 1,2-epoxyhexane. This was attributed to its slower coordination to the epoxide, thus maintaining a more favorable $\mathrm{Co}-\mathrm{X} / \mathrm{Co}-\mathrm{OH}$ ratio throughout the reaction [151]. The experimentally observed trends of reaction rates between different counterions $(\mathrm{OTs}>\mathrm{OAc}>\mathrm{Cl}$ ) were also supported by DFT calculations performed by Sherrill and coworkers [153].

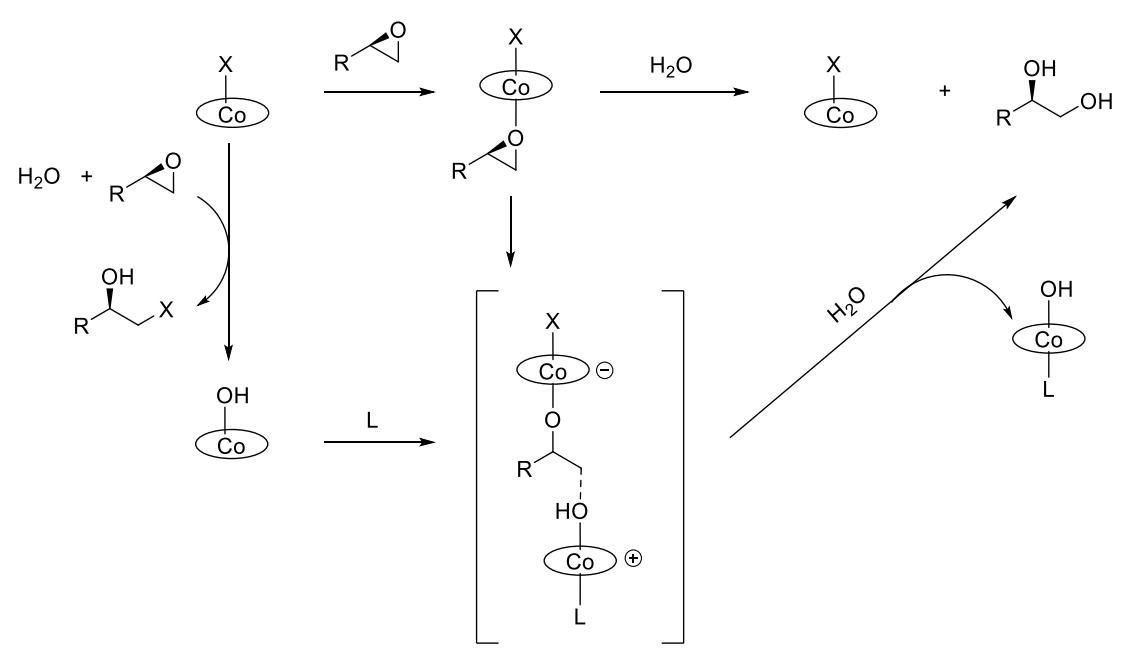

Scheme 45. Proposed reaction pathways for the HKR of terminal epoxides catalyzed by Co(III)salen complexes [151]. (top) A monometallic pathway with one Co-salen catalyst activating the epoxide and $\mathrm{H}_{2} \mathrm{O}$ acting as a nucleophile. (bottom) A bimetallic cooperative pathway with initial formation of $\mathrm{Co}(\mathrm{III})$ salen $(\mathrm{OH})$ from the reaction between $\mathrm{Co}(\mathrm{III}) \operatorname{salen}(\mathrm{X}), \mathrm{H}_{2} \mathrm{O}$, and epoxide, followed by a reaction between the so-formed activated nucleophile $\mathrm{Co}(\mathrm{III}) \operatorname{salen}(\mathrm{OH})$ and an epoxide activated by another $\mathrm{Co}(\mathrm{III}) \operatorname{salen}(\mathrm{X})$ catalyst.

Another mechanistic mystery concerned the deactivation of the catalyst. In the original paper where Co-salen 63 (Scheme 40) was first employed in the HKR of epoxides, the catalyst needed to be regenerated by treatment with acetic acid in air before it could be reused [109]. One of the possible 
explanations was that $\mathrm{Co}(\mathrm{III})$ was reduced to $\mathrm{Co}(\mathrm{II})$ through a one-electron transfer oxidation during the reaction. Although many of the later $\mathrm{Co}-$ salen catalysts did not require reactivation, the reason for the occasionally observed deactivation remained unclear. In a mechanistic study, Davis and coworkers ruled out the reduction hypothesis by UV-Vis and XANES (X-ray absorption near edge structure) spectroscopic studies of the catalyst before and after the HKR reaction [154]. Another suspected reason of deactivation, the formation of a catalyst dimer, was investigated by ESI-MS studies. While dimer formation was detected over time for Co-salen complex 64 (Figure 26) in dichloromethane, no difference in reactivity or enantioselectivity in the HKR reaction was observed between the dimeric material and fresh catalyst. Instead, it was proposed that the deactivation was mainly due to the formation of the less active $\mathrm{Co}(\mathrm{III})$ salen $(\mathrm{OH})$ species [155]. In the proposed mechanism (Scheme 45), the Co(III)salen $(\mathrm{OH})$ species is formed irreversibly and the more selective bimetallic pathway is dependent on the presence of both $\mathrm{Co}(\mathrm{III})$ salen $(\mathrm{OH})$ and $\mathrm{Co}(\mathrm{III})$ salen $(\mathrm{X})$, where the $\mathrm{Co}(\mathrm{III})$ salen $(\mathrm{X})$ complex is good at activating the epoxide. This hypothesis was further supported by experiments where a Co(III)salen $(\mathrm{Cl})$ complex was recycled in the HKR of epichlorohydrin. Without reactivation, $\mathrm{Co}(\mathrm{III})$ salen(Cl) showed a significant loss of catalytic activity after one reaction cycle, because of the rapid formation of $\mathrm{Co}(\mathrm{III}) \mathrm{salen}(\mathrm{OH})$. By adding a non-cooperative complex $\mathrm{Co}(\mathrm{III}) \operatorname{salen}\left(\mathrm{SbF}_{6}\right)$, the catalytic activity was completely restored.

There has also been some theoretical studies focused on the mechanistic origin of the high selectivity and broad scope of the HKR of epoxides catalyzed by Co(III)salen complexes. Jacobsen and coworkers published a comprehensive study on this issue, resulting in several important findings [156]. First, effective catalysis could be induced only when the two salen moieties in the cooperative pathway had the same absolute configuration. The specific stereochemistry of the salen was not important, only the matched stereochemistry (i.e., the two interacting Co-complexes should either both be $(S, S)$ or both $(R, R))$. Secondly, the stereochemical communication was primarily due to the stepped conformation of the entire metal-salen complex (Figure 50), and not due to the shape of the diamine backbone. The stepped conformation is the tilt of the salicylaldimine aryl rings relative to the equatorial plane of the complex. This conformation is not dependent on the presence of an enantiomerically pure diamine backbone that enforces the conformation, such as trans-1,2-diaminocyclohexyl. Rather, it was found that salen complexes derived from achiral diamines such as 1,2-ethylenediamine were also capable of adopting this stepped conformation. This finding was further supported by $\mathrm{X}$-ray crystal structures and computational studies. Finally, the binding geometry of the terminal epoxides to the Co(III)salen complexes was independent of the substituent of the epoxides which could explain the broad scope of HKR reactions catalyzed by $\mathrm{Co}(\mathrm{III})$ salen catalysts.

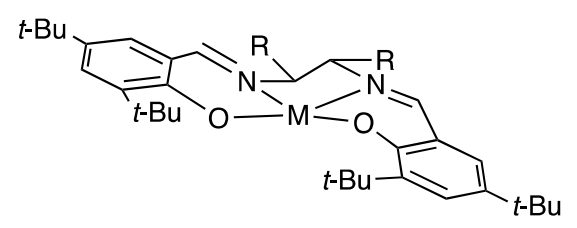

Figure 50. The stepped conformation of a metal-salen complex.

\subsubsection{Other Studies}

Berkessel and Ertuerk [157] reported an interesting catalyst for the HKR of terminal epoxides. They designed and synthesized DIANANE (endo,endo-2,5-diaminonorbornane)-based Cr(III) complexes 127 and 128 (Scheme 46), which exhibited high reactivity and enantioselectivity in the HKR of terminal epoxides under low catalyst loading (Scheme 46). Both catalysts were more enantioselective than the parent $\mathrm{Cr}$-salen complex $\mathbf{1}$ (Scheme 4). Complex $\mathbf{1 2 7}$ with $\mathrm{Cl}^{-}$counterion was less active than complex 128 with $\mathrm{OTs}^{-}$counterion, which is in line with the counterion effect previously described. 

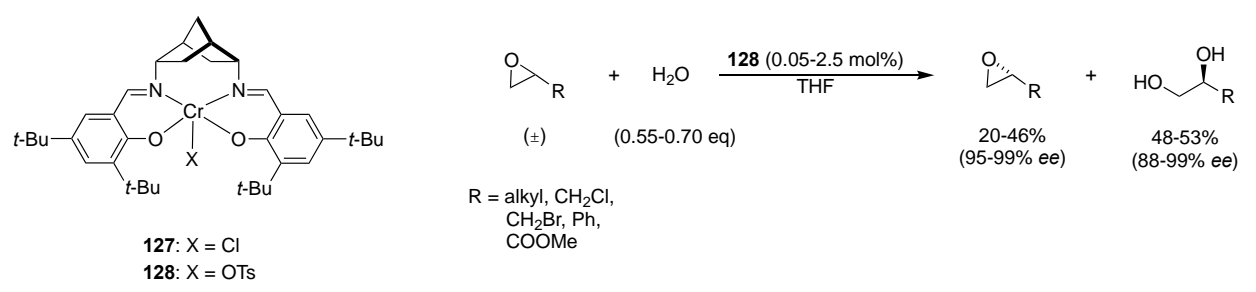

Scheme 46. The hydrolytic kinetic resolution of terminal epoxides catalyzed by Cr-DIANANE-salen complex 128. DIANANE $=$ endo,endo-2,5-diaminonorbornane.

Schulz and coworkers [67] implemented a heterobimetallic dual-catalyst system for the HKR of terminal epoxides. A 1:1 mixture of complex $\mathbf{1 2 9}$ and $\mathbf{1 3 0}$ (Figure 51) was employed in the HKR of phenyl glycidyl ether, allyl glycidyl ether, and styrene oxide in THF. The reactions with mixed catalysts afforded both products with excellent conversions (up to 57\%) and enantioselectivities (up to $94 \%$ ee for 1,2-diols, up to $99 \%$ ee for the unreacted epoxides), while reactions employing only catalyst 129 were less reactive and enantioselective. Kinetic studies revealed a first order dependence on the concentration of the Co-salen complex 129, which supported a heterobimetallic cooperative pathway similar to the cooperative mechanism in Scheme 45, where Co-salen complex 129 would be responsible for the generation of the nucleophilic $\mathrm{Co}(\mathrm{III}) \operatorname{salen}(\mathrm{OH})$ and $\mathrm{Mn}-$ salen complex 130 would be responsible for the activation of the epoxides. The study also included an investigation of different combinations of the absolute configuration of the two complexes. Higher enantioselectivity was achieved when the two complexes had the same absolute configuration which was in agreement with Jacobsen's [156] findings for a similar system.

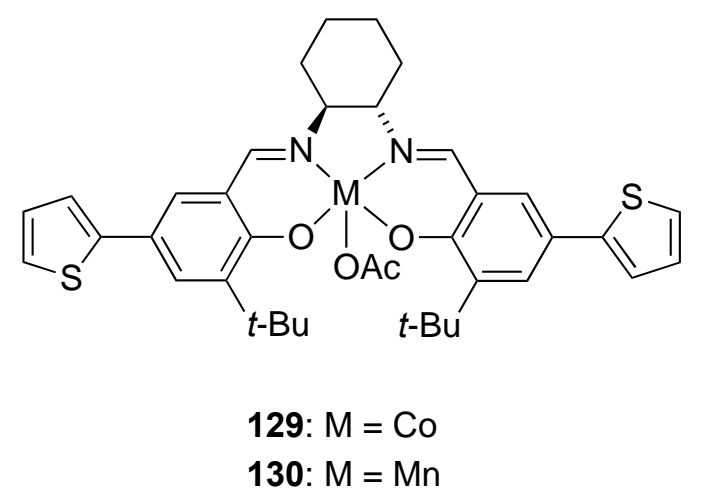

Figure 51. Salen complexes 130 and 131.

There are also examples of research that focus on more general reaction conditions. For example, Kim and coworkers [158] described the advantage of ultrasonication over mechanical stirring in the $\mathrm{Co}$ (III)salen-catalyzed HKR of terminal epoxides, both for homogeneous and heterogeneous catalysts. With ultrasonication, a shortening of the reaction time was observed in all cases.

\subsection{With Alcohols, Phenols, and Carboxylic Acids}

In addition to water (HKR), other oxygen-containing nucleophiles such as phenols, alcohols and carboxylic acids have also been employed in the KR of terminal epoxides. The ring-opened products are monoprotected enantioenriched 1,2-diols, which are versatile building blocks in the pharmaceutical industry and natural product synthesis [159]. Many of the catalysts mentioned above that are effective in the HKR of terminal epoxides are also found to work well for the KR of epoxides with phenols and alcohols as nucleophiles.

Jacobsen and coworkers [160] investigated several of their Co-salen complexes as catalysts in the KR of epoxides with phenols and alcohols as nucleophiles. In an early example, they employed catalyst 131 (Scheme 47) in the phenolic kinetic resolution of a number of different terminal epoxides. 
The ring-opened products where obtained in high yields and with high enantioselectivity (Scheme 47). The protocol could also be extended to different para- and meta-substituted phenols as nucleophiles, with consistently high yields and ee values for the ring-opened products.

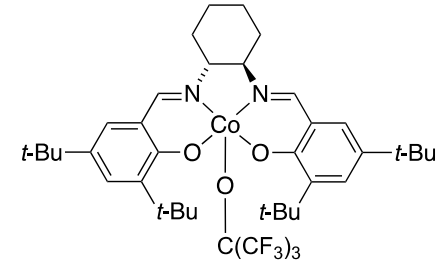

131

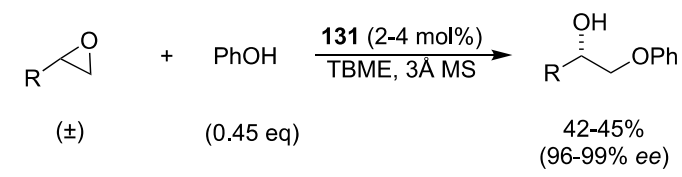

$\mathrm{R}=n-\mathrm{Bu}, \mathrm{CH}_{2} \mathrm{Cl}$,

$\mathrm{CH}_{2} \mathrm{O}$ (allyl), $\mathrm{c}$-Hex,

$\mathrm{C}(\mathrm{O}) \mathrm{CH}_{2} \mathrm{CH}_{3}, \mathrm{CO}_{2} \mathrm{CH}_{3}$

Scheme 47. The phenolic kinetic resolution catalyzed by Co-salen complex 131.

The polystyrene resin-supported Co-salen catalyst 108 (Figure 41) was employed in the phenolic $\mathrm{KR}$ of terminal epoxides in the first examples of an enantioselective catalytic synthesis of parallel libraries [161]. Following this protocol, a wide range of terminal epoxides and phenol nucleophiles were employed in parallel syntheses, resulting in 110 different 1-aryloxy-2-alcohols which were obtained in high yields (80-99\%) and $e e$ values (up to $99 \% e e$ ). The reactions were performed using the epoxide as a solvent. As such, an excess of epoxides was used and therefore all yields were calculated based on the nucleophile $[139,161]$.

The oligomeric macrocyclic Co-salen catalyst 35 (Scheme 10) was proven to also be efficient in the KR of epoxides with phenols and alcohols as nucleophiles [58]. Using complex $\mathbf{3 5}$ as catalyst, a broad range of phenols (with electron withdrawing and -donating substituents in the ortho-, metaand para-position) and primary alcohols (including benzylic and allylic) could be used as nucleophiles in the HKR of a number of different terminal epoxides. The ring-opened products were isolated in high yields (36-45\%) and with excellent enantioselectivities (97-99\% ee). All reactions were performed in acetonitrile with catalyst loadings of $0.0075-0.5 \mathrm{~mol} \% \mathrm{Co}$, with more sterically hindered nucleophiles requiring the higher catalyst loadings.

Weck and coworkers applied their oligomeric Co-salen complex 77 (Figure 31) in the KR of epichlorohydrin and 1,2-epoxyhexane with a limited number of phenols and aliphatic alcohols. The reactions performed in TBME or acetonitrile and with a low catalyst loading $(0.02 \mathrm{~mol} \% \mathrm{Co})$ afforded the ring-opened products in 38-45\% yield and 95-99\% ee [162].

Kim and coworkers published a number of studies where different $\mathrm{Co}(\mathrm{III})$ salen catalysts were employed in the phenolic KR of terminal epoxides. Most of these studies focused on dimeric Co-salen complexes linked by group 13 elements (catalyst $\mathbf{5 4 - 5 6}$ in Scheme 34) $[163,164]$ and the immobilization of these catalysts on microporous materials such as zeolite $[165,166]$, mesoporous materials such as SBA-15 and SBA-16 [146,148], and different macroporous materials [167,168]. In all cases the catalysts exhibited good enantioselectivities, but the substrate scopes were usually limited.

Kim's group also made efforts to apply Co-salen catalysts in the KR of terminal epoxides with carboxylic acids as nucleophiles. Dimeric $\mathrm{Co}$-salen complexes bridged by the Lewis acids $\mathrm{GaCl}_{3}$ and $\mathrm{Al}\left(\mathrm{NO}_{3}\right)_{3}$ (complex 55 in Scheme 34 and complex 132 in Figure 52) were investigated in the KR of terminal epoxides with a number of different carboxylic acids in TBME $[128,169]$. The reactions afforded 2-hydroxy monoesters in good yields (35-43\%) and with moderate to good enantioselectivities (53-86\% $e e)$, but most of the products needed further recrystallization or other treatment to provide practically applicable enantioenriched compounds. One of the applications of this reaction was the synthesis of highly enantioenriched (S)-glycidyl butyrate (Scheme 48). Hence, catalyst 55 was employed in the KR of epichlorohydrin with butyric acid. The so formed chlorohydrin $(76 \% e e)$ was then subjected to a ring closing reaction under basic conditions, again promoted by catalyst 55 , affording $(S)$-glycidyl butyrate in $98 \%$ ee. 


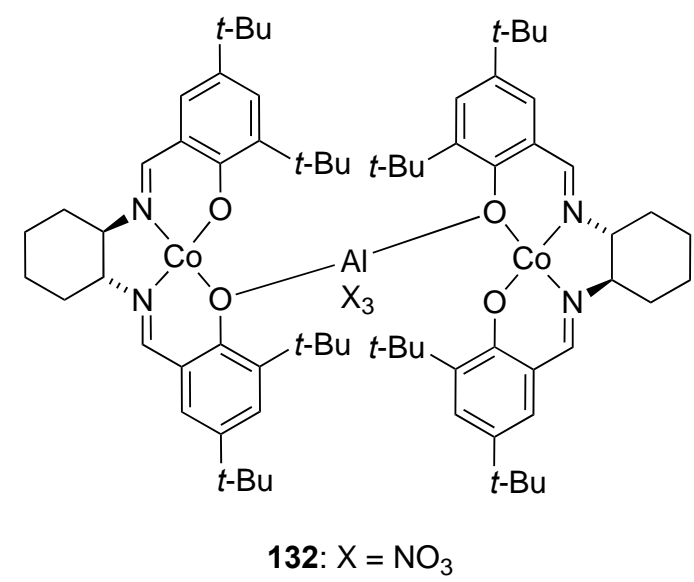

Figure 52. Dimeric Co-salen complex 132.

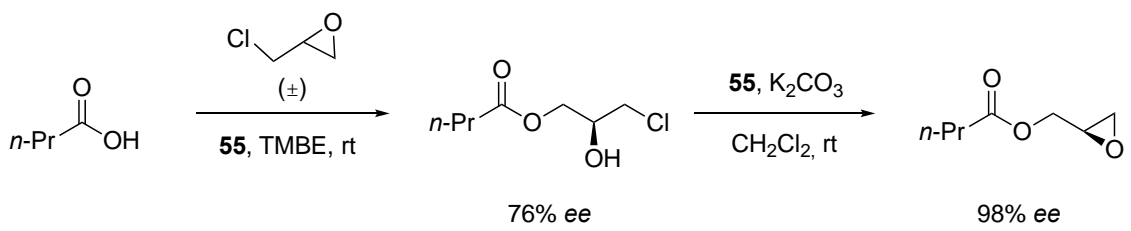

Scheme 48. The synthesis of (S)-glycidyl butyrate by kinetic resolution of epochlorohydrin employing catalyst 55 (Scheme 34).

The dimeric Co-salen catalyst 54 bridged by $\mathrm{AlCl}_{3}$ (Scheme 34) exhibited good catalytic activity in the KR of terminal epoxides with different sulfonic acids (Scheme 49) [170]. The reactions were performed in TBME and it was discovered that the addition of tetrabutylammonium chloride (TBACl) increased the yields and $e e$ values significantly (from $32 \%$ to $47 \%$ yield and from $68 \%$ to $99 \%$ ee for the reaction of phenyl glycidyl ether and $p$-toluenesulfonic acid). No explanation for this enhancement of the catalytic activity was given.

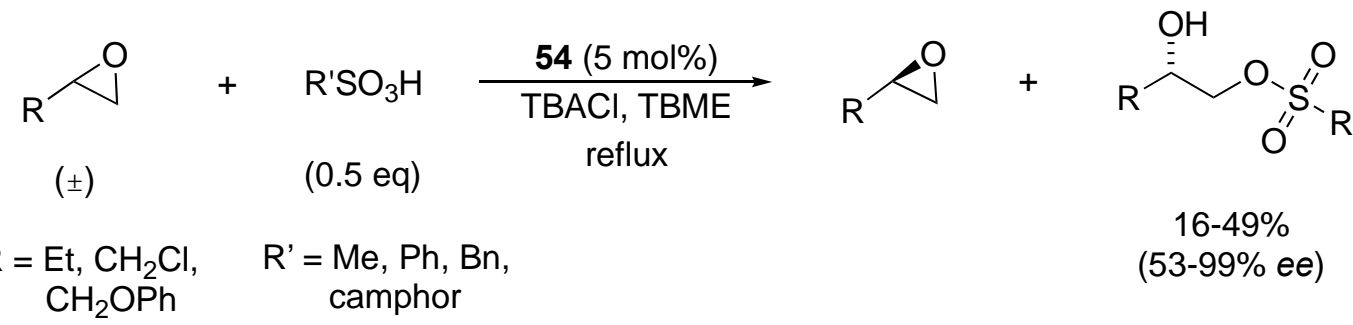

Scheme 49. The kinetic resolution of terminal epoxides with substituted sulfonic acid catalyzed by dimeric Co-salen catalyst 54 (Scheme 34). $\mathrm{TBACl}$ = tetrabutylammonium chloride.

\subsection{With Halogens}

Haufe and coworker investigated $\mathrm{Cr}$-salen complex 46 (Scheme 18) as catalyst for the enantioselective fluorination of epoxides [75-77]. The studies included a limited number of examples of the KR of terminal epoxides. The reaction required a very high catalyst loading $(50 \mathrm{~mol} \%)$ and the ring-opened products were obtained in moderate yield (28-29\%) and moderate to high enantioselectivity $(62-90 \%$ ee).

A more recent example of the enantioselective fluorination of epoxide was reported by Kalow and Doyle [79]. Using dimeric Co(II)salen complex 48 (Figure 21) together with cocatalyst 1,8-diazabicyclo[5.4.0]undec-7-ene (DBU) and HF (formed in situ from benzoyl fluoride and HFIP), the ring-opened products of a number of terminal epoxides were obtained in high yields and with excellent enantioselectivities (Scheme 50). The dimeric catalyst also showed a significant rate enhancement compared to the monomeric analogue and reactions were completed in 2-4 h. 


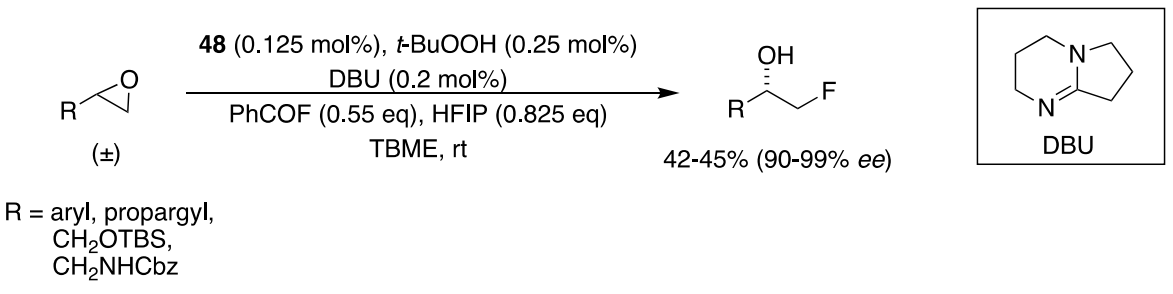

Scheme 50. The kinetic resolution of terminal epoxides with fluoride catalyzed by Co-salen complex 48 (Figure 21).

Kim and coworkers reported the $\mathrm{KR}$ of terminal epoxides with $\mathrm{HCl}$ catalyzed by dimeric $\mathrm{Co}$ (II)salen complexes linked by Lewis acids of group 13 metals (catalysts 54-56, Scheme 34). Catalyst 55 afforded both ring-opened products and unreacted epoxides in high yields and enantioselectivities (up to $90 \%$ ee and $89 \%$ ee, respectively) for terminal alkyl epoxides and glycidyl ethers (Scheme 51). Aryl glycidyl ethers gave significantly lower $e e$ values than alkyl glycidyl ethers and terminal alkyl epoxides. It was discovered that the $e e$ value of the unreacted epoxide decreased over time, as the formed chlorohydrin was capable of performing the reverse ring-closing reaction in the presence of catalyst 55. To prevent this, the reaction had to be terminated once a high ee value was reached for the unreacted epoxide. Different group 13 metals and anions were evaluated but showed similar reactivities and enantioselectivities [127,128].

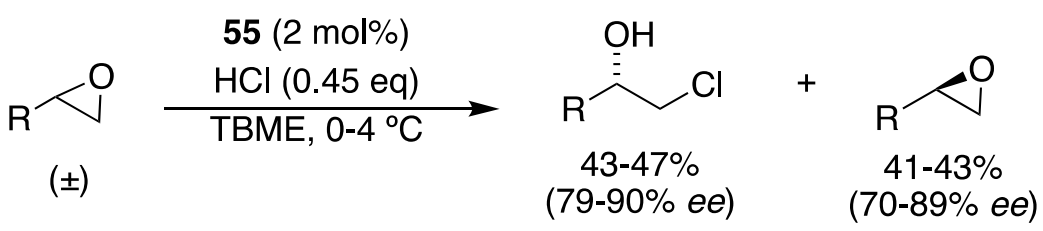

Scheme 51. The kinetic resolution of terminal epoxides with hydrochloric acid catalyzed by dimeric Co-salen complex 55 (Scheme 34).

\subsection{With Carbon-Containing Nucleophiles}

Cozzi and Umani-Ronchi [90] used Cr-salen-SbF 6 complex 133 (Figure 53) as a catalyst for the $\mathrm{KR}$ of 1,2-disubstituted aromatic epoxides with indoles. The ring-opened products were obtained in high yields and with high enantioselectivities (Scheme 52). Notably, this constitutes a rare example of a reported method for the KR of epoxides that is efficient for both cis and trans aromatic epoxides. For all substrates, the ring-opened products were obtained with complete regioselectivity (nucleophilic attack at the benzylic carbon of the epoxides). Moreover, by adjusting the amount of indole used, the reaction could be tuned to afford unreacted epoxides in satisfactory yields and with excellent enantioselectivities (91-99\% ee).

Recently, Hajra and Roy [171] reported the first enantioselective construction of an all-carbon quaternary center from an epoxide by KR, using Co(III)salen catalysts 73 (Figure 29) and $\mathbf{1 3 4}$ (Figure 54) and spiro-epoxides with $N$-benzylindoles as nucleophiles. The desired 3,3'-bisindole methanols were obtained with complete regioselectivity, that is, with reaction of the nucleophile at the more substituted carbon of the epoxide. The excellent regioselectivity of the reaction was explained by a proposed mechanism where the Lewis acid coordinating to the epoxyindole led to the ring-opening of the epoxide to the more stabilized tertiary carbocation before the nucleophile attacks [172]. Under optimized conditions with $\mathrm{Co}(\mathrm{III})$ salen(OTf) catalyst 73, which included the addition of 1 equivalent of water, the ring-opened products were obtained in good yields and with high enantioselectivities. The unreacted epoxides were recovered in low-to-moderate enantioselectivities. Using $\mathrm{Co}(\mathrm{III}) \mathrm{salen}\left(\mathrm{SbF}_{6}\right)$ complex $\mathbf{1 3 4}$ as catalyst resulted in a DKR, giving ring-opened products in good yields and with moderate-to-good enantioselectivities, with no unreacted epoxides recovered (Scheme 53). Mechanistic studies indicated that the ring-opening reaction of spiro-epoxides was governed by an equilibration between KR and DKR 
processes, and that this equilibrium was further controlled by feedback inhibition caused by the formation of a catalyst-product complex. Catalyst 134 with a non-coordinating counterion was less influenced by the feedback inhibition and could therefore be operated by the dynamic kinetic process [171].

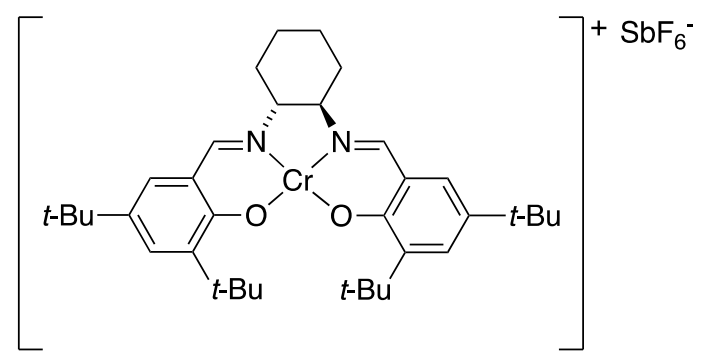

133

Figure 53. Cr-salen complex 133.

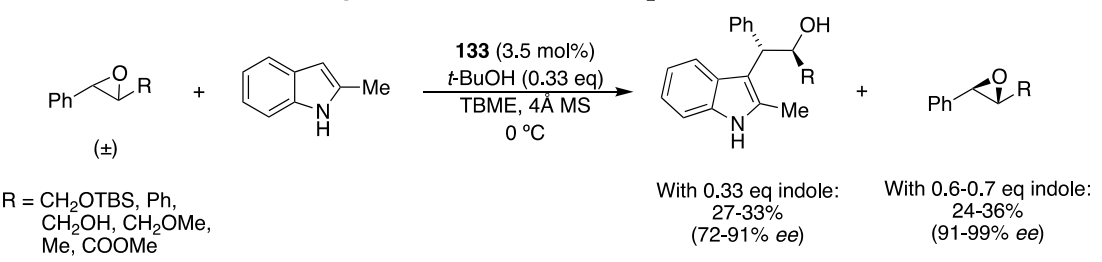

Scheme 52. The kinetic resolution of internal 1,2-disubstituted aromatic epoxides with indoles catalyzed by Cr-salen complex 133 (Figure 53).<smiles></smiles>

134

Figure 54. Co(II)salen complex 134.

(a)<smiles>[R][R]1ccc2cc(Br)n(Br)c2c1</smiles>

$( \pm)$

\section{$73(10 \mathrm{~mol} \%)$} $\frac{\mathrm{H}_{2} \mathrm{O}(1 \mathrm{eq})}{\mathrm{C}_{2} \mathrm{H}_{2} \mathrm{Cl}_{2}, \mathrm{rt}}$

KR<smiles>c1ccc(Cn2ccc3ccccc32)cc1</smiles>

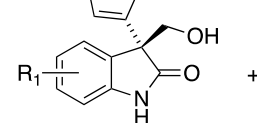

$38-45 \%(85-96 \%$ ee)

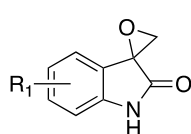

$(7-70 \%$ ee)

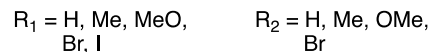

(b)

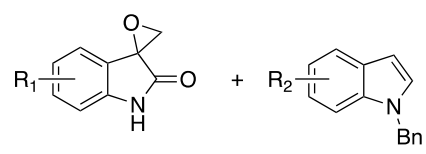

$( \pm)$

(1.5 eq)

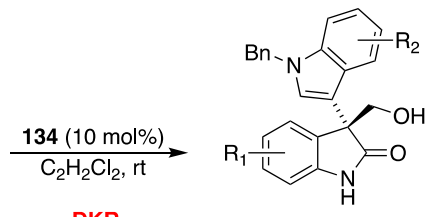

$\mathrm{R}_{1}=\mathrm{H}, \mathrm{Me}, \mathrm{MeO}$

$\mathrm{R}_{2}=\mathrm{H}, \mathrm{Me}$

Scheme 53. (a) The kinetic resolution (KR) of spiro-epoxides with $N$-benzylindoles catalyzed by Co-salen complex 73 (Figure 29). (b) The dynamic kinetic resolution (DKR) of spiro-epoxides with $\mathrm{N}$-benzylindoles catalyzed by Co-salen complexes 134 (Figure 54). Both processes generate quaternary all-carbon centers with high enantioselectivity. 


\section{Conclusions and Outlook}

The catalytic asymmetric ring-opening of epoxides is a highly useful method for the preparation of synthetically important, vicinally difunctionalized organic compounds such as amino alcohols, diols, and halohydrins in high yield and with high enantiomeric purity. The use of metal-salen complexes as catalysts for ARO reactions has seen much development since the first reports some 25 years ago. From an enantioselectivity standpoint, the original monosalen catalysts reported by Jacobsen (Cr-salen for ARO with azides, Scheme 4, and Co-salen for ARO with water, Scheme 40) remain competitive to this day, but significant progress has been made in terms of catalyst loading, substrate scope, and recyclability.

One area of interest has been the development of multi-metallic catalysts capable of enforcing a bimetallic cooperative pathway. Numerous strategies have been employed, ranging from covalently linking two or more salen complexes together by installing them on dendrimers, polymers or in supramolecular assemblies. Although this approach often requires more laborious synthetic efforts, many of the resulting catalysts have shown significantly increased reaction rates for the ARO of epoxides compared to monosalen analogues, and also enabled efficient catalysis at considerably lower catalyst loadings. In addition, the development and application of multi-metallic complexes have provided further insight into the reaction mechanism and highlighted the importance of factors such as the orientation of the complexes relative to each other as well as the length and flexibility of bridging linkers.

As large-scale applications depend largely on the separation of the products and recovery and recycling of the catalyst, the development of heterogeneous catalysts has received much attention. Metal-salen complexes have been immobilized on a number of different solid surfaces and materials, providing access to catalysts with good stability that can be easily recycled without loss of catalytic properties. A further challenge in this area is to design the catalyst so that the cooperative interactions are still possible in order to achieve high reactivity and enantioselectivity. In terms of recyclability, the use of ionic liquids has also received some attention and shows much promise. Looking forward, one interesting area that has so far only been sparingly explored is the development of catalysts bridging homogeneous and heterogeneous catalysis, for example, by using soluble supports or encapsulating metal-salen complexes inside porous materials. The facile synthesis and easy modification of metal-salen complexes has also made them attractive catalysts to use for demonstrating the design and efficacy of new catalytic concepts and systems in general.

Different catalytic systems based on increasing the local concentration of catalyst or improving the cooperative interactions have allowed for significantly decreased catalyst loadings. This includes the development of polymeric salen complexes and the incorporation of metal-salen complexes in nanoreactors. Very low catalyst loadings $(<0.01 \mathrm{~mol} \%)$ have mainly been realized for the hydrolytic kinetic resolution (HKR) of epoxides catalyzed by Co-salen complexes, with a few examples for the ARO of meso-epoxides with azides catalyzed by $\mathrm{Cr}$-salen. However, for other nucleophiles there is still a lot of room for improvement.

Regarding the substrate scope, many of the reported catalysts have only been investigated for a limited number of substrates, both in terms of nucleophiles and epoxides. With regard to the nucleophile, one desirable development would be a wider application and investigation of different carbon-based nucleophiles in the metal-salen catalyzed ARO of epoxides, in order to produce asymmetric $\mathrm{C}-\mathrm{C}$ bonds. Another class of nucleophiles that merits further investigation is oxygen-containing nucleophiles other than water, as this could result in the regioselective preparation of monoprotected diols, something which can be difficult to achieve by other methods.

Another aspect of the substrate scope is the epoxide. For the KR of epoxides, the vast majority of the research has been focused on terminal epoxides. This can be explained by the limited availability of enantiopure terminal epoxides by other methods, making the KR of terminal epoxides highly attractive as the unreacted epoxide can be recovered in high ee. As such, the metal-salen-catalyzed HKR have been successfully applied to a broad range of terminal epoxides with a wide variety of 
substituents, although no examples of the HKR of internal epoxides have been published to date. There are a few examples of the KR of trans-epoxides with anilines and indoles, and one example of the KR of cis-epoxides with indoles, but other than that there is a distinct lack of protocols for the KR of internal epoxides.

Extending the metal-salen catalyzed ARO of epoxides to include internal epoxides would also make it a good complement to other synthetic protocols. For example, the successful HKR of trans-epoxides would yield anti-diols, making it complementary to Sharpless dihydroxylation of olefins which works well for trans-olefins and gives the corresponding syn-diols with high regio-, diastereoand enantioselectivity [64]. In addition, since asymmetric epoxidation methods such as Jacobsen's Mn-salen epoxidation usually work poorly for trans-alkenes [24,173], achieving an efficient KR of trans-epoxides would give access to otherwise difficult to obtain enantiopure trans-epoxides.

There is also a lack of examples of the ARO of more substituted epoxides, with only a handful reported examples of the ARO of trisubstituted and 2,2-disubstituted epoxides. Extending the substrate scope to more substituted epoxides would also enable further studies of the regioselectivity of this type of catalysis. So far, most studies report complete regioselectivity with the investigated substrate scope, but further studies are needed to better understand, and by extension tune, this selectivity.

There are also several examples of innovative ways of utilizing metal-salen-catalyzed ARO reactions to solve general challenging synthetic problems. This includes the enantioselective construction of all-carbon quaternary centers from epoxides and the development of sequential asymmetric alkene epoxidation/ring-opening reactions. Both of these reactions are potentially of high importance and we hope to see much further research in this direction. Another aspect that merits further study is the dynamic kinetic resolution, which allows the preparation of enantiopure ring-opened products from racemic starting materials in $100 \%$ theoretical yield. There are a few examples of the successful application of this highly attractive strategy, mainly for the HKR of terminal epoxides. However, it is an area where much progress remains to be made.

To conclude, catalytic systems for the asymmetric ring opening of epoxides based on metal-salen complexes have been extensively studied and developed. With excellent catalytic activity and selectivity, this class of catalysts is a valuable tool in the area of asymmetric catalysis. As such, it remains an active field of research and we expect further advances are still forthcoming.

Author Contributions: Conceptualization, A.L., Y.L. and K.W.; writing—original draft preparation, A.L. and Y.L.; writing-review and editing, A.L., Y.L. and K.W. All authors have read and agreed to the published version of the manuscript.

Funding: K.W. thanks the Swedish Research Council, the LMK foundation, the Swedish Foundation for Strategic Research, and the Knut and Alice Wallenbergs Stiftelse for generous grants. Y.L. thanks the Chinese Scholarship Council for the scholarship.

Conflicts of Interest: The authors declare no conflict of interest.

\section{References}

1. Walsh, P.J.; Kozlowski, M.C. Fundamentals of Asymmetric Catalysis; University Science Books: Sausalito, CA, USA, 2009.

2. Halpern, J.; Trost, B.M. Asymmetric Catalysis. Proc. Natl. Acad. Sci. USA 2004, 101, 5347. [CrossRef]

3. Yoon, T.P.; Jacobsen, E.N. Privileged Chiral Catalysts. Science 2003, 299, 1691. [CrossRef]

4. Shaw, S.; White, J.D. Asymmetric Catalysis Using Chiral Salen-Metal Complexes: Recent Advances. Chem. Rev. 2019, 119, 9381-9426. [CrossRef]

5. Cozzi, P.G. Metal-Salen Schiff base complexes in catalysis: Practical aspects. Chem. Soc. Rev. 2004, 33, $410-421$. [CrossRef]

6. Zhang, W.-Z.; Lu, X.-B. Chiral Salen Complexes. In Privileged Chiral Ligands and Catalysts; Zhou, Q.L., Ed.; Wiley: Weinheim, Germany, 2011; pp. 257-293.

7. Trost, B.; Fleming, I. Comprehensive Organic Synthesis; Pergamon: Oxford, UK, 1991. 
8. Xia, Q.H.; Ge, H.Q.; Ye, C.P.; Liu, Z.M.; Su, K.X. Advances in Homogeneous and Heterogeneous Catalytic Asymmetric Epoxidation. Chem. Rev. 2005, 105, 1603-1662. [CrossRef]

9. Pastor, I.M.; Yus, M. Asymmetric Ring Opening of Epoxides. Curr. Org. Chem. 2005, 9, 1-29. [CrossRef]

10. Schneider, C. Synthesis of 1, 2-difunctionalized fine chemicals through catalytic, enantioselective ring-opening reactions of epoxides. Synthesis 2006, 2006, 3919-3944. [CrossRef]

11. Clarke, R.M.; Storr, T. The chemistry and applications of multimetallic salen complexes. Dalton Trans. 2014, 43, 9380-9391. [CrossRef]

12. Matsunaga, S.; Shibasaki, M. Multimetallic Schiff Base Complexes as Cooperative Asymmetric Catalysts. Synthesis 2013, 45, 421-437. [CrossRef]

13. Haak, R.M.; Wezenberg, S.J.; Kleij, A.W. Cooperative multimetallic catalysis using metallosalens. Chem. Commun. 2010, 46, 2713-2723. [CrossRef]

14. Abd El Sater, M.; Jaber, N.; Schulz, E. Chiral Salen Complexes for Asymmetric Heterogeneous Catalysis: Recent Examples for Recycling and Cooperativity. Chem CatChem 2019, 11, 3662-3687. [CrossRef]

15. Baleizão, C.; Garcia, H. Chiral Salen Complexes: An Overview to Recoverable and Reusable Homogeneous and Heterogeneous Catalysts. Chem. Rev. 2006, 106, 3987-4043. [CrossRef]

16. Shioiri, T.; Hamada, Y. Natural product syntheses utilizing 4-alkoxycarbonyloxazoles as $\beta$-hydroxy- $\alpha$-amino acid synthons. Heterocycles 1988, 27, 1035-1050. [CrossRef]

17. Kikelj, D.; Kidrič, J.; Pristovšek, P.; Pečar, S.; Urleb, U.; Krbavčič, A.; Hönig, H. Preparation of diastereomerically pure immunologically active carbocyclic nor-muramyldipeptide analogues. Tetrahedron 1992, 48, 5915-5932. [CrossRef]

18. Vacca, J.P.; Dorsey, B.; Schleif, W.; Levin, R.; McDaniel, S.; Darke, P.; Zugay, J.; Quintero, J.; Blahy, O.; Roth, E. L-735,524: An orally bioavailable human immunodeficiency virus type 1 protease inhibitor. Proc. Natl. Acad. Sci. USA 1994, 91, 4096-4100. [CrossRef]

19. Wang, R.; Wong, C.-H. Synthesis of sialyl Lewis X mimetics: Use of O- $\alpha$-fucosyl-(1R, 2R)-2-aminocyclohexanol as core structure. Tetrahedron Lett. 1996, 37, 5427-5430. [CrossRef]

20. Martinez, L.E.; Leighton, J.L.; Carsten, D.H.; Jacobsen, E.N. Highly enantioselective ring opening of epoxides catalyzed by (salen) Cr (III) complexes. J. Am. Chem. Soc. 1995, 117, 5897-5898. [CrossRef]

21. Jacobsen, E.N. Asymmetric catalysis of epoxide ring-opening reactions. Acc. Chem. Res. 2000, 33, $421-431$. [CrossRef]

22. Pakulski, Z.; Pietrusiewicz, K.M. Enantioselective desymmetrization of phospholene meso-epoxide by nucleophilic opening of the epoxide. Tetrahedron Asymmetry 2004, 15, 41-45. [CrossRef]

23. Saha, B.; Lin, M.-H.; RajanBabu, T. Exceptionally Active Yttrium-Salen Complexes for the Catalyzed Ring Opening of Epoxides by TMSCN and TMSN3. J. Org. Chem. 2007, 72, 8648-8655. [CrossRef]

24. Zhang, W.; Loebach, J.L.; Wilson, S.R.; Jacobsen, E.N. Enantioselective epoxidation of unfunctionalized olefins catalyzed by salen manganese complexes. J. Am. Chem. Soc. 1990, 112, 2801-2803. [CrossRef]

25. Jacobsen, E.N.; Zhang, W.; Muci, A.R.; Ecker, J.R.; Deng, L. Highly enantioselective epoxidation catalysts derived from 1, 2-diaminocyclohexane. J. Am. Chem. Soc. 1991, 113, 7063-7064. [CrossRef]

26. Brandes, B.D.; Jacobsen, E.N. Highly enantioselective, catalytic epoxidation of trisubstituted olefins. J. Org. Chem. 1994, 59, 4378-4380. [CrossRef]

27. Schaus, S.E.; Larrow, J.F.; Jacobsen, E.N. Practical synthesis of enantiopure cyclic 1, 2-amino alcohols via catalytic asymmetric ring opening of meso epoxides. J. Org. Chem. 1997, 62, 4197-4199. [CrossRef]

28. Hansen, K.B.; Leighton, J.L.; Jacobsen, E.N. On the mechanism of asymmetric nucleophilic ring-opening of epoxides catalyzed by (salen) CrIII complexes. J. Am. Chem. Soc. 1996, 118, 10924-10925. [CrossRef]

29. Guillaneux, D.; Zhao, S.-H.; Samuel, O.; Rainford, D.; Kagan, H.B. Nonlinear effects in asymmetric catalysis. J. Am. Chem. Soc. 1994, 116, 9430-9439. [CrossRef]

30. Konsler, R.G.; Karl, J.; Jacobsen, E.N. Cooperative asymmetric catalysis with dimeric salen complexes. J. Am. Chem. Soc. 1998, 120, 10780-10781. [CrossRef]

31. Ma, D.Y.; Xiao, Z.Y.; Etxabe, J.; Wärnmark, K. Pseudo-C2-Symmetric Bimetallic Bissalen Catalysts for Efficient and Enantioselective Ring-Opening of meso-Epoxides. ChemCatChem 2012, 4, 1321-1329. [CrossRef]

32. Li, Y.; Lidskog, A.; Armengol-Relats, H.; Pham, T.H.; Favraud, A.; Nicolas, M.; Dawaigher, S.; Xiao, Z.; Ma, D.; Lindbäck, E.; et al. Enantiotopic Discrimination by Coordination-Desymmetrized meso-Ligands. ChemCatChem 2020, 12, 1575-1579. [CrossRef] 
33. Gianneschi, N.C.; Bertin, P.A.; Nguyen, S.T.; Mirkin, C.A.; Zakharov, L.N.; Rheingold, A.L. A supramolecular approach to an allosteric catalyst. J. Am. Chem. Soc. 2003, 125, 10508-10509. [CrossRef]

34. Gianneschi, N.C.; Cho, S.H.; Nguyen, S.T.; Mirkin, C.A. Reversibly addressing an allosteric catalyst in situ: Catalytic molecular tweezers. Angew. Chem. Int. Ed. 2004, 43, 5503-5507. [CrossRef] [PubMed]

35. Ma, D.Y.; Norouzi-Arasi, H.; Sheibani, E.; Wärnmark, K. Dynamic Supramolecular [(Salen) CrCl] Complexes as Efficient Catalysts for Ring Opening of Epoxides. Chem CatChem 2010, 2, 629-632. [CrossRef]

36. Lindbäck, E.; Norouzi-Arasi, H.; Sheibani, E.; Ma, D.; Dawaigher, S.; Wärnmark, K. Synthesis of Cr (III) Salen Complexes as Supramolecular Catalytic Systems for Ring-Opening Reactions of Epoxides. ChemistrySelect 2016, 1, 1789-1794. [CrossRef]

37. Odille, F.G.J.; Jónsson, S.; Stjernqvist, S.; Rydén, T.; Wärnmark, K. On the Characterization of Dynamic Supramolecular Systems: A General Mathematical Association Model for Linear Supramolecular Copolymers and Application on a Complex Two-Component Hydrogen-Bonding System. Chem. Eur. J. 2007, 13, 9617-9636. [CrossRef] [PubMed]

38. Zulauf, A.; Mellah, M.; Schulz, E. New Chiral Calixsalen Chromium Complexes: Recyclable Asymmetric Catalysts. Chem. Eur. J. 2010, 16, 11108-11114. [CrossRef]

39. Canali, L.; Sherrington, D.C. Utilisation of homogeneous and supported chiral metal (salen) complexes in asymmetric catalysis. Chem. Soc. Rev. 1999, 28, 85-93. [CrossRef]

40. Angelino, M.D.; Laibinis, P.E. Polymer-supported salen complexes for heterogeneous asymmetric synthesis: Stability and selectivity. J. Polym. Sci. Part A Polym. Chem. 1999, 37, 3888-3898. [CrossRef]

41. Gigante, B.; Corma, A.; García, H.; Sabater, M.J. Assessment of the negative factors responsible for the decrease in the enantioselectivity for the ring opening of epoxides catalyzed by chiral supported $\mathrm{Cr}$ (III)-salen complexes. Catal. Lett. 2000, 68, 113-119. [CrossRef]

42. Baleizão, C.; Gigante, B.; Sabater, M.J.; Garcia, H.; Corma, A. On the activity of chiral chromium salen complexes covalently bound to solid silicates for the enantioselective epoxide ring opening. Appl. Catal. A 2002, 228, 279-288. [CrossRef]

43. Dioos, B.M.; Geurts, W.A.; Jacobs, P.A. Coordination of Cr III (salen) on functionalised silica for asymmetric ring opening reactions of epoxides. Catal. Lett. 2004, 97, 125-129. [CrossRef]

44. Dioos, B.M.L.; Jacobs, P.A. Impregnation of dimeric CrIII(salen) on silica and its application in epoxide asymmetric ring opening reactions. Appl. Catal. A 2005, 282, 181-188. [CrossRef]

45. Dioos, B.M.L.; Jacobs, P.A. CrIII(salen) impregnated on silica for asymmetric ring opening reactions and its recovery via desorption/re-impregnation. Tetrahedron Lett. 2003, 44, 8815-8817. [CrossRef]

46. Dioos, B.M.L.; Jacobs, P.A. Heterogenisation of dimeric $\mathrm{Cr}$ (salen) with supported ionic liquids. J. Catal. 2006, 243, 217-219. [CrossRef]

47. Keilitz, J.; Haag, R. Intramolecular Acceleration of Asymmetric Epoxide Ring-Opening by Dendritic Polyglycerol Salen-CrIII Complexes. Eur. J. Org. Chem. 2009, 2009, 3272-3278. [CrossRef]

48. Zheng, X.; Jones, C.W.; Weck, M. Ring-Expanding Olefin Metathesis: A Route to Highly Active Unsymmetrical Macrocyclic Oligomeric Co-Salen Catalysts for the Hydrolytic Kinetic Resolution of Epoxides. J. Am. Chem. Soc. 2007, 129, 1105-1112. [CrossRef]

49. Kinslow, K.; Sevde, A.M.; Liang, J.; Liu, Y. Enantioselective ring opening of epoxides with TMSN3 by macrocyclic oligomer-supported Cr (III)-salen catalysts. Tetrahedron Asymmetry 2015, 26, 385-392. [CrossRef]

50. Song, C.E.; Oh, C.R.; Roh, E.J.; Choo, D.J. Cr (salen) catalysed asymmetric ring opening reactions of epoxides in room temperature ionic liquids. Chem. Commun. 2000, 1743-1744. [CrossRef]

51. Jiao, J.; Tan, C.; Li, Z.; Liu, Y.; Han, X.; Cui, Y. Design and assembly of chiral coordination cages for asymmetric sequential reactions. J. Am. Chem. Soc. 2018, 140, 2251-2259. [CrossRef]

52. Tan, C.; Han, X.; Li, Z.; Liu, Y.; Cui, Y. Controlled exchange of achiral linkers with chiral linkers in Zr-based UiO-68 metal-organic framework. J. Am. Chem. Soc. 2018, 140, 16229-16236. [CrossRef]

53. Zhang, X.; Hou, Y.; Ettelaie, R.; Guan, R.; Zhang, M.; Zhang, Y.; Yang, H. Pickering Emulsion-Derived Liquid-Solid Hybrid Catalyst for Bridging Homogeneous and Heterogeneous Catalysis. J. Am. Chem. Soc. 2019, 141, 5220-5230. [CrossRef]

54. Bartoli, G.; Bosco, M.; Carlone, A.; Locatelli, M.; Massaccesi, M.; Melchiorre, P.; Sambri, L. Asymmetric Aminolysis of Aromatic Epoxides: A Facile Catalytic Enantioselective Synthesis of anti- $\beta$-Amino Alcohols. Org. Lett. 2004, 6, 2173-2176. [CrossRef] [PubMed] 
55. Kureshy, R.I.; Kumar, M.; Agrawal, S.; Khan, N.U.H.; Dangi, B.; Abdi, S.H.; Bajaj, H.C. Enantioselective desymmetrization of meso-epoxides with anilines catalyzed by polymeric and monomeric Ti (IV) salen complexes. Chirality 2011, 23, 76-83. [CrossRef]

56. Kureshy, R.I.; Prathap, K.J.; Kumar, M.; Bera, P.K.; Khan, N.-u.H.; Abdi, S.H.R.; Bajaj, H.C. Synthesis of enantiopure $\beta$-amino alcohols via AKR/ARO of epoxides using recyclable macrocyclic $\mathrm{Cr}$ (III) salen complexes. Tetrahedron 2011, 67, 8300-8307. [CrossRef]

57. Birrell, J.A.; Jacobsen, E.N. A practical method for the synthesis of highly enantioenriched trans-1, 2-amino alcohols. Org. Lett. 2013, 15, 2895-2897. [CrossRef] [PubMed]

58. White, D.E.; Tadross, P.M.; Lu, Z.; Jacobsen, E.N. A broadly applicable and practical oligomeric (salen)Co catalyst for enantioselective epoxide ring-opening reactions. Tetrahedron 2014, 70, 4165-4180. [CrossRef] [PubMed]

59. Sharma, A.; Agarwal, J.; Peddinti, R.K. Direct access to the optically active VAChT inhibitor vesamicol and its analogues via the asymmetric aminolysis of meso-epoxides with secondary aliphatic amines. Org. Biomol. Chem. 2017, 15, 1913-1920. [CrossRef]

60. Roy, S.; Bhanja, P.; Islam, S.S.; Bhaumik, A.; Islam, S.M. A new chiral Fe (iii)-salen grafted mesoporous catalyst for enantioselective asymmetric ring opening of racemic epoxides at room temperature under solvent-free conditions. Chem. Commun. 2016, 52, 1871-1874. [CrossRef]

61. Islam, M.M.; Bhanja, P.; Halder, M.; Kundu, S.K.; Bhaumik, A.; Islam, S.M. Chiral Co(iii)-salen complex supported over highly ordered functionalized mesoporous silica for enantioselective aminolysis of racemic epoxides. RSC Adv. 2016, 6, 109315-109321. [CrossRef]

62. Sun, Z.; Chen, J.; Liu, Y.; Tu, T. Chiral Titanium Coordination Assemblies: Robust Cooperative Self-Supported Catalysts for Asymmetric Ring Opening of meso-Epoxides with Aliphatic Amines. Adv. Synth. Catal. 2017, 359, 494-505. [CrossRef]

63. Xi, W.; Liu, Y.; Xia, Q.; Li, Z.; Cui, Y. Direct and Post-Synthesis Incorporation of Chiral Metallosalen Catalysts into Metal-Organic Frameworks for Asymmetric Organic Transformations. Chem. Eur. J. 2015, 21, 12581-12585. [CrossRef]

64. Kolb, H.C.; VanNieuwenhze, M.S.; Sharpless, K.B. Catalytic Asymmetric Dihydroxylation. Chem. Rev. 1994, 94, 2483-2547. [CrossRef]

65. Kim, G.-J.; Park, D.-W. The catalytic activity of new chiral salen complexes immobilized on MCM-41 in the asymmetric hydrolysis of epoxides to diols. Catal. Today 2000, 63, 537-547. [CrossRef]

66. Haak, R.M.; Martinez Belmonte, M.; Escudero-Adan, E.C.; Benet-Buchholz, J.; Kleij, A.W. Olefin metathesis as a tool for multinuclear Co(III)salen catalyst construction: Access to cooperative catalysts. Dalton Trans. 2010, 39, 593-602. [CrossRef] [PubMed]

67. Hong, X.; Mellah, M.; Schulz, E. Heterobimetallic dual-catalyst systems for the hydrolytic kinetic resolution of terminal epoxides. Catal. Sci. Technol. 2014, 4, 2608-2617. [CrossRef]

68. Dandachi, H.; Zaborova, E.; Kolodziej, E.; David, O.R.P.; Hannedouche, J.; Mellah, M.; Jaber, N.; Schulz, E. Mixing and matching chiral cobalt- and manganese-based calix-salen catalysts for the asymmetric hydrolytic ring opening of epoxides. Tetrahedron Asymmetry 2016, 27, 246-253. [CrossRef]

69. Ready, J.M.; Jacobsen, E.N. Highly active oligomeric (salen)Co catalysts for asymmetric epoxide ring-opening reactions. J. Am. Chem. Soc. 2001, 123, 2687-2688. [CrossRef]

70. Liang, J.; Soucie, L.N.; Blechschmidt, D.R.; Yoder, A.; Gustafson, A.; Liu, Y. Aromatic Donor-Acceptor Interaction-Based Co(III)-salen Self-Assemblies and Their Applications in Asymmetric Ring Opening of Epoxides. Org. Lett. 2019, 21, 513-518. [CrossRef]

71. Jacobsen, E.N.; Kakiuchi, F.; Konsler, R.G.; Larrow, J.F.; Tokunaga, M. Enantioselective catalytic ring opening of epoxides with carboxylic acids. Tetrahedron Lett. 1997, 38, 773-776. [CrossRef]

72. Müller, K.; Faeh, C.; Diederich, F. Fluorine in pharmaceuticals: Looking beyond intuition. Science 2007, 317, 1881-1886. [CrossRef]

73. Purser, S.; Moore, P.R.; Swallow, S.; Gouverneur, V. Fluorine in medicinal chemistry. Chem. Soc. Rev. 2008, 37, 320-330. [CrossRef]

74. Banks, R.E.; Smart, B.E.; Tatlow, J. Organofluorine Chemistry: Principles and Commercial Applications; Springer Science \& Business Media: New York, NY, USA, 2013.

75. Bruns, S.; Haufe, G. Enantioselective introduction of fluoride into organic compounds: First asymmetric ring opening of epoxides by hydrofluorinating reagents. J. Fluor. Chem. 2000, 104, 247-254. [CrossRef] 
76. Haufe, G.; Bruns, S.; Runge, M. Enantioselective ring-opening of epoxides by HF-reagents: Asymmetric synthesis of fluoro lactones. J. Fluor. Chem. 2001, 112, 55-61. [CrossRef]

77. Haufe, G.; Bruns, S. (Salen)chromium Complex Mediated Asymmetric Ring Opening of meso- and Racemic Epoxides with Different Fluoride Sources. Adv. Synth. Catal. 2002, 344, 165-171. [CrossRef]

78. Kalow, J.A.; Doyle, A.G. Enantioselective ring opening of epoxides by fluoride anion promoted by a cooperative dual-catalyst system. J. Am. Chem. Soc. 2010, 132, 3268-3269. [CrossRef]

79. Kalow, J.A.; Doyle, A.G. Mechanistic Investigations of Cooperative Catalysis in the Enantioselective Fluorination of Epoxides. J. Am. Chem. Soc. 2011, 133, 16001-16012. [CrossRef]

80. Wu, M.H.; Jacobsen, E.N. Asymmetric ring opening of meso epoxides with thiols: Enantiomeric enrichment using a bifunctional nucleophile. J. Org. Chem. 1998, 63, 5252-5254. [CrossRef]

81. Wu, J.; Hou, X.-L.; Dai, L.-X.; Xia, L.-J.; Tang, M.-H. Enantioselective ring opening of meso-epoxides with thiols catalyzed by a chiral (salen) Ti (IV) complex. Tetrahedron Asymmetry 1998, 9, 3431-3436. [CrossRef]

82. Li, Z.; Zhou, Z.; Li, K.; Wang, L.; Zhou, Q.; Tang, C. Regio-and stereoselective ring-opening of epoxides using organic dithiophosphorus acids as nucleophiles. Tetrahedron Lett. 2002, 43, 7609-7611. [CrossRef]

83. Zhou, Z.; Li, Z.; Quanyong, W.; Liu, B.; Li, K.; Zhao, G.; Zhou, Q.; Tang, C. (Salen) Ti (IV) complex catalyzed asymmetric ring-opening of epoxides using dithiophosphorus acid as the nucleophile. J. Organomet. Chem. 2006, 691, 5790-5797. [CrossRef]

84. Yang, M.; Zhu, C.; Yuan, F.; Huang, Y.; Pan, Y. Enantioselective Ring-Opening Reaction of m eso-Epoxides with ArSeH Catalyzed by Heterometallic Ti-Ga-Salen System. Org. Lett. 2005, 7, 1927-1930. [CrossRef]

85. Sun, J.; Yang, M.; Yuan, F.; Jia, X.; Yang, X.; Pan, Y.; Zhu, C. Catalytic Asymmetric Ring-Opening Reaction of meso-Epoxides with Aryl Selenols and Thiols Catalyzed by a Heterobimetallic Gallium-Titanium-Salen Complex. Adv. Synth. Catal. 2009, 351, 920-930. [CrossRef]

86. Sun, J.; Yuan, F.; Yang, M.; Pan, Y.; Zhu, C. Enantioselective ring-opening reaction of meso-epoxides with ArSH catalyzed by heterobimetallic Ti-Ga-Salen system. Tetrahedron Lett. 2009, 50, 548-551. [CrossRef]

87. Tiecco, M.; Testaferri, L.; Marini, F.; Sternativo, S.; Del Verme, F.; Santi, C.; Bagnoli, L.; Temperini, A. Synthesis of enantiomerically enriched $\beta$-hydroxy selenides by catalytic asymmetric ring opening of meso-epoxides with (phenylseleno) silanes. Tetrahedron 2008, 64, 3337-3342. [CrossRef]

88. Scheffler, U.; Mahrwald, R. Recent Advances in Organocatalytic Methods for Asymmetric C-C Bond Formation. Chem. Eur. J. 2013, 19, 14346-14396. [CrossRef] [PubMed]

89. Brahmachari, G. Design for carbon-carbon bond forming reactions under ambient conditions. RSC Adv. 2016, 6, 64676-64725. [CrossRef]

90. Bandini, M.; Cozzi, P.G.; Melchiorre, P.; Umani-Ronchi, A. Kinetic Resolution of Epoxides by a C-C Bond-Forming Reaction: Highly Enantioselective Addition of Indoles to cis, trans, and meso Aromatic Epoxides Catalyzed by [Cr (salen)] Complexes. Angew. Chem. Int. Ed. 2004, 43, 84-87. [CrossRef] [PubMed]

91. Keith, J.M.; Larrow, J.F.; Jacobsen, E.N. Practical considerations in kinetic resolution reactions. Adv. Synth. Catal. 2001, 343, 5-26. [CrossRef]

92. Larrow, J.F.; Schaus, S.E.; Jacobsen, E.N. Kinetic resolution of terminal epoxides via highly regioselective and enantioselective ring opening with TMSN3. An efficient, catalytic route to 1, 2-amino alcohols. J. Am. Chem. Soc. 1996, 118, 7420-7421. [CrossRef]

93. Schaus, S.E.; Jacobsen, E.N. Dynamic kinetic resolution of epichlorohydrin via enantioselective catalytic ring opening with TMSN3. Practical synthesis of aryl oxazolidinone antibacterial agents. Tetrahedron Lett. 1996, 37, 7937-7940. [CrossRef]

94. Lebel, H.; Jacobsen, E.N. Chromium catalyzed kinetic resolution of 2,2-disubstituted epoxides. Tetrahedron Lett. 1999, 40, 7303-7306. [CrossRef]

95. Lebel, H.; Jacobsen, E.N. Enantioselective Total Synthesis of Taurospongin A. J. Org. Chem. 1998, 63, 9624-9625. [CrossRef]

96. Dioos, B.M.L.; Jacobs, P.A. CrIII(salen) catalysed asymmetric ring opening of monocyclic terpene-epoxides. Tetrahedron Lett. 2003, 44, 4715-4717. [CrossRef]

97. Dioos, B.M.L.; Jacobs, P.A. Microwave-assisted Cr(salen)-catalysed asymmetric ring opening of epoxides. J. Catal. 2005, 235, 428-430. [CrossRef]

98. Chen, S.-W.; Thakur, S.S.; Li, W.; Shin, C.-K.; Kawthekar, R.B.; Kim, G.-J. Efficient catalytic synthesis of optically pure 1,2-azido alcohols through enantioselective epoxide ring opening with HN3. J. Mol. Catal. A Chem. 2006, 259, 116-120. [CrossRef] 
99. Bai, S.; Li, B.; Peng, J.; Zhang, X.; Yang, Q.; Li, C. Promoted activity of Cr(Salen) in a nanoreactor for kinetic resolution of terminal epoxides. Chem. Sci. 2012, 3, 2864-2867. [CrossRef]

100. Bartoli, G.; Bosco, M.; Carlone, A.; Locatelli, M.; Melchiorre, P.; Sambri, L. Asymmetric Catalytic Synthesis of Enantiopure N-Protected 1,2-Amino Alcohols. Org. Lett. 2004, 6, 3973-3975. [CrossRef]

101. Baudequin, C.; Baudoux, J.; Levillain, J.; Cahard, D.; Gaumont, A.-C.; Plaquevent, J.-C. Ionic liquids and chirality: Opportunities and challenges. Tetrahedron Asymmetry 2003, 14, 3081-3093. [CrossRef]

102. Kureshy, R.I.; Prathap, K.J.; Agrawal, S.; Kumar, M.; Khan, N.-u.H.; Abdi, S.H.R.; Bajaj, H.C. Highly Efficient Recyclable CoIII-salen Complexes in the Catalyzed Asymmetric Aminolytic Kinetic Resolution of Aryloxy/Terminal Epoxides for the Simultaneous Production of N-Protected 1,2-Amino Alcohols and the Corresponding Epoxides in High Optical Purity. Eur. J. Org. Chem. 2009, 2009, 2863-2871. [CrossRef]

103. Kureshy, R.I.; Kumar, M.; Agrawal, S.; Khan, N.-u.H.; Abdi, S.H.R.; Bajaj, H.C. Aminolytic kinetic resolution of trans epoxides for the simultaneous production of chiral trans $\beta$-amino alcohols in the presence of chiral $\mathrm{Cr}(\mathrm{III})$ salen complex using an ionic liquid as a green reaction media. Tetrahedron Asymmetry 2010, 21, 451-456. [CrossRef]

104. Kureshy, R.I.; Singh, S.; Khan, N.-u.H.; Abdi, S.H.R.; Agrawal, S.; Jasra, R.V. Enantioselective aminolytic kinetic resolution (AKR) of epoxides catalyzed by recyclable polymeric $\mathrm{Cr}(\mathrm{III})$ salen complexes. Tetrahedron Asymmetry 2006, 17, 1638-1643. [CrossRef]

105. Kureshy, R.I.; Prathap, K.J.; Singh, S.; Agrawal, S.; Khan, N.-U.H.; Abdi, S.H.R.; Jasra, R.V. Chiral recyclable dimeric and polymeric $\mathrm{Cr}$ (III) salen complexes catalyzed aminolytic kinetic resolution of trans-aromatic epoxides under microwave irradiation. Chirality 2007, 19, 809-815. [CrossRef] [PubMed]

106. Kureshy, R.I.; Prathap, K.J.; Roy, T.; Maity, N.C.; Khan, N.-u.H.; Abdi, S.H.R.; Bajaj, H.C. Reusable Chiral Dicationic Chromium(III) Salen Catalysts for Aminolytic Kinetic Resolution of trans-Epoxides. Adv. Synth. Catal. 2010, 352, 3053-3060. [CrossRef]

107. Tak, R.; Kumar, M.; Menapara, T.; Choudhary, M.K.; Kureshy, R.I.; Khan, N.-u.H. Asymmetric Catalytic Syntheses of Pharmaceutically Important $\beta$-Amino- $\alpha$-Hydroxyl Esters by Enantioselective Aminolysis of Methyl Phenylglycidate. ChemCatChem 2017, 9, 322-328. [CrossRef]

108. Kumar, M.; Kureshy, R.I.; Shah, A.K.; Das, A.; Khan, N.-u.H.; Abdi, S.H.R.; Bajaj, H.C. Asymmetric Aminolytic Kinetic Resolution of Racemic Epoxides Using Recyclable Chiral Polymeric Co(III)-Salen Complexes: A Protocol for Total Utilization of Racemic Epoxide in the Synthesis of (R)-Naftopidil and (S)-Propranolol. J. Org. Chem. 2013, 78, 9076-9084. [CrossRef]

109. Tokunaga, M.; Larrow, J.F.; Kakiuchi, F.; Jacobsen, E.N. Asymmetric Catalysis with Water: Efficient Kinetic Resolution of Terminal Epoxides by Means of Catalytic Hydrolysis. Science 1997, 277, 936-938. [CrossRef]

110. Schaus, S.E.; Brandes, B.D.; Larrow, J.F.; Tokunaga, M.; Hansen, K.B.; Gould, A.E.; Furrow, M.E.; Jacobsen, E.N. Highly Selective Hydrolytic Kinetic Resolution of Terminal Epoxides Catalyzed by Chiral (salen)CoIII Complexes. Practical Synthesis of Enantioenriched Terminal Epoxides and 1,2-Diols. J. Am. Chem. Soc. 2002, 124, 1307-1315. [CrossRef]

111. Kureshy, R.I.; Khan, N.H.; Abdi, S.H.R.; Patel, S.T.; Jasra, R.V. Simultaneous production of chirally enriched epoxides and 1,2-diols from racemic epoxides via hydrolytic kinetic resolution (HKR). J. Mol. Catal. A Chem. 2002, 179, 73-77. [CrossRef]

112. Kureshy, R.I.; Singh, S.; Khan, N.U.H.; Abdi, S.H.R.; Ahmad, I.; Bhatt, A.; Jasra, R.V. Improved catalytic activity of homochiral dimeric cobalt-salen complex in hydrolytic kinetic resolution of terminal racemic epoxides. Chirality 2005, 17, 590-594. [CrossRef]

113. Venkatasubbaiah, K.; Gill, C.S.; Takatani, T.; Sherrill, C.D.; Jones, C.W. A Versatile Co(bisalen) Unit for Homogeneous and Heterogeneous Cooperative Catalysis in the Hydrolytic Kinetic Resolution of Epoxides. Chem. Eur. J. 2009, 15, 3951-3955. [CrossRef]

114. Wezenberg, S.J.; Kleij, A.W. Cooperative Activation in the Hydrolytic Kinetic Resolution of Epoxides by a Bis-Cobalt(III)salen-Calix 4 arene Hybrid. Adv. Synth. Catal. 2010, 352, 85-91. [CrossRef]

115. Ready, J.M.; Jacobsen, E.N. A practical oligomeric (salen)Co catalyst for asymmetric epoxide ring-opening reactions. Angew. Chem. Int. Ed. 2002, 41, 1374-1377. [CrossRef]

116. Kahn, M.G.C.; Weck, M. Highly crosslinked polycyclooctyl-salen cobalt (III) for the hydrolytic kinetic resolution of terminal epoxides. Catal. Sci. Technol. 2012, 2, 386-389. [CrossRef] 
117. Sadhukhan, A.; Khan, N.-u.H.; Roy, T.; Kureshy, R.I.; Abdi, S.H.R.; Bajaj, H.C. Asymmetric Hydrolytic Kinetic Resolution with Recyclable Macrocyclic CoIII-Salen Complexes: A Practical Strategy in the Preparation of (R)-Mexiletine and (S)-Propranolol. Chem. Eur. J. 2012, 18, 5256-5260. [CrossRef]

118. Roy, T.; Barik, S.; Kumar, M.; Kureshy, R.I.; Ganguly, B.; Khan, N.-u.H.; Abdi, S.H.R.; Bajaj, H.C. Asymmetric hydrolytic kinetic resolution with recyclable polymeric Co(III)-salen complexes: A practical strategy in the preparation of (S)-metoprolol, (S)-toliprolol and (S)-alprenolol: Computational rationale for enantioselectivity. Catal. Sci. Technol. 2014, 4, 3899-3908. [CrossRef]

119. Hong, X.; Mellah, M.; Bordier, F.; Guillot, R.; Schulz, E. Electrogenerated Polymers as Efficient and Robust Heterogeneous Catalysts for the Hydrolytic Kinetic Resolution of Terminal Epoxides. ChemCatChem 2012, 4, 1115-1121. [CrossRef]

120. Dandachi, H.; Nasrallah, H.; Ibrahim, F.; Hong, X.; Mellah, M.; Jaber, N.; Schulz, E. Chiral calix-salen cobalt complexes, catalysts for the enantioselective dynamic hydrolytic kinetic resolution of epibromohydrin. J. Mol. Catal. A Chem. 2014, 395, 457-462. [CrossRef]

121. Breinbauer, R.; Jacobsen, E.N. Cooperative Asymmetric Catalysis with Dendrimeric [Co(salen)] Complexes. Angew. Chem. Int. Ed. 2000, 39, 3604-3607. [CrossRef]

122. Song, Y.; Yao, X.; Chen, H.; Bai, C.; Hu, X.; Zheng, Z. Highly enantioselective resolution of terminal epoxides using polymeric catalysts. Tetrahedron Lett. 2002, 43, 6625-6627. [CrossRef]

123. Song, Y.M.; Chen, H.L.; Hu, X.Q.; Bai, C.M.; Zheng, Z. Highly enantioselective resolution of terminal epoxides with cross-linked polymeric salen-Co(III) complexes. Tetrahedron Lett. 2003, 44, 7081-7085. [CrossRef]

124. Zheng, X.; Jones, C.W.; Weck, M. Poly(styrene)-Supported Co-Salen Complexes as Efficient Recyclable Catalysts for the Hydrolytic Kinetic Resolution of Epichlorohydrin. Chem. Eur. J. 2006, 12, 576-583. [CrossRef]

125. Zheng, X.; Jones, C.W.; Weck, M. Engineering Polymer-Enhanced Bimetallic Cooperative Interactions in the Hydrolytic Kinetic Resolution of Epoxides. Adv. Synth. Catal. 2008, 350, 255-261. [CrossRef]

126. Rossbach, B.M.; Leopold, K.; Weberskirch, R. Self-Assembled Nanoreactors as Highly Active Catalysts in the Hydrolytic Kinetic Resolution (HKR) of Epoxides in Water. Angew. Chem. Int. Ed. 2006, 45, 1309-1312. [CrossRef] [PubMed]

127. Thakur, S.S.; Li, W.J.; Kim, S.J.; Kim, G.J. Highly reactive and enantioselective kinetic resolution of terminal epoxides with $\mathrm{H}_{2} \mathrm{O}$ and $\mathrm{HCl}$ catalyzed by new chiral (salen)Co complex linked with Al. Tetrahedron Lett. 2005, 46, 2263-2266. [CrossRef]

128. Thakur, S.S.; Chen, S.W.; Li, W.J.; Shin, C.K.; Kim, S.J.; Koo, Y.M.; Kim, G.J. A new dinuclear chiral salen complexes for asymmetric ring opening and closing reactions: Synthesis of valuable chiral intermediates. J. Organomet. Chem. 2006, 691, 1862-1872. [CrossRef]

129. Jiang, C.J.; Chen, Z.R. Chiral cobalt salen complexes containing Lewis acid: A highly reactive and enantioselective catalyst for the hydrolytic kinetic resolution of terminal epoxides. Kinet. Catal. 2008, 49, 474-478. [CrossRef]

130. Kawthekar, R.B.; Bi, W.-t.; Kim, G.-J. Asymmetric ring opening of epoxides catalyzed by novel heterobimetallic Schiff-bases containing transition metal salts. Bull. Korean Chem. Soc. 2008, 29, 313-318. [CrossRef]

131. Jiang, C. Asymmetric ring opening of terminal epoxides via kinetic resolution catalyzed by chiral (salen)Co mixture. Kinet. Catal. 2011, 52, 691-696. [CrossRef]

132. Park, J.; Lang, K.; Abboud, K.A.; Hong, S. Self-Assembly Approach toward Chiral Bimetallic Catalysts: Bis-Urea-Functionalized (Salen)Cobalt Complexes for the Hydrolytic Kinetic Resolution of Epoxides. Chem. Eur. J. 2011, 17, 2236-2245. [CrossRef]

133. Yang, H.; Zhang, L.; Su, W.; Yang, Q.; Li, C. Asymmetric ring-opening of epoxides on chiral Co(Salen) catalyst synthesized in SBA-16 through the "ship in a bottle" strategy. J. Catal. 2007, 248, 204-212. [CrossRef]

134. Yang, H.; Zhang, L.; Zhong, L.; Yang, Q.; Li, C. Enhanced Cooperative Activation Effect in the Hydrolytic Kinetic Resolution of Epoxides on [Co(salen)] Catalysts Confined in Nanocages. Angew. Chem. Int. Ed. 2007, 46, 6861-6865. [CrossRef]

135. Cavazzini, M.; Quici, S.; Pozzi, G. Hydrolytic kinetic resolution of terminal epoxides catalyzed by fluorous chiral Co(salen) complexes. Tetrahedron 2002, 58, 3943-3949. [CrossRef]

136. Shepperson, I.; Cavazzini, M.; Pozzi, G.; Quici, S. Fluorous biphasic hydrolytic kinetic resolution of terminal epoxides. J. Fluorine Chem. 2004, 125, 175-180. [CrossRef] 
137. Rim Oh, C.; Joon Choo, D.; Ho Shim, W.; Hoon Lee, D.; Joo Roh, E.; Lee, S.-g.; Eui Song, C. Chiral $\mathrm{Co}(\mathrm{iii})($ salen)-catalysed hydrolytic kinetic resolution of racemic epoxides in ionic liquids. Chem. Commun. 2003, 1100-1101. [CrossRef] [PubMed]

138. Aerts, S.; Weyten, H.; Buekenhoudt, A.; Gevers, L.E.M.; Vankelecom, I.F.J.; Jacobs, P.A. Recycling of the homogeneous Co-Jacobsen catalyst through solvent-resistent nanofiltration (SRNF). Chem. Commun. 2004, 710-711. [CrossRef]

139. Annis, D.A.; Jacobsen, E.N. Polymer-supported chiral Co(salen) complexes: Synthetic applications and mechanistic investigations in the hydrolytic kinetic resolution of terminal epoxides. J. Am. Chem. Soc. 1999, 121, 4147-4154. [CrossRef]

140. Solodenko, W.; Jas, G.; Kunz, U.; Kirschning, A. Continuous enantioselective kinetic resolution of terminal epoxides using immobilized chiral cobalt-salen complexes. Synthesis 2007, 583-589. [CrossRef]

141. Kunz, U.; Schönfeld, H.; Solodenko, W.; Jas, G.; Kirschning, A. Manufacturing and Construction of PASSflow Flow Reactors and Their Utilization in Suzuki-Miyaura Cross-Coupling Reactions. Ind. Eng. Chem. Res. 2005, 44, 8458-8467. [CrossRef]

142. Goyal, P.; Zheng, X.; Weck, M. Enhanced Cooperativity in Hydrolytic Kinetic Resolution of Epoxides using Poly(styrene) Resin-Supported Dendronized Co-(Salen) Catalysts. Adv. Synth. Catal. 2008, 350, 1816-1822. [CrossRef]

143. Kahn, M.G.C.; Stenlid, J.H.; Weck, M. Poly(styrene) Resin-Supported Cobalt(III) Salen Cyclic Oligomers as Active Heterogeneous HKR Catalysts. Adv. Synth. Catal. 2012, 354, 3016-3024. [CrossRef]

144. Gill, C.S.; Venkatasubbaiah, K.; Phan, N.T.S.; Weck, M.; Jones, C.W. Enhanced Cooperativity through Design: Pendant CoIII-Salen Polymer Brush Catalysts for the Hydrolytic Kinetic Resolution of Epichlorohydrin (Salen=N,N'-Bis(salicylidene)ethylenediamine Dianion). Chem. Eur. J. 2008, 14, 7306-7313. [CrossRef]

145. Belser, T.; Jacobsen, E.N. Cooperative catalysis in the hydrolytic kinetic resolution of epoxides by chiral (salen)Co(III) complexes immobilized on gold colloids. Adv. Synth. Catal. 2008, 350, 967-971. [CrossRef]

146. Kawthekar, R.B.; Lee, Y.-H.; Kim, G.-J. Asymmetric catalysis by chiral salen complexes immobilized on mesoporous materials having modified pore channel system by dimethylcarbonate. J. Porous Mater. 2009, 16, 367-378. [CrossRef]

147. Kim, Y.-S.; Guo, X.-F.; Kim, G.-J. Highly active new chiral Co(III) salen catalysts immobilized by electrostatic interaction with sulfonic acid linkages on ordered mesoporous SBA-16 silica. Chem. Commun. 2009, 4296-4298. [CrossRef] [PubMed]

148. Kim, Y.-S.; Lee, C.-Y.; Kim, G.-J. Asymmetric Ring Opening of Terminal Epoxides Catalyzed by Chiral Co(III)-BF3 Salen Complex Immobilized on SBA-16. Bull. Korean Chem. Soc. 2009, 30, 1771-1777. [CrossRef]

149. Kim, Y.-S.; Lee, C.-Y.; Kim, G.-J. Synthesis of New Bimetallic Chiral Salen Catalyst Bearing Co(BF4)(2) Salt and Its Application in Asymmetric Ring Opening of Epoxide. Bull. Korean Chem. Soc. 2010, 31, 2973-2979. [CrossRef]

150. Kim, G.-J.; Lee, H.; Kim, S.-J. Catalytic activity and recyclability of new enantioselective chiral Co-salen complexes in the hydrolytic kinetic resolution of epichlorohydrine. Tetrahedron Lett. 2003, 44, 5005-5008. [CrossRef]

151. Nielsen, L.P.C.; Stevenson, C.P.; Blackmond, D.G.; Jacobsen, E.N. Mechanistic investigation leads to a synthetic improvement in the hydrolytic kinetic resolution of terminal epoxides. J. Am. Chem. Soc. 2004, 126, 1360-1362. [CrossRef] [PubMed]

152. Sun, K.; Li, W.-X.; Feng, Z.; Li, C. Cooperative activation in ring-opening hydrolysis of epoxides by Co-salen complexes: A first principle study. Chem. Phys. Lett. 2009, 470, 259-263. [CrossRef]

153. Kennedy, M.R.; Burns, L.A.; Sherrill, C.D. Counterion and Substrate Effects on Barrier Heights of the Hydrolytic Kinetic Resolution of Terminal Epoxides Catalyzed by Co(III)-salen. J. Phys. Chem. A 2015, 119, 403-409. [CrossRef]

154. Jain, S.; Zheng, X.; Jones, C.W.; Weck, M.; Davis, R.J. Importance of counterion reactivity on the deactivation of co-salen catalysts in the hydrolytic kinetic resolution of epichlorohydrin. Inorg. Chem. 2007, 46, 8887-8896. [CrossRef]

155. Jain, S.; Venkatasubbaiah, K.; Jones, C.W.; Davis, R.J. Factors influencing recyclability of Co(III)-salen catalysts in the hydrolytic kinetic resolution of epichlorohydrin. J. Mol. Catal. A Chem. 2010, 316, 8-15. [CrossRef] 
156. Ford, D.D.; Nielsen, L.P.C.; Zuend, S.J.; Musgrave, C.B.; Jacobsen, E.N. Mechanistic Basis for High Stereoselectivity and Broad Substrate Scope in the (salen)Co(III)-Catalyzed Hydrolytic Kinetic Resolution. J. Am. Chem. Soc. 2013, 135, 15595-15608. [CrossRef] [PubMed]

157. Berkessel, A.; Ertuerk, E. Hydrolytic kinetic resolution of epoxides catalyzed by chromium(III)-endo,endo-2,5diaminonorbornane-salen Cr(III)-DIANANE-salen complexes. Improved activity, low catalyst loading. Adv. Synth. Catal. 2006, 348, 2619-2625. [CrossRef]

158. Lee, Y.; Yu, J.; Park, K.; Kim, G.J. Superior Effect of Ultrasonic Homogenization to Mechanical Agitation on Accelerating Reaction Rates in Asymmetric Ring Opening of Epoxides. Bull. Korean Chem. Soc. 2017, 38, 795-803. [CrossRef]

159. Wright, J.L.; Gregory, T.F.; Heffner, T.G.; MacKenzie, R.G.; Pugsley, T.A.; Meulen, S.V.; Wise, L.D. Discovery of selective dopamine D4 receptor antagonists: 1-Aryloxy-3-(4-aryloxypiperidinyl)-2-propanols. Bioorgan. Med. Chem. Lett. 1997, 7, 1377-1380. [CrossRef]

160. Ready, J.M.; Jacobsen, E.N. Asymmetric Catalytic Synthesis of $\alpha$-Aryloxy Alcohols: Kinetic Resolution of Terminal Epoxides via Highly Enantioselective Ring-Opening with Phenols. J. Am. Chem. Soc. 1999, 121, 6086-6087. [CrossRef]

161. Peukert, S.; Jacobsen, E.N. Enantioselective Parallel Synthesis Using Polymer-Supported Chiral Co(salen) Complexes. Org. Lett. 1999, 1, 1245-1248. [CrossRef]

162. Zhu, X.; Venkatasubbaiah, K.; Weck, M.; Jones, C.W. Highly active oligomeric Co(salen) catalysts for the asymmetric synthesis of alpha-aryloxy or alpha-alkoxy alcohols via kinetic resolution of terminal epoxides. J. Mol. Catal. A Chem. 2010, 329, 1-6. [CrossRef]

163. Kawthekar, R.B.; Ahn, C.-H.; Kim, G.-J. Kinetic resolution of terminal epoxides with phenols promoted by heterometallic Co-Al and Co-Ga salen complexes. Catal. Lett. 2007, 115, 62-69. [CrossRef]

164. Lee, K.-Y.; Lee, C.-Y.; Kim, G.-J. Asymmetric synthesis of alpha-aryloxy alcohols via kinetic resolution of terminal epoxides catalyzed by (R,R)-N,N'-bis(2-hydroxy-5-tert-butylsalisilidine)-1,2-cyclohexenediamino cobalt. React. Kinet. Catal. Lett. 2008, 93, 75-83. [CrossRef]

165. Guo, X.-F.; Kim, Y.-S.; Kim, G.-J. Chiral (Salen) Cobalt Complexes Encapsulated in Mesoporous MFI as an Enantioselective Catalyst for Asymmetric Ring Opening of Terminal Epoxides. Top. Catal. 2009, 52, 153-160. [CrossRef]

166. Lee, K.-Y.; Lee, C.-Y.; Kim, G.-J. Non-Covalent Immobilization of Chiral (Salen) Complexes on HF-treated Mesoporous MFI-type Zeolite for Asymmetric Catalysis. Bull. Korean Chem. Soc. 2009, 30, 389-396. [CrossRef]

167. Kim, Y.-S.; Guo, X.-f.; Kim, G.-J. Asymmetric Ring Opening Reaction of Catalyst Immobilized on Silica Monolith with Bimodal Meso/Macroscopic Pore Structure. Top. Catal. 2009, 52, 197-204. [CrossRef]

168. Jeon, H.-G.; Choi, S.D.; Jeon, S.K.; Park, G.W.; Yang, J.Y.; Kim, G.-J. Application of Mesoporous Silica Foam for Immobilization of Salen Complexes in Chiral Intermediates Synthesis. Bull. Korean Chem. Soc. 2015, 36, 1396-1404. [CrossRef]

169. Li, W.J.; Thakur, S.S.; Chen, S.W.; Shin, C.K.; Kawthekar, R.B.; Kim, G.J. Synthesis of optically active 2-hydroxy monoesters via-kinetic resolution and asymmetric cyclization catalyzed by heterometallic chiral (salen) Co complex. Tetrahedron Lett. 2006, 47, 3453-3457. [CrossRef]

170. Lee, Y.W.; Yang, H.C.; Kim, G.-J. Synthesis of Highly Enantiomerically Enriched Arenesulfonic Acid 2-Hydroxy Esters via Kinetic Resolution of Terminal Epoxides. Appl. Chem. Eng. 2016, 27, 490-494. [CrossRef]

171. Hajra, S.; Roy, S. Feedback Inhibition in Chemical Catalysis Leads the Dynamic Kinetic to Kinetic Resolution in C3-Indolylation of Spiro-epoxyoxindoles. Org. Lett. 2020, 22, 1458-1463. [CrossRef]

172. Hajra, S.; Maity, S.; Maity, R. Efficient Synthesis of 3,3'-Mixed Bisindoles via Lewis Acid Catalyzed Reaction of Spiro-epoxyoxindoles and Indoles. Org. Lett. 2015, 17, 3430-3433. [CrossRef]

173. Linker, T. The Jacobsen-Katsuki Epoxidation and Its Controversial Mechanism. Angew. Chem. Int. Ed. 1997, 36, 2060-2062. [CrossRef]

(C) 2020 by the authors. Licensee MDPI, Basel, Switzerland. This article is an open access article distributed under the terms and conditions of the Creative Commons Attribution (CC BY) license (http://creativecommons.org/licenses/by/4.0/). 